Physica Scripta. Vol. 38, 347-369, 1988.

\title{
Spectrum of Doubly lonized Xenon (Xe III)
}

\author{
W. Persson and C.-G. Wahlström \\ Department of Physics, Lund Institute of Technology, Box 118, S-221 00 Lund, Sweden \\ G. Bertucoelli and H. O. Di Rocco \\ Departamento de Fisica, Facultad de Ciencias Exactas, Pinto 399, 7000 Tandil, Argentina \\ and
}

J. G. Reyna Almandos and M. Gallardo

Centro de Investigaciones Opticas, Casilla de Correo 124, 1900 La Plata, Argentina

Received September. IS. 1987: accepted November 12. 1987

\begin{abstract}
The spectrum of doubly ionized xenon has been investigated. The study is based on photographic recordings of xenon spectra in the $490-8900 \AA$ range. The number of classified lines has been increased from about 300 to about 1400. The lines have been classified as transitions between 73 even levels belonging to the $5 s^{2} 5 p^{4}, 5 s^{2} 5 p^{3} 6 p, 4 f, 5 f$ and $5 s^{6} 5 p^{6}$ configurations, and 83 odd levels belonging to the $5 s 5 p^{5}, 5 s^{2} 5 p^{3} 6 s, 7 s, 5 d$ and $6 d$ configurations. In particular, the classifications include most of the Xe III laser lines. The experimentally observed level structures are compared with the results of Hartree-Fock calculations and least-squares fits. A comparison is also made between the results of the present analysis and the published data on the $\mathrm{Xe} \mathrm{N}_{\&} \mathrm{OO}$ Auger spectrum.
\end{abstract}

\section{Introduction}

The doubly ionized xenon atom, $\mathrm{Xe}^{2+}(Z=54)$, is isoelectronic with neutral tellurium. The ground configuration in this sequence is $5 s^{2} 5 p^{4}$. Although there has been a great demand, e.g., from laser and collision physics, for improved data on the Xe III spectrum and energy level system for many years, very little work has been reported since the 1930's when Boyce [1], Humphreys [2] and Humphreys et al. [3] undertook extensive studies of the spectrum. A few reports have appeared treating the lower levels of the spectrum [4-6] and the $5 s^{0} 5 p^{6}{ }^{1} S_{0}$ level $[7,8]$.

A large number of strong xenon laser lines were reported some 20 years ago [9]. Primarily due to the work of the group in La Plata, the laser lines were classified as originating in doubly and trebly ionized xenon, but no further classifications were possible due to the lack of relevant spectroscopic data.

In the present investigation we have recorded xenon spectra photographically in the 490-6900 $\AA$ range. When analysing the vast amount of experimental data we have made extensive use of Hartree-Fock calculations and parametric fits. Configuration-interaction ( $\mathrm{Cl}$ ) effects, including Rydberg series $\mathbf{C I}$, have been included in the calculations. The configurations studied are $5 s^{2} 5 p^{4}, 5 s 5 p^{5}, 5 s^{0} 5 p^{6}, 5 s^{2} 5 p^{3} 6 p$, $6 s, 7 s, 5 d, 6 d$ and $4 f$. The lowest term of the $5 f$ configuration has also been located. The number of classified lines has been increased from about 300 to, in all, 1400. These lines originate from transitions between 73 even- and 83 odd-parity levels. As a consequence of the present analysis it has been possible to classify the majority of the laser lines ascribed to Xe III.

The extended analysis of the Xe III spectrum also has some consequences for the interpretation of the Auger spectrum following ionization in the $4 d$ subshell of neutral xenon.

\section{Experimental}

The vacuum-ultraviolet part of the spectrum was recorded in Lund. Two different light sources were used: a direct-current hollow-cathode discharge [10] and a theta-pinch discharge [11]. The hollow-cathode source gives a Xe III spectrum of better quality as regards resolution and obtainabie wavelength accuracy, while the theta-pinch exposures were of great value in the determination of the ionization stages of the observed lines. The spectrum was photographed on a $3 \mathrm{~m}$ normal-incidence spectrograph with a plate factor of $2.77 \AA / \mathrm{mm}$ in the first diffraction order [12]. The wavelength range above $2000 \AA$ was recorded on a $3.4 \mathrm{~m}$ Ebert planegrating spectrograph in La Plata. This instrument has a plate factor of $5 \AA / \mathrm{mm}$ in the first diffraction order. The results of the wavelength measurements in air have been discussed previously [13]. The spectrum was excited in a laser-tube-like source (without end-mirrors) about $1 \mathrm{~m}$ in length and with an inner diameter of $3 \mathrm{~mm}$. The tube has inner electrodes and was viewed end-on [14].

The wavelengths and intensities of all classified Xe III lines are given in Table I. In the long-wavelength end of the spectrum, outside the range covered by the present recording, a few lines have been included from an unpublished xenon line-list by Humphreys [15]. The quality of the recorded spectra does not permit very accurate wavelength determination. Most lines are fairly wide. The overall wavelength accuracy is estimated to be $0.05 A$ in the air region and $0.02 \AA$ in the vacuum-ultraviolet wavelength region.

The intensity figures are visual estimates of photographic density, and are on a uniform scale only within limited wavelength ranges. For the lines quoted from Humphreys' list the intensities are on a completely different scale.

All the experimentally established Xe III levels are given in Tables II and.III. The level values were determined by a least-squares procedure in which the appropriately weighted wave numbers of the identified lines were used as input. All level designations are in $\mathrm{LS}$ notation. In most cases the names given to the levels were taken from least-squares fits of the theoretical energy expressions to the experimentally observed level values. In general, the calculated purities of the states 


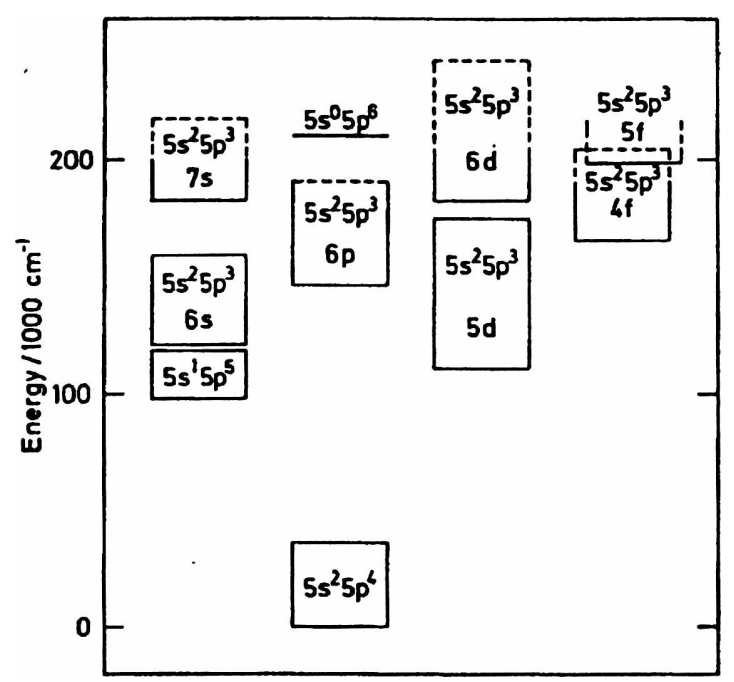

Fig. I. Gross structure of the observed Xe IIl configurations. Broken lines indicate that not all levels of the configuration have been located.

(Tables II and III) are low, showing that the coupling conditions in the configurations investigated are intermediate.

\section{Analysis}

When performing the analysis of the experimental data we were guided by theoretical predictions of the level structures. Such predictions were obtained by diagonalization of the appropriate energy matrices, including CI matrix elements. The radial parts of the matrix elements were determined in Hartree-Fock calculations. Approximate scaling factors were determined from comparisons with calculations for similar structures. Figure 1 shows the relative positions and extensions of the configurations studied. The levels in $5 s^{2} 5 p^{4}$, $5 s 5 p^{5}$ and $5 s^{0} 5 p^{6}$ were known from earlier investigations, though the designation of one level, $5 s 5 p^{5}{ }^{1} P_{1}$ has been revised. The $5 s^{2} 5 p^{3} n l$ configurations can be considered as being built on the ground configuration of Xe IV, $5 s^{2} 5 p^{3}$, with the addition of an outer electron. The parent configuration gives three terms, namely ${ }^{4} S,{ }^{2} D$ and ${ }^{2} P$. Almost all levels of the $5 s^{2} 5 p^{3} 6 p$, $6 s$ and $5 d$ configurations have been experimentally established or verified in this work. In the $4 f$ configuration, five of the levels based on the ${ }^{2} P$ parent term are missing and in the $5 s^{2} 5 p^{3} 7 s$ and $6 d$ configurations only levels based on the ${ }^{4} S$ and ${ }^{2} D$ parent terms have been located. In the $5 f$ configuration, only the levels belonging to the lowest term, $\left({ }^{4} S\right)^{5} F$, have been located with certainty.

Figure 1 shows that there is severe overlapping of configurations of the same parity. This leads to heavy mixing of states belonging to different configurations, even if the matrix elements connecting the states are small. Such mixing occurs between $6 s$ and $5 d, 7 s$ and $6 d$ and between $6 p$ and $4 f$ states.

\subsection{Even configurations}

When interpreting the observed energy-level structure of the even-parity configurations, the total energy matrix for the $5 s^{2} 5 p^{4}+5 s^{2} 5 p^{3}(6 p+4 f+5 f)+5 s 5 p^{4} 5 d+5 s^{0} 5 p^{6}$ configurations was diagonalized. The calculated energy-level values were fitted to the observed ones by least-squares fits in which some of the energy parameters were treated as free parameters (Tables IV and V).
As is evident from Fig. 1, there are large energy separations between the levels of the ground configuration and the excited configurations. In cases like this, it is customary to diagonalize the energy matrix and to perform a least-squares fit for the ground configuration separately. However, it was found that a least-squares fit to the levels of the ground configuration, omitting the effective configuration-interaction parameter $\alpha$, gives a large discrepancy between the observed and the calculated positions of the $5 s^{2} 5 p^{4} D_{2}$ level. The radial integral in the CI matrix element between the $s^{2} p^{4}$ and $s^{0} p^{6}$ configurations is very large $\left(\sim 67000 \mathrm{~cm}^{-1}\right)$. A simple perturbation calculation indicates that this interaction gives rise to a large shift $\left(\sim 8000 \mathrm{~cm}^{-1}\right)$ of the ' $S_{0}$ level of the ground configuration. In a similar way, it was found that the interaction between the ground configuration and a "pure" $5 s 5 p^{4} 5 d$ configuration gives rise to large shifts $\left(\sim 4000 \mathrm{~cm}^{-1}\right)$ of the ${ }^{3} P$ and ' $D$ levels, but not to the ' $S_{0}$ level. Evidently, large specific level shifts may occur from these interactions between distant configurations. It was also found that the $5 s^{0} 5 p^{6}{ }^{1} S_{0}$ state interacts strongly with the ' $S_{0}$ state of the $5 s 5 p^{4} 5 d$ configuration and a substantial mixing of these two states occurs.

In the light of the above discussion we decided to include the ground configuration and the high-lying $5 s 5 p^{4} 5 d$ configuration in the energy matrix of the even configurations. CI effects between all the configurations were taken into account. In particular, it was found that the large specific deviation of the $p^{4} D_{2}$ level was removed in this way, even with the configuration interaction parameters fixed at their HF values. As none of the levels belonging to the $5 s 5 p^{4} 5 d$ configuration has been established experimentally, the energy parameters of this configuration were held fixed at their HF values during the fitting process (except the $F^{2}(5 p, 5 p)$ integral which was scaled to 0.8 times the $H F$ value.)

The level structure of the $5 s^{2} 5 p^{3} 6 p$ and $4 f$ configurations is given in Fig. 2. The positions of the observed levels of the lowest term of the $5 f$ configuration are also indicated. It turns out that $4 f$ is almost as low a configuration as $6 p$. This reflects the fact that Xe III is close to the lanthanides and $4 f$ is no longer hydrogenic. All levels are given in LS notation. Generally the designations given represent the largest contribution to the eigenvector. However, for many levels the purities are very low, the largest component amounting to only about $30 \%$ in some cases. In one case we have used the second largest eigenvector component to name the level. Thus the $L S$ designations often have very little physical significance.

\subsection{Odd configurations}

The odd-parity configurations were also interpreted by means of energy matrix diagonalizations and parametric least-squares fits to the energy levels. The energy matrix included the $5 s 5 p^{3}+5 s^{2} 5 p^{3}(6 s+7 s+5 d+6 d)$ configurations (Tables VI and VII).

The detailed structure of the $5 s 5 p^{5}$ and the $5 s^{2} 5 p^{3}(5 d+6 s)$ configurations is shown in Fig. 3. The experimentally established part of the $5 s^{2} 5 p^{3}(6 d+7 s)$ configurations is shown in Fig. 4. As can be seen from the figures, there are a number of fortuitous coincidences between $6 s$ and $5 d$ levels, and between $7 s$ and $6 d$ levels causing severe mixing of the corresponding states.

All levels are given in $L S$ notation, but, as for the evenparity levels, the designations often have very little physical significance because of the severe mixing of states with 


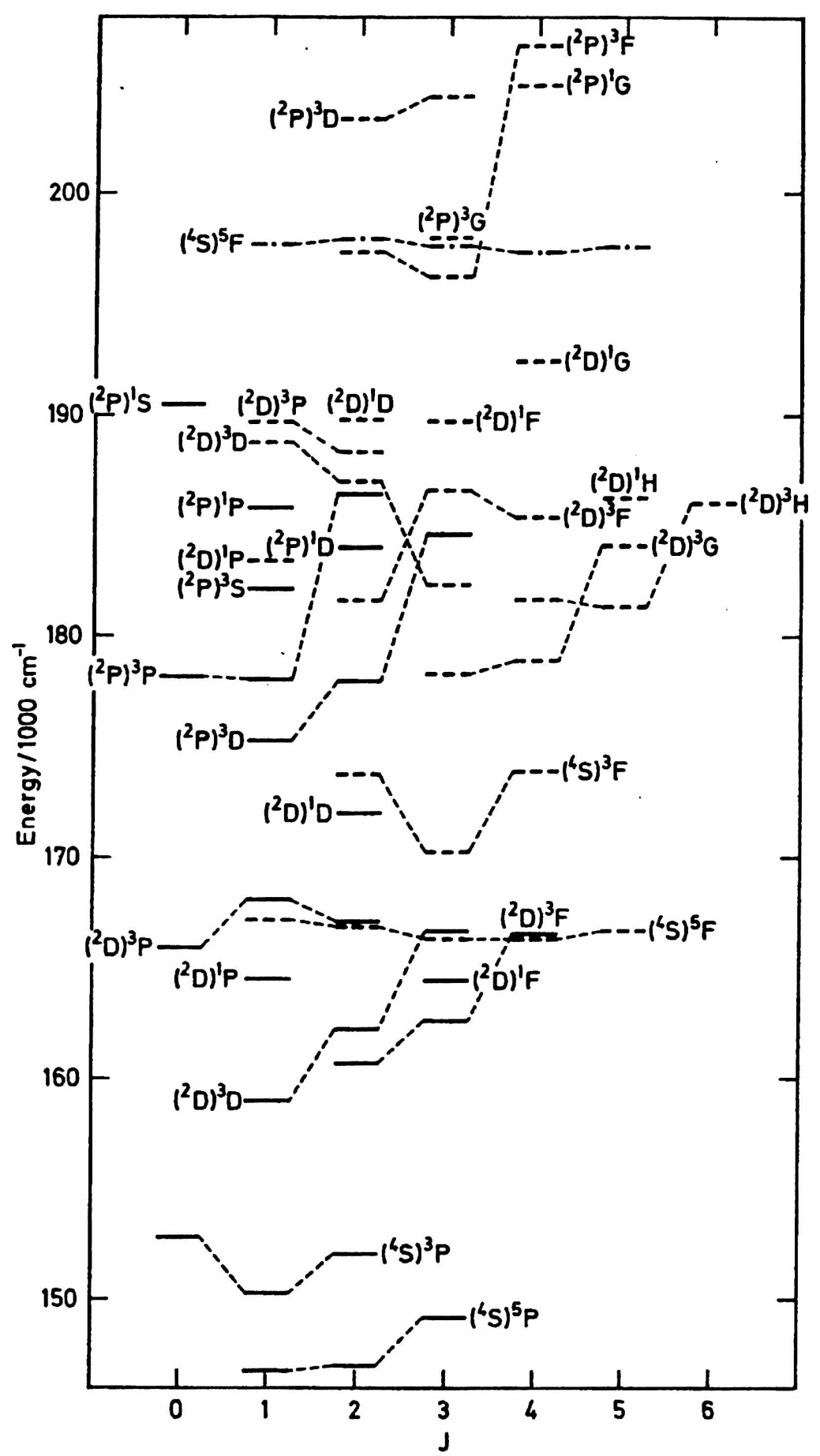

Fig. 2. Structure of the $5 s^{2} 5 p^{2}(6 p+4 f)$ configurations of Xe III. The position of the lowest $5 f$ term is also indicated. $6 p$ levels are indicated by fully drawn lines, $4 /$ levels by dashed and 5 flevels by broken lines with dots in the centre. All levels are given in the $L S$ coupling scheme. different $L$ and $S$ values. To avoid duplication of labels it has sometimes been necessary to use the second largest or even the third largest eigenvector component to name the level.

There is also strong mixing between $5 s 5 p^{5}$ and $5 s^{2} 5 p^{3} 5 d$ states. Primarily this mixing is not caused by close level coincidences, but rather by large matrix elements connecting the states. The mixing is most pronounced for the singlets. In fact, there is no level having $5 s 5 p^{5}{ }^{1} P$ as its largest eigenvector component. On the other hand, there are five levels having a substantial $5 s 5 p^{5}$ ' $P$ contribution to their eigenvectors. As will be discussed below, this mixing has some consequences for the Auger spectrum following ionization of an inner $4 d$ electron.

A general observation regarding $p^{3} d$ configurations seems to be that the ${ }^{3} S$ term of the lowest $d$ configuration is predicted far below its observed position. In the $4 p^{3} 4 d$ configuration of $\mathrm{Kr}$ III [16], Rb IV [17], Sr V [18], and Y VI [19] the discrepancy is of the order of $3000 \mathrm{~cm}^{-1}$. The discrepancy is also present in lighter elements, for instance in the $2 p^{3} 3 d$ configuration of Ne III [20]. It was shown in Refs [17] and [18] that, to a large extent, the discrepancy in the $4 p^{3} 4 d$ configurations of $\mathrm{Sr}$ V and $\mathrm{Rb}$ IV could be accounted for by the introduction of Rydberg-series configuration interactions, in 


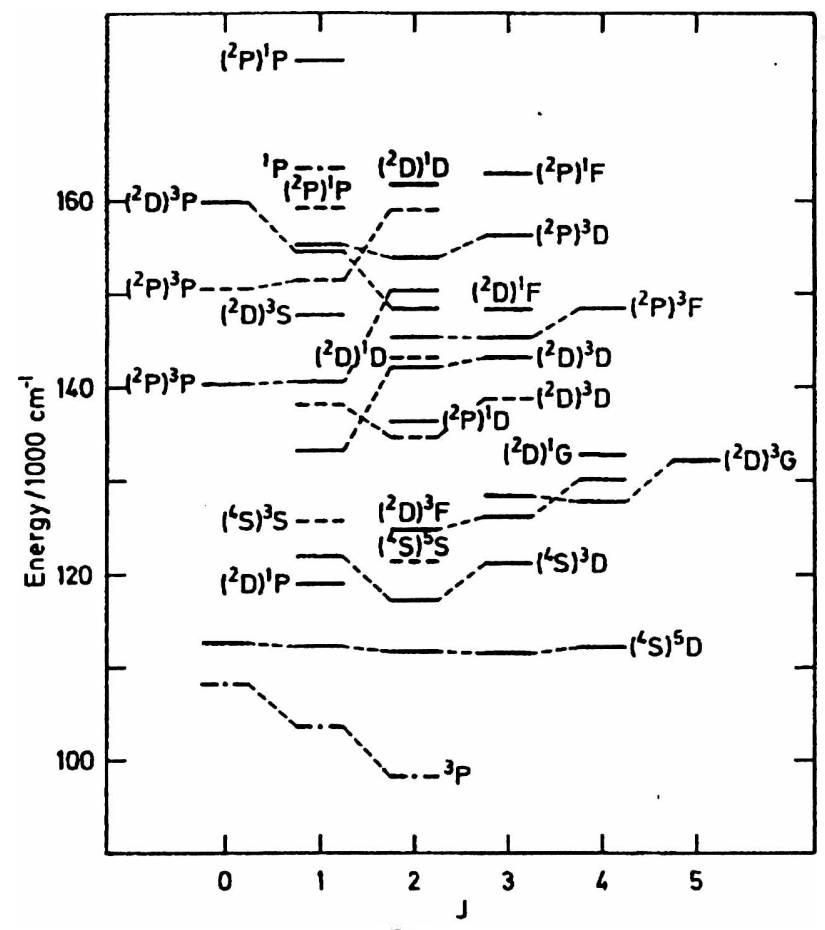

Fig. 3. Structure of the $5 s 5 p^{5}+5 s^{2} 5 p^{3}(5 d+6 s)$ configurations of Xe IIl. $5 p^{5}$ levels are indicated by broken lines with dots in the centre, $6 s$ levels by dashed and $S d$ by fully drawn lines. All levels are given in the $L S$ coupling scheme.

particular the $4 d \leftrightarrow 5 d$ interaction, in the theoretical predictions of the level structure.

In Xe III the deviation between the observed and the calculated positions of the $5 p^{3} 5 d^{3} S_{1}$ level is $700 \mathrm{~cm}^{-1}$, even when using fitted values of the energy parameters. When introducing the Rydbergseries configuration interaction the

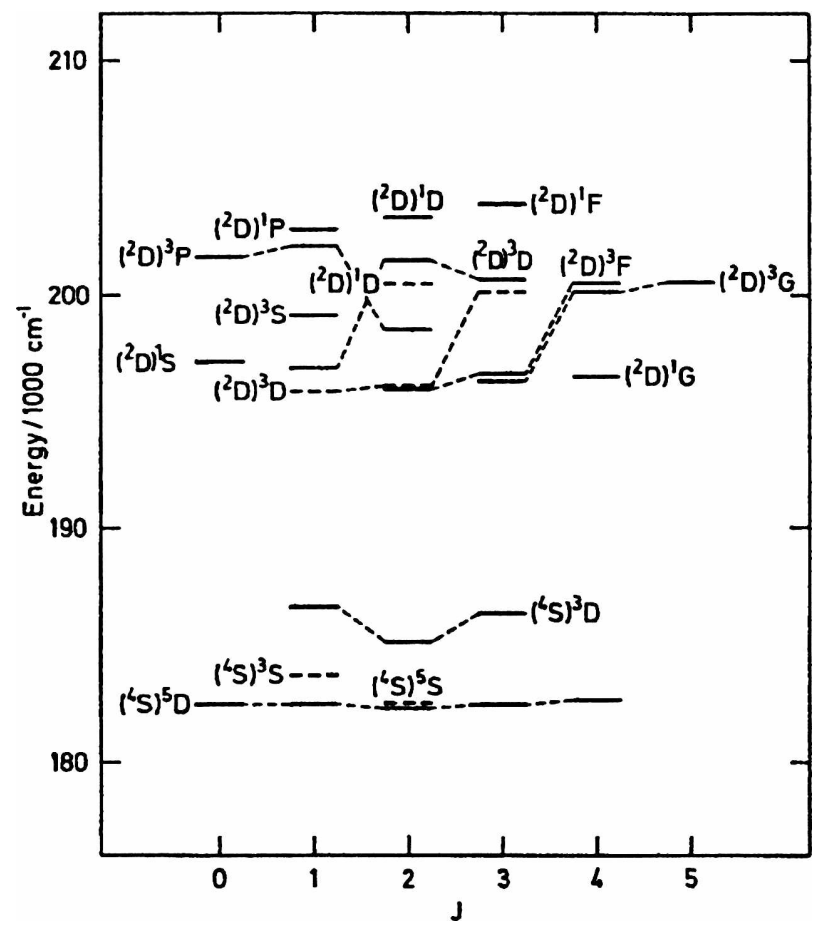

Fig. 4. Structure of the $5 s^{2} 5 p^{3}(6 d+7 s)$ configurations of Xe III. Only levels based on the ${ }^{4} S$ and ${ }^{2} D$ parent terms are experimentally established and indicated in the figure. $6 d$ levels are indicated by fully drawn lines and $7 s$ levels by dashed lines. All levels are given in the $L S$ coupling scheme. deviation decreases to $170 \mathrm{~cm}^{-1}$. At the same time the overall mean error of the fit decreases by approximately $20 \%$. The $S d \leftrightarrow 6 d R^{3} \mathrm{CI}$ integral could not be treated as an adjustable parameter at the same time as the $R^{\prime}$ and $R^{2} \mathrm{Cl}$ integrals. The $R^{3}$ integral was therefore optimized in a series of separate calculations and kept fixed in the final calculation.

In general there is good agreement between the $g$, factors determined in the least-square fit and those obtained experimentally by Humphreys et al. [3] (Table VI). We have no reasonable explanation for the small observed $g$, factors of the two $J=1$ levels at 133234 and $138145 \mathrm{~cm}^{-1}$.

\section{Discussion}

Recently, much effort has been devoted to the construction of VUV lasers. One recently observed [21] VUV laser transition is the transition at $1089 \AA$ in $\mathrm{Xe}^{2+}$ connecting the odd level at $119026 \mathrm{~cm}^{-1}$ above the ground state, and the even-parity $5 s^{0} 5 p^{6}{ }^{1} S_{0}$ state at $210857 \mathrm{~cm}^{-1}$. The lower state, previously designated as $5 s 5 p^{5}{ }^{1} P_{1}$, is considered to decay rapidly to the ground state while the upper state can be populated by Auger processes.

As already pointed out, there is considerable mixing between the $5 s 5 p^{5}$ and the $5 s^{2} 5 p^{3} 5 d$ states, and in the present analysis the lower level has been designiated $5 s^{2} 5 p^{3}\left({ }^{2} D\right) 5 d^{1} P_{1}$. The purity of the state is only $44 \%$ and the $5 s 5 p^{5}{ }^{1} P_{1}$ contribution is $28 \%$. The $5 s 5 p^{5}{ }^{1} P_{1}$ state is mixed into a number of different $5 d$ states and this opens many different decay modes for the upper $5 s^{0} 5 p^{6}{ }^{1} S_{0}$ state. This fact probably has to be taken into account when discussing the possible efficiency of the laser action of this particular transition.

The present analysis, in particular as regards the mixing between the $5 s 5 p^{5}$ and the $5 s^{2} 5 p^{3} 5 d$ states, also has consequences for the intrepretation of the Auger spectrum of xenon following the ionization of a $4 d$ electron, the $\mathrm{N}_{4.5} \mathrm{OO}$ spectrum (Fig. 5). The spectrum shown was recorded by Werme et al. [22], but has also been extensively studied by Southworth et al. [23], and Aksela et al. [24].

The spectrum consists of lines corresponding to the $\mathrm{Xe}^{2+}$ ion being left in different final states. There are two lines possible for each final state, corresponding to the fine structure of the initial hole in the $4 d$ shell. One group of strong lines corresponds to the ion being left in the $5 s^{2} 5 p^{4}$ configuration, another group to the $5 s 5 p^{5}$ final states and a third group corresponds to the ion being left with an empty $5 s$ shell, i.e., the configuration $5 s^{0} 5 p^{6}$. The additional strong lines are satellites and are mainly caused by final-state configuration interaction, i.e., in the terminology of the present study, by the mixing between the $5 s 5 p^{5}$ and the $5 s^{2} 5 p^{3} 5 d$ (and possibly 6s) states.

A detailed comparison between the Auger data and the present optical data is given in Table VIII. The energy of the $5 s^{2} 5 p^{4}{ }^{3} P_{2}$ ground level is set to zero. The agreement in relative energies is very good; the deviation never exceeding the estimated uncertainties in the Auger values $(\approx 0.05 \mathrm{eV})$. The largest discrepancy is found for the $\left({ }^{2} P\right) 6 s^{1} P_{1}$ level. However, the identification of this state in the Auger spectrum is tentative as it is based on a single line. Moreover, this line is doubly classified. It can also be seen that those $5 d$ levels which have a significant $5 s 5 p^{5}$ contribution to the eigenvector give rise to strong satellite lines in the Auger spectrum.

The new classifications for the $\mathrm{Xe}^{2+}$ laser lines are sum- 


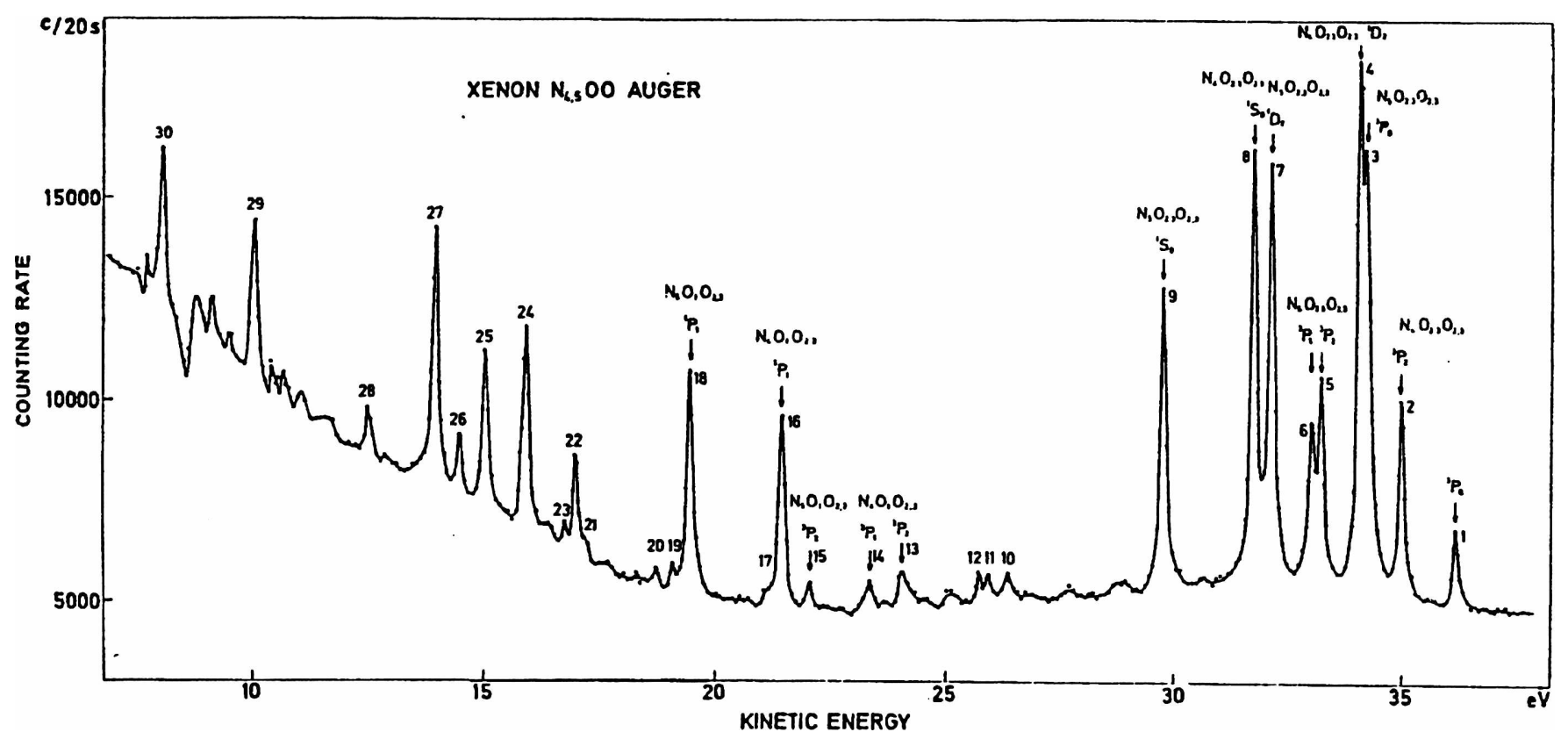

Fig. S. Xenon $\mathrm{N}_{4,} \mathrm{OO}$ Auger spectrum. from Ref. [22] (with permission from the authors).

marized in Table IX. The laser data are taken from the compilation by Beck et al. [9]. Table IX includes all laser lines ascribed with certainty or with some doubt to $\mathrm{Xe}^{2+}$. Only very few lines remain unclassified. We have also included a laser line at $3349 \AA$, which, with a question mark, has been ascribed to $\mathrm{Xe}^{3+}$, but in the present analysis has been classified as a $\mathrm{Xe}^{2+}$ line.

Based on a revised analysis and isoelectronic comparisons, Gallardo et al. [5] determined the value of $250400 \pm$ $300 \mathrm{~cm}^{-1}(31.05 \pm 0.04 \mathrm{eV})$ for the ionization energy of $\mathrm{Xe}^{2+}$. Their value, which was about $9000 \mathrm{~cm}^{-1}$ lower than the previously accepted value [25], is in fairly good agreement with the later value by Dutil and Marmet [26]. Using electronimpact ionization of xenon they arrived at the value of $31.24 \pm 0.10 \mathrm{eV}$. The present analysis does not indicate any need for revising the value of the ionization energy.

\section{Acknowledgements}

We are grateful for the financial support of the Consejo Nacional de Investigaciones Cientificas y Técnicas, Argentina and the Swedish Natural Science Research Council.

\section{References}

1. Boyce, J. C., Phys. Rev. 49, 730 (1936).

2. Humphreys, C. J., J. Res. Natl. Bur. Stand. 16, 639 (1936).

3. Humphreys, C. J., Meggers, W. F. and deBruin, T. L., J. Res. Natl. Bur. Stand. 23, 683 (1939).

4. Edlen, B., Phys. Rev. 65, 248 (1944).

5. Gallardo, M., Massone, C. A., Tagliaferri, A. A., Garavaglia, M. and Persson, W. Physica Scripta 19, 538 (1979).

6. Hansen, J. E. and Persson, W., Physica Scripta 25, 487 (1982).
7. Hertz, H., Z. Phys. A274, 289 (1975).

8. Hansen, J. E., Meijer, F. G., Outred, M., Persson, W. and Di Rocco, H. O., Physica Scripta 27, 254 (1983).

9. Beck, R., Englisch, W. and Gürs, K., Table of Laser Lines in Gases and Vapors, p. 7, Springer-Verlag Berlin, Heidelberg, New York (1978).

10. Persson, W. and Minnhagen, L., Ark. Fys. 37, 273 (1968)

11. Pettersson, S. -G., Physica Scripta 26, 296 (1982).

12. Minnhagen, L., Physica Scripta 11, 38 (1975).

13. Gallardo, M. and Reyna Almandos, J. G., Xenon Lines in the Range from $2000 \mathrm{~A}$ to $7000 \mathrm{~A}$. Serie "Monografias Cientificas" No. 1, Centro de Investigaciones Opticas, La Plata (1981).

14. Reyna Almandos, J. G., Gallardo, M. and Garavaglia, M., Opt. Pura Appl. 15, 1 (1982).

15. Humphreys, C. J., Unpublished.

16. Persson, W., Unpublished.

17. Persson, W. and Wahlstrōm, C. -G., Physica Scripta 31, 487 (1985)

18. Persson, W. and Wahlström, C. -G., Physica Scripta 30, 169 (1984).

19. Persson, W. and Reader, J., J. Opt. Soc. Am. B3, 959 (1986).

20. Persson, W. and Di Rocco, H. O.. Work in progress; Hansen. J. E., Judd, B. R., Lister, G. M. S. and Persson, W., J. Phys. B: At. Mol. Phys. 18, L275 (1985).

21. Kapteyn, H. C., Lee, R. W. and Falcone, R. W., Phys. Rev. Lett. 57, 2939 (1986).

22. Werme, L. O., Bergmark, T. and Siegbahn, K., Physica Scripta 6, 14! (1972).

23. Southworth, S., Becker, U., Truesdale, C. M., Kobrin, P. H., Lindle. D. W., Owaki, S. and Shiriey, D. A., Phys. Rev. A28, 261 (1983).

24. Aksela, H., Aksela, S. and Pulkkinen, H., Phys. Rev. A30, 865 (1984).

25. Moore, C. E., Jonization Potentials and lonization Limits Derived from the Analyses of Optical Spectra, Nat. Stand. Ref. Data Ser., Nat. Bur. Stand. NSRDS-NBS 34, Washington (1970).

26. Dutil, R. and Marmet, P., Int. J. Mass. Spectrom. \& Ion Phys. 35, 371 (I980).

27. Reyna Almandos, J. G., Bredice, F., Di Rocco, H. and Gallardo, M., Opt. Pura Appl. 18, 87 (1985). 
Table 1. Observed spectral lines of Xe III. Wavelengths in air Table I. Continued are given for lines with $\lambda>2000 A$. The intensity figures are visual estimates from the photographic density of the lines. An intensity figure in parenthesis indicales that the experimental data for the line stem from the unpublished wavelength list of Dr Humphreys. The calculated wavenumbers have been obtained from the level values given in Tables II and III by means of the Ritz combination principle. Only that part of the calculated wavenumber which differs from the observed is given. Level designations are given in LS notation

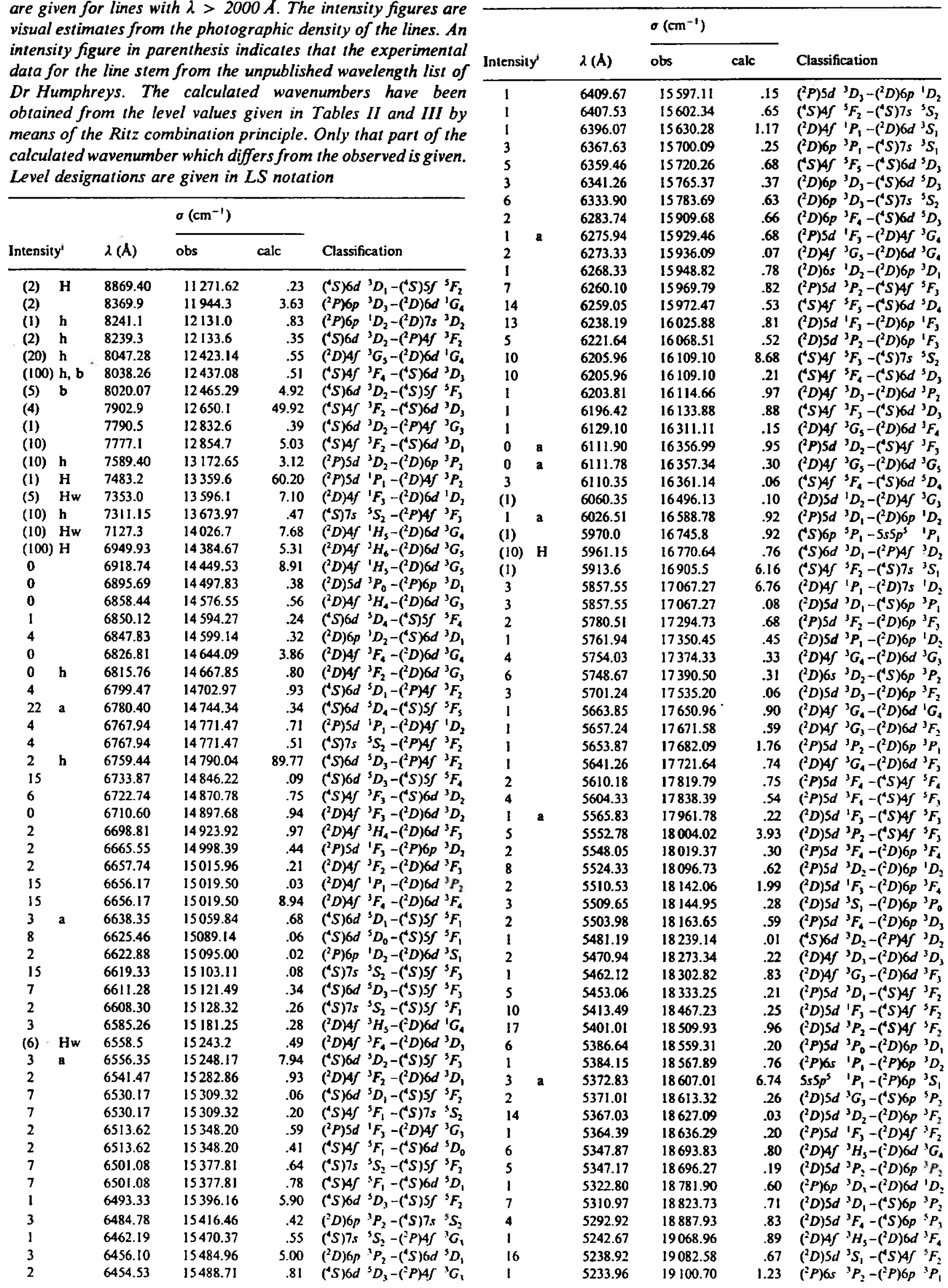




\begin{tabular}{|c|c|c|c|c|c|c|c|c|c|c|c|}
\hline \multirow{2}{*}{\multicolumn{2}{|c|}{ Intensily' }} & \multirow[b]{2}{*}{$\lambda(A)$} & \multicolumn{2}{|l|}{$\sigma\left(\mathrm{cm}^{-1}\right)$} & \multirow[b]{2}{*}{ Classification } & \multirow{2}{*}{\multicolumn{2}{|c|}{ Intensity' }} & \multirow[b]{2}{*}{$\lambda(\AA)$} & \multicolumn{2}{|l|}{$\sigma\left(\mathrm{cm}^{-1}\right)$} & \multirow[b]{2}{*}{ Classification } \\
\hline & & & obs & calc & & & & & obs & calc & \\
\hline 3 & & 5233.10 & 19103.81 & .73 & $\left({ }^{2} D\right) 5 d^{3} D_{3}-\left({ }^{2} D\right) 6 p{ }^{3} D_{2}$ & 4 & & 4387.47 & 22785.80 & .87 & $\left({ }^{2} P\right) 5 d^{3}$ \\
\hline 1 & & 5229.99 & 19115.17 & .03 & $\left({ }^{2} D\right) 4 f^{3} H_{5}-\left({ }^{2} D\right) 6 d^{3} G_{3}$ & 4 & & 4386.01 & 22793.35 & .48 & $5 s 5 p^{5} \quad 1$ \\
\hline II & & 5223.62 & 19138.51 & .51 & $\left({ }^{2} P\right) S d d^{3} F_{2}-\left({ }^{2} D\right) 6 p{ }^{1} F_{3}$ & 3 & & 4373.06 & 22860.88 & .93 & $\left.{ }^{2} D\right) 6 p{ }^{3} D_{2}-\left({ }^{4} S\right) 6 d{ }^{3} D_{2}$ \\
\hline 1 & & 5203.67 & 19211.88 & .77 & ${ }^{(2 D) 6 s}{ }^{1} D_{2}-\left({ }^{2} D\right) 6 p{ }^{3} D_{2}$ & 3 & & 4357.63 & 22941.83 & .80 & $\left({ }^{2} D\right) S d{ }^{3} F_{3}-\left({ }^{4} S\right) 6 p{ }^{3} P_{3}$ \\
\hline 1 & & 5148.03 & 19419.49 & .51 & $\left.{ }^{\left({ }^{2} P\right) S d}{ }^{\prime} F_{3}-{ }^{2} D\right) 4 f^{3} D_{3}$ & 2 & & 4319.85 & 23142.47 & .51 & $\left({ }^{4} S\right) 4 f{ }^{3} F_{2}-\left({ }^{2} D\right) 6 d{ }^{3} D_{1}$ \\
\hline 6 & & 5142.98 & 19438.59 & .57 & $\left({ }^{2} D\right) 5 d^{3} D_{3}-\left({ }^{2} D\right) 6 p^{3} F_{3}$ & 7 & & 4309.32 & 23199.03 & .03 & $\left({ }^{2} D\right) 5 d{ }^{3} D_{3}-\left({ }^{4} S\right) 4 f^{5} F_{4}$ \\
\hline 1 & & 5114.53 & 19546.72 & .61 & ( $\left.{ }^{2} D\right) 6 s s^{1} D_{2}-\left({ }^{2} D\right) 6 p{ }^{3} F_{3}$ & 6 & & 4308.01 & 23206.07 & .12 & $(2 D) 4 f^{3} G_{3}-\left({ }^{2} D\right) G d d^{3} D_{2}$ \\
\hline 14 & & 5107.33 & 19574.24 & .15 & ('D) $5 d^{3} D_{1}-\left({ }^{4} S\right) 6 p^{3} P_{0}$ & 6 & & 4308.01 & 23206.07 & .04 & $\left({ }^{2} P\right) 6 s{ }^{3} P_{2}-\left({ }^{2} P\right) 6 p{ }^{3} S_{1}$ \\
\hline 9 & & 5041.40 & 19830.25 & .25 & $\left.{ }^{2} P\right) S d{ }^{3} D_{1}-\left({ }^{2} P\right) 6 p{ }^{3} D_{1}$ & 1 & a & 4305.83 & 23217.82 & .82 & ( $D) S d d^{3} D_{3}-\left({ }^{4} S\right) 4 f{ }^{5} F_{3}$ \\
\hline 1 & b & 5040.06 & 19835.52 & 6.26 & $\left.{ }^{(} P\right) 6 p \quad D_{2}-\left({ }^{2} D\right) 6 d^{1} F_{3}$ & 1 & a & 4297.96 & 23260.34 & .82 & (2P) $6 p{ }^{3} D_{1}-\left({ }^{2} D\right) 6 d$ \\
\hline 2 & & 5038.62 & 19841.20 & 0.92 & $\left({ }^{2} P\right) S d{ }^{3} D_{2}-\left({ }^{4} S\right)^{4} f^{3} F_{2}$ & 4 & & 4289.33 & 23307.13 & .07 & ( $\left.\left.{ }^{D} D\right) 6 s^{\prime} D_{2}-{ }^{4} S\right) 4 f^{\prime}$ \\
\hline 3 & & 5023.01 & 19902.85 & 3.09 & $\left({ }^{2} D\right) 4 f^{\prime} P_{1}-\left({ }^{2} D\right) 6 d^{\prime} D_{2}$ & 5 & $\mathbf{a}$ & 4287.58 & 23316.65 & .56 & $\left({ }^{2} D\right) S d d^{3} P_{1}-\left({ }^{2} P\right) 6 p$ \\
\hline 23 & & 5008.53 & 19960.40 & .37 & $\left({ }^{2} P\right) S d{ }^{3} P_{1}-\left({ }^{2} D\right) 6 P{ }^{3} F_{2}$ & 20 & & 4285.89 & 23325.85 & .86 & $\left.{ }^{2} D\right) 6 s{ }^{1} D_{2}-\left({ }^{4} S\right) 4 f^{5} F_{3}$ \\
\hline 0 & & 4996.10 & 20010.02 & 9.98 & $\left({ }^{4} S\right) 4 f^{5} F_{3}-\left({ }^{4} S\right) 6 d{ }^{3} D_{3}$ & 4 & & 4278.91 & 23363.90 & .39 & $\left({ }^{2} P\right) 5 d{ }^{1} F_{3}-\left({ }^{2} P\right) 6 p{ }^{3}$ \\
\hline ] & & 4952.53 & 20186.06 & 5.89 & $\left({ }^{2} D\right) 4 f^{3} G_{3}-\left({ }^{2} D\right) 6 d{ }^{3} P_{2}$ & 4 & & 4274.14 & 23389.97 & .96 & $\left({ }^{2} D\right) 5 d d^{3} P_{1}-\left({ }^{2} P\right) 6 p{ }^{3}$ \\
\hline 5 & & 4927.51 & 20288.55 & .58 & $\left({ }^{2} D\right) 5 d{ }^{3} S_{1}-\left({ }^{2} D\right) 6 p{ }^{3} P_{1}$ & 17 & & 4272.58 & 23398.50 & .59 & $\left({ }^{2} D\right) 5 d{ }^{3} D_{3}-\left({ }^{2} D\right) 6 p$ \\
\hline 1 & a & 4926.72 & 20291.83 & .35 & $\left({ }^{2} D\right) 6 p{ }^{3} D_{2}-\left({ }^{4} S\right) 6 d{ }^{5} D_{1}$ & 12 & & 4263.40 & 23448.88 & .91 & $\left({ }^{2} P\right) 6 s{ }^{3} P_{2}-\left({ }^{2} D\right) 4 f$ \\
\hline 1 & & 4918.87 & 20324.22 & .16 & $\left.{ }^{2} D\right) 5 d d^{1} D_{2}-\left({ }^{2} P\right) 6 p{ }^{3} S_{1}$ & 2 & & 4246.38 & 23542.86 & .88 & $\left({ }^{2} D\right) 5 d^{3} D_{3}-\left({ }^{2} D\right) 6 p{ }^{3} D_{3}$ \\
\hline 2 & & 4881.05 & 20481.70 & .70 & $5 s s p^{s} \quad{ }^{1} P_{1}-\left({ }^{2} P\right) 6 p \quad{ }^{1} D_{2}$ & 16 & . & 4240.24 & 23576.96 & .99 & $\left.{ }^{2} D\right) 5 d^{1} F_{3}-\left({ }^{2} D\right) 6 p{ }^{1} D_{2}$ \\
\hline 21 & & 4869.40 & 20530.66 & .55 & (2D) $5 d{ }^{3} D_{2}-\left({ }^{2} D\right) 6 p{ }^{3} F_{3}$ & 2 & $\mathbf{a}$ & 4235.76 & 23601.90 & .77 & $\left({ }^{2} D\right) 6 s s^{3} D_{3}-\left({ }^{2} D\right) 6 p$ \\
\hline 8 & a & 4796.51 & 20842.69 & .65 & $\left({ }^{2} D\right) 5 d d^{3} F_{3}-\left({ }^{4} S\right) 6 p{ }^{5} P_{2}$ & 13 & & 4226.97 & 23650.97 & .91 & ( $\left.{ }^{2} D\right) 6 s{ }^{1} D_{2}-\left({ }^{2} D\right) 6 p{ }^{3}$ \\
\hline 25 & & 4794.49 & 20851.44 & .49 & $\left.{ }^{2} D\right) 6 s{ }^{3} D_{1}-\left({ }^{2} D\right) 6 p{ }^{3} D_{1}$ & 0 & & 4225.96 & 23656.63 & .76 & $\left({ }^{2} P\right) 5 d^{1} F_{3}-\left({ }^{2} D\right) 4 f^{3}$ \\
\hline 3 & h & 4781.08 & 20909.92 & .78 & $\left({ }^{2} P\right) 6 p^{3} D_{1}-\left({ }^{2} D\right) 7 s{ }^{3} D_{2}$ & 10 & & 4216.71 & 23708.53 & .57 & $\left({ }^{2} D\right) S d d^{3} G_{3}-\left({ }^{4} S\right) 6 p{ }^{3} P_{2}$ \\
\hline 10 & & 4757.32 & 21014.39 & .36 & $\left({ }^{2} P\right) S d{ }^{3} F_{3}-\left({ }^{4} S\right) 4 \int^{5} F_{4}$ & 24 & & 4213.99 & 23723.82 & .85 & $-\left({ }^{4} S\right) 4 f^{5} F_{2}$ \\
\hline 18 & & 4748.94 & 21051.47 & .60 & $\left({ }^{2} P\right) S d d^{1} F_{3}-\left({ }^{2} P\right) 6 p \quad{ }^{1} D_{2}$ & 14 & & 4209.58 & 23748.63 & .73 & $-\left({ }^{2} P\right) 6 p^{3}$ \\
\hline 10 & & 4743.90 & 21073.83 & .93 & $\left({ }^{2} P\right) S d{ }^{3} F_{2}-\left({ }^{4} S\right) 4 f^{5} F_{3}$ & 13 & & 4203.89 & 23780.83 & .72 & $\left({ }^{2} P\right) 5 d{ }^{3} P_{1}-\left({ }^{2} D\right) 6 p$ \\
\hline 16 & & 4723.60 & 21164.40 & .42 & $\left({ }^{4} S\right) 6 s^{3} S_{1}-\left({ }^{4} S\right) 6 p{ }^{5} P_{1}$ & 2 & a & 4202.39 & 23789.32 & .22 & $\left({ }^{2} D\right) 6 p{ }^{3} F_{3}-\left({ }^{4} S\right) 6 d^{3}$ \\
\hline 20 & b & 4712.58 & 21213.89 & .92 & $\left({ }^{2} P\right) S d d^{3} F_{3}-\left({ }^{2} D\right) 6 P^{3} F_{4}$ & 9 & & 4194.87 & 23831.96 & .89 & $\left.\left.{ }^{2} D\right) 6{ }^{1} D_{2}-{ }^{4} S\right) 4 f^{5} F_{2}$ \\
\hline 6 & & 4697.42 & 21282.35 & .41 & $\left.{ }^{2} D\right) 5 d^{3} D_{3}-\left({ }^{2} D\right) 6 p p^{1} F_{3}$ & 4 & & 4181.15 & 23910.15 & .08 & $\left({ }^{2} D\right) 5 d^{3} D_{3}-\left({ }^{2} D\right) 6 p^{3}$ \\
\hline 2 & $\mathbf{a}$ & 4685.19 & 21337.87 & .96 & $\left({ }^{2} P\right) 5 d{ }^{3} D_{2}-\left({ }^{2} P\right) 6 p{ }^{3} D_{1}$ & 16 & & 4176.53 & 23936.61 & .61 & $\left({ }^{2} D\right) 6 s{ }^{3} D_{3}-\left({ }^{2} D\right) 6 p{ }^{3}$ \\
\hline 20 & & 4683.55 & 21345.38 & .36 & $\left({ }^{4} S\right) 6 s{ }^{3} S_{1}-\left({ }^{4} S\right) 6 p p^{5} P_{2}$ & 2 & & 4167.60 & 23987.89 & .85 & (2D)6p $1 D_{2}-\left({ }^{2} D\right) 6 d^{3}$ \\
\hline 1 & $\mathbf{b}$ & 4680.73 & 21358.24 & .21 & $\left({ }^{2} P\right) S d{ }^{3} F_{3}-\left({ }^{2} D\right) 6 p{ }^{3} D_{3}$ & 2 & & 4162.37 & 24018.04 & .12 & $\left({ }^{2} D\right) 6 s{ }^{1} D_{2}-\left({ }^{2} D\right) 6 p^{3}$ \\
\hline 18 & a & 4673.67 & 21390.46 & .44 & $\left.{ }^{(} D\right) 6 s^{1} D_{2}-\left({ }^{2} D\right) 6 p D^{1} F_{3}$ & 5 & b & 4154.66 & 24062.61 & .73 & $\left.{ }^{(2} P\right) 5 d{ }^{3} L$ \\
\hline 3 & & 4671.81 & 21399.01 & 8.98 & ( $\left.{ }^{2} P\right) S d{ }^{3} F_{2}-\left({ }^{2} D\right) 6 p{ }^{3} D_{3}$ & 4 & b & 4152.74 & 24073.73 & .87 & $\left({ }^{2} P\right) 5 d{ }^{3} P_{0}-\left({ }^{2} D\right) 6 p$ \\
\hline 8 & & 4671.60 & 21399.98 & 400.37 & $\left({ }^{2} D\right) 5 d^{3} P_{0}-\left({ }^{2} P\right) 6 p p^{3} S_{1}$ & 8 & a & 4152.04 & 24077.78 & 8.05 & $\left({ }^{2} D\right) S d d^{\prime} D_{2}-\left({ }^{2} P\right) 6 p$ \\
\hline 17 & a & 4657.78 & 21463.47 & .45 & $\left({ }^{2} D\right) 6 s \quad{ }^{1} D_{2}-\left({ }^{2} D\right) 6 p{ }^{1} P_{1}$ & 3 & b & 4150.78 & 24085.10 & 4.77 & $\left({ }^{P} P\right)_{6 s} 1$ \\
\hline 1 & h & 4656.73 & 21 468.32 & .35 & $\left({ }^{2} D\right) 4 f^{3} D_{3}-\left({ }^{2} D\right) 6 d^{1} F_{3}$ & 22 & a & 4145.74 & 24114.33 & .48 & $-\left({ }^{2} D\right) 6 p^{3}$ \\
\hline 5 & & 4644.17 & 21526.33 & .27 & $\left({ }^{2} D\right) 6 p{ }^{3} D_{2}-\left({ }^{4} S\right) 7 s s^{3} S_{1}$ & 5. & & 4143.92 & 24124.92 & 5.34 & $\left({ }^{2} D\right) 6 s^{1}$ \\
\hline 1 & & 4643.60 & 21529.01 & .04 & $\left({ }^{2} P\right) 5 d{ }^{3} P_{1}-\left({ }^{2} D\right) 6 p{ }^{3} D_{2}$ & 13 & b & 4141.99 & 24136.16 & .13 & $\left({ }^{2} P\right) S d{ }^{3} D_{2}-\left({ }^{2} P\right) 6 p$ \\
\hline 15 & & 4641.40 & 21539.18 & .18 & $\left({ }^{2} P\right) S d{ }^{3} F_{3}-\left({ }^{4} S\right) 4 \int^{3} F_{2}$ & 12 & & 4132.40 & 24192.21 & .41 & $\left({ }^{2} D\right) 5 d d^{3} S_{1}-\left({ }^{2} D\right) 6 p$ \\
\hline 8 & & 4632.64 & 21579.95 & .96 & (2P) $5 d{ }^{3} F_{2}-\left({ }^{3} S\right) 4 f^{3} F_{2}$ & 12 & & 4110.05 & 24323.78 & .82 & ${ }_{2}-\left({ }^{2} D\right) 6 p^{3}$ \\
\hline 2 & a & 4631.65 & 21584.56 & .65 & $\left({ }^{2} D\right) 4 f^{3} G_{4}-\left({ }^{2} D\right) 6 d^{3} G_{3}$ & 20 & & 4109.08 & 24329.51 & .56 & $2-\left({ }^{2} D\right) 6 p^{3}$ \\
\hline 6 & & 4620.42 & 21636.98 & .95 & $\left({ }^{2} P\right) S d \quad F_{3}-\left({ }^{2} P\right) 6 p{ }^{3} D_{3}$ & 5 & b & 4095.03 & 24412.98 & .88 & ${ }_{2}-\left({ }^{2} D\right) 4 f^{3}$ \\
\hline 2 & & 4614.83 & 21663.19 & 2.97 & $\left({ }^{2} D\right) S d d^{1} D_{2}-\left({ }^{2} D\right) 4 f^{1} P_{1}$ & 21 & & 4078.70 & 24510.72 & .90 & $\left({ }^{2} D\right) 5 d^{\prime}$ \\
\hline 2 & & 4601.60 & 21725.47 & .41 & $\left({ }^{2} P\right) S d{ }^{3} F_{3}-\left({ }^{2} D\right) 6 p^{3} P_{2}$ & 7 & & 4072.97 & 24545.20 & 4.85 & $\left({ }^{2} P\right) 6 s^{3}$ \\
\hline 2 & $\mathbf{h}$ & 4592.98 & 21766.29 & .19 & $\left({ }^{2} P\right) S d{ }^{3} F_{2}-\left({ }^{2} D\right) 6 p{ }^{3} P_{2}$ & 25 & & 4060.45 & 24620.88 & .92 & $\left({ }^{2} P\right)_{6 s}$ \\
\hline 2 & & 4578.03 & 21837.33 & .32 & $\left({ }^{2} D\right) 5 d^{\prime} F_{3}-\left({ }^{4} S\right) 4 f^{3} F_{3}$ & 5 & & 4058.15 & 24634.84 & .85 & $\int_{2}-\left({ }^{2} D\right) 6 p^{3} D$ \\
\hline 7 & & 4569.12 & 21879.95 & 80.02 & $\left({ }^{2} D\right) 5 d{ }^{3} P_{2}-\left({ }^{4} S\right) 4 f^{3} F_{3}$ & 22 & & 4050.07 & 24683.98 & 4.04 & $-\left({ }^{4} s\right) 6 p$ \\
\hline 1 & $\mathbf{a}$ & 4555.51 & 21945.28 & .39 & ${ }^{(2 D)} 6 p p^{1} F_{3}-\left({ }^{4} S\right) 6 d^{3} D_{3}$ & 14 & & 4043.23 & 24725.74 & .85 & $\left.{ }^{2} P\right)_{6 s}{ }^{3}$ \\
\hline 9 & a & 4537.38 & 22032.99 & 3.10 & $\left({ }^{2} D\right) 6 s{ }^{3} D_{3}-\left({ }^{2} D\right) 6 p{ }^{3} F_{2}$ & 4 & a & 4032.91 & 24789.01 & .26 & $\left({ }^{2} D\right) 6 p^{3}$ \\
\hline 2 & h & 4528.24 & 22077.47 & .52 & $\left({ }^{2} P\right) 6 p{ }^{3} D_{2}-\left({ }^{2} D\right) 7 s^{3} D_{3}$ & 1 & & 4030.46 & 24804.08 & .28 & $\left.{ }^{2} D\right) 5 d{ }^{\prime} L$ \\
\hline 2 & b & 4528.24 & 22077.47 & .49 & $\left.{ }^{2} D\right) 6 p{ }^{3} P_{1}-\left({ }^{4} S\right) 6 d{ }^{3} D_{1}$ & 15 & & 4028.56 & 24815.78 & .82 & $\left(^{2} D\right) 5 d^{3} L$ \\
\hline 1 & & 4525.63 & 22090.20 & .16 & $\left({ }^{2} D\right) 5 d{ }^{3} F_{2}-\left({ }^{4} S\right) 6 p{ }^{5} P_{1}$ & 2 & & 4026.82 & 24826.50 & .92 & ( $\left.{ }^{2} P\right) 5 d{ }^{3} P_{2}-\left({ }^{2} P\right) 6 p{ }^{3} D_{1}$ \\
\hline 14 & $\mathbf{a}$ & 4503.41 & 22199.20 & .12 & $\left({ }^{2} D\right) 5 d^{1} D_{2}-\left({ }^{2} P\right) 6 p D^{\prime} D_{2}$ & 7 & & 4021.60 & 24858.72 & .73 & $\left({ }^{4} S\right) 5 d{ }^{3} D_{1}-\left({ }^{4} S\right) 6 p{ }^{5} P_{1}$ \\
\hline 5 & $\mathbf{a}$ & 4492.80 & 22251.62 & .69 & $\left({ }^{2} P\right) 6 S{ }^{3} P_{1}-\left({ }^{4} S\right) 4 f^{3} F_{2}$ & 4 & & 3998.55 & 25002.02 & .06 & $\left({ }^{2} D\right) 5 d{ }^{3} D_{2}-\left({ }^{2} D\right) 6 p{ }^{3} P_{2}$ \\
\hline 5 & a & $\mathbf{4 4 9 2 . 8 0}$ & 22251.62 & .66 & $\left({ }^{2} D\right) 4 f^{3} F_{2}-\left({ }^{2} D\right) 6 d d^{1} F_{3}$ & 21 & & 3992.85 & 25037.71 & .80 & $2-\left({ }^{2} D\right) 6 p^{3} P_{1}$ \\
\hline 4 & a & 4488.90 & 22270.95 & 1.09 & $\left({ }^{2} D\right) S d{ }^{3} F_{2}-\left({ }^{4} S\right) 6 p{ }^{5} P_{2}$ & 3 & & 3992.54 & 25039.65 & .66 & $-\left({ }^{4} s\right) 6 p^{3}$ \\
\hline 2 & & 4480.05 & 22314.95 & .98 & $\left({ }^{4} S\right)^{4} \int^{3} F_{4}-\left({ }^{2} D\right) 6 d{ }^{3} G_{3}$ & 12 & & 3985.96 & 25080.99 & 1.00 & $\left({ }^{2} P\right) 6 s s^{3} P_{2}-\left({ }^{2} P\right) 6 p$ \\
\hline 8 & b & 4470.90 & 22360.61 & .63 & ${ }_{s s s p^{5}} \quad{ }^{1} P_{1}-\left({ }^{2} P\right) 6 p \quad{ }^{2} P_{1}$ & 8 & & 3969.91 & 25182.39 & .45 & $\left.{ }^{2} D\right) 5 d d^{1} D_{2}-\left({ }^{2} D\right) 4 f^{3} D_{2}$ \\
\hline 5 & & 4468.16 & 22374.33 & .38 & $\left.{ }^{2} D\right) 5 d{ }^{3} D_{2}-\left({ }^{2} D\right) 6 p{ }^{1} F_{3}$ & 8 & & 3965.45 & 25210.71 & .76 & $\left({ }^{2} P\right) 5 d{ }^{3} P_{1}-\left({ }^{2} D\right) 6 p{ }^{3} P_{0}$ \\
\hline 7 & & 4453.60 & 22447.47 & .39 & 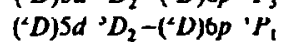 & 25 & & 3950.59 & 25305.54 & .54 & $\left({ }^{4} s\right) 6 s{ }^{5} S_{2}-\left({ }^{4} S\right) 6 p{ }^{5} P_{1}$ \\
\hline$i$ & & 4453.29 & 22449.03 & .24 & $\left({ }^{2} P\right) S d{ }^{1} F_{3}-\left({ }^{2} D\right) 4 f^{3} F_{4}$ & 3 & & 3950.12 & 25308.55 & .56 & $\left.{ }^{2} P\right) 6 p p^{3} D_{1}-\left({ }^{2} D\right) 7 s^{1} D_{2}$ \\
\hline is & & 4434.17 & 22545.83 & .81 & $\left({ }^{2} D\right) 6 s^{3} D_{1}-\left({ }^{2} D\right) 6 p{ }^{3} F_{2}$ & 3 & & 3941.50 & 25363.90 & .99 & $\left({ }^{2} D\right) 5 d{ }^{3} P_{2}-\left({ }^{4} S\right) 4 f^{3} F_{2}$ \\
\hline 3 & & 4417.97 & 22628.50 & .43 & $\left({ }^{2} P\right) 5 d{ }^{3} D_{1}-\left({ }^{2} P\right) 6 p{ }^{3} P_{1}$ & 2 & & 3932.81 & 25419.94 & 20.11 & $\left({ }^{2} P\right) 6 p^{3} D_{2}-\left({ }^{2} D\right) 6 d d^{1} D_{2}$ \\
\hline 2 & & 4417.78 & 22629.48 & .50 & $\left.{ }^{(2} P\right) S d{ }^{1} D_{2}-\left({ }^{2} D\right) 6 p{ }^{3} D_{1}$ & 25 & & 3922.55 & 25486.43 & .47 & $\left({ }^{4} S\right) 6 s{ }^{5} S_{2}-\left({ }^{4} S\right) 6 p{ }^{3} P_{2}$ \\
\hline 8 & & 4413.06 & 22653.67 & .63 & $\left({ }^{2} P\right) 5 d{ }^{3} D_{1}-\left({ }^{2} P\right) 6 p{ }^{3} P_{0}$ & 8 & & 3915.31 & 25533.55 & .69 & $\left({ }^{2} D\right) 5 d^{1} F_{3}-\left({ }^{4} S\right) 4 f^{3} F_{4}$ \\
\hline 13 & & 4395.17 & 22745.84 & .97 & $\left({ }^{2} P\right) 6 s \quad{ }^{1} P_{1}-\left({ }^{2} P\right) 6 p{ }^{3} S_{1}$ & 8 & & 3903.67 & 25609.69 & .77 & $\left({ }^{2} D\right) 5 d^{3} F_{2}-\left({ }^{4} S\right) 6 p{ }^{3} P_{1}$ \\
\hline 4 & & 4387.72 & 22784.50 & .46 & $\left.{ }^{2} D\right) 5 d d^{1} D_{2}-\left({ }^{2} P\right) 6 p{ }^{3} D_{3}$ & 19 & & 3895.05 & 25666.36 & .34 & $\left({ }^{2} P\right) 6 s \quad{ }^{3} P_{2}-\left({ }^{2} P\right) 6 p{ }^{3} D_{3}$ \\
\hline
\end{tabular}


Table I. Continued

\begin{tabular}{|c|c|c|c|c|c|}
\hline \multirow{2}{*}{\multicolumn{2}{|c|}{ Intensity }} & \multirow[b]{2}{*}{$\lambda(\AA)$} & \multicolumn{2}{|l|}{$\sigma\left(\mathrm{cm}^{-1}\right)$} & \multirow[b]{2}{*}{ Classification } \\
\hline & & & obs & calc & \\
\hline \multicolumn{2}{|l|}{2} & 3890.98 & 25693.21 & 2.74 & $\left({ }^{2} D\right) 6 p{ }^{3} F_{2}-\left({ }^{4} S\right) 6 d{ }^{3} D_{3}$ \\
\hline \multicolumn{2}{|l|}{13} & 3884.99 & 25732.82 & .84 & $\left({ }^{4} S\right) 5 d^{3} D_{3}-\left({ }^{4} S\right) 6 p{ }^{5} P_{2}$ \\
\hline \multicolumn{2}{|l|}{18} & 3880.46 & 25762.86 & .97 & $\left({ }^{2} D\right) 5 d{ }^{3} D_{1}-\left({ }^{2} D\right) 6 p{ }^{3} D_{1}$ \\
\hline \multicolumn{2}{|l|}{20} & 3877.82 & 25780.40 & .44 & $\left.{ }^{2} D\right) 6 s^{3} D_{3}-\left({ }^{2} D\right) 6 p{ }^{1} F_{3}$ \\
\hline \multicolumn{2}{|l|}{7} & 3861.51 & 25889.29 & .42 & $\left({ }^{2} P\right) 6 p{ }^{3} D_{2}-\left({ }^{2} D\right) 6 d^{1} F_{3}$ \\
\hline \multicolumn{2}{|l|}{19} & 3861.04 & 25892.44 & .49 & $\left({ }^{2} P\right) S d{ }^{1} D_{2}-\left({ }^{2} D\right) 6 p{ }^{3} D_{2}$ \\
\hline \multicolumn{2}{|l|}{2} & 3860.19 & 25898.14 & 7.85 & $\left({ }^{2} D\right) 6 p{ }^{3} F_{2}-\left({ }^{4} S\right) 6 d^{3} D_{1}$ \\
\hline 14 & & 3854.28 & 25937.85 & .95 & $\left({ }^{2} D\right) 5 d{ }^{3} F_{3}-\left({ }^{4} S\right) 6 p^{3} P_{2}$ \\
\hline 8 & & 3847.40 & 25984.23 & .33 & $\left({ }^{2} P\right) S d{ }^{3} D_{3}-\left({ }^{2} D\right) 4 f^{3} D_{3}$ \\
\hline 18 & & 3841.87 & 26021.63 & .73 & $\left({ }^{2} D\right) 5 d^{3} D_{2}-\left({ }^{2} D\right) 6 p{ }^{3} P_{1}$ \\
\hline 20 & & 3841.53 & 26023.94 & .88 & $\left({ }^{2} D\right) 6 s^{3} D_{2}-\left({ }^{2} D\right) 6 p{ }^{3} F_{2}$ \\
\hline 8 & & 3829.71 & 26104.26 & .07 & $\left({ }^{4} S\right) 4 f^{3} F_{4}-\left({ }^{2} D\right) 6 d{ }^{3} G_{4}$ \\
\hline 3 & & 3823.15 & 26149.05 & .15 & $\left({ }^{2} P\right) S d{ }^{3} P$ \\
\hline 2 & & 3819.49 & 26174.10 & .06 & $5 s 5 p^{3} \quad ' F$ \\
\hline 7 & & 3816.78 & 26192.69 & .79 & $\left({ }^{2} P\right) S d{ }^{3} D_{1}-\left({ }^{2} D\right) 4 f^{3} F_{2}$ \\
\hline 3 & & 3811.74 & 26227.32 & .34 & $\left({ }^{2} P\right) 5 d^{1} D_{2}-\left({ }^{2} D\right) 6 p{ }^{3} F_{3}$ \\
\hline 4 & $\mathbf{w}$ & 3802.98 & 26287.73 & .92 & $-\left({ }^{2} D\right) 6 d{ }^{\prime} G_{4}$ \\
\hline 8 & & 3801.71 & 26296.51 & .66 & $-\left({ }^{2} D\right) 4 f^{\prime} D_{2}$ \\
\hline 2 & & 3792.74 & 26358.70 & .76 & $\left({ }^{4} S\right) 4 f^{3} F_{3}-\left({ }^{2} D\right) 6 d^{3} F_{3}$ \\
\hline 15 & & 3791.67 & 26366.14 & .16 & $\left({ }^{2} D\right) 6 s^{3} D_{1}-\left({ }^{2} D\right) 6 p{ }^{2} P_{1}$ \\
\hline 28 & & 3781.00 & 26440.54 & .66 & $\left.{ }^{4} S\right) 6 p{ }^{3} P_{2}$ \\
\hline 24 & & 3776.32 & 26473.31 & .50 & $\left({ }^{2} P\right) 6 s^{3}$ \\
\hline 2 & $\mathbf{b}$ & 3775.49 & 26479.13 & .16 & $-\left({ }^{2} D\right) 6 d{ }^{3} F_{4}$ \\
\hline 15 & & 3772.53 & 26499.91 & .86 & $-\left({ }^{2} P\right) 6 p{ }^{1} P_{1}$ \\
\hline 1 & & 3772.25 & 26501.87 & 2.15 & ${ }_{2}-\left({ }^{2} D\right) 6 d{ }^{3} P_{2}$ \\
\hline 12 & & 3768.93 & 26525.22 & .30 & $\left({ }^{2} D\right) 6 d{ }^{3} G_{3}$ \\
\hline 14 & & 3765.85 & 26546.91 & .90 & $\left({ }^{2} P\right) 6 p^{3} P_{1}$ \\
\hline 19 & & 3762.28 & 26572.10 & .10 & $\left({ }^{2} P\right) 6 s{ }^{3} F$ \\
\hline 5 & & 3757.98 & 26602.51 & .58 & $\left({ }^{2} D\right) 5 d{ }^{1} D_{2}-\left({ }^{2} D\right) 4 f^{3} P_{2}$ \\
\hline 3 & & 3751.44 & 26648.88 & .91 & $\left({ }^{2} P\right) 5 d^{3} F_{3}-\left({ }^{2} D\right) 6 p{ }^{1} D_{2}$ \\
\hline 18 & & 3745.71 & 26689.65 & .69 & $\left.{ }^{2} D\right) 6 p{ }^{1} D_{2}$ \\
\hline 2 & & 3743.74 & 26703.69 & .70 & $\left.{ }^{2} D\right) 6 d{ }^{3} D_{3}$ \\
\hline 2 & & 3739.60 & 26733.25 & .24 & $\left({ }^{2} P\right) 5 d{ }^{3} D_{1}-\left({ }^{2} P\right) 6 p{ }^{3} S_{1}$ \\
\hline 5 & $\mathbf{b}$ & 3728.91 & 26809.89 & 10.19 & $\left.{ }^{2} P\right) 6 p{ }^{1} D_{2}-\left({ }^{2} P\right) 6 d{ }^{3} D_{1}$ \\
\hline 1 & & 3721.82 & 26860.96 & 1.03 & $\left.{ }^{2} P\right) 6 p{ }^{3} D_{1}$ \\
\hline 1 & & 3721.03 & 26866.66 & .57 & (2P)Sd 1 \\
\hline 10 & & 3711.91 & 26932.67 & .71 & $\left.{ }^{2} P\right) 6 p{ }^{3} P_{2}$ \\
\hline 8 & & 3708.94 & 26954.24 & .33 & $\left.{ }^{2} D\right) 4 f^{3} F_{2}$ \\
\hline 10 & & 3708.15 & 26959.98 & .93 & $\left.{ }^{2} P\right) 6 p{ }^{1} P_{1}$ \\
\hline 1 & & 3707.63 & 26963.76 & .76 & $\left.{ }^{2} P\right) 6 p{ }^{1} S_{0}$ \\
\hline 6 & & 3689.83 & 27093.83 & .91 & $\left({ }^{2} D\right) S d{ }^{3} D_{3}-\left({ }^{4} S\right) 4{ }^{3} F_{3}$ \\
\hline 3 & & 3687.04 & 27114.33 & .30 & $\left.{ }^{2} D\right) 6 p{ }^{1} D_{2}-\left({ }^{2} D\right) 6 d{ }^{3} S_{1}$ \\
\hline 23 & & 3676.63 & 27191.10 & .11 & $\left({ }^{4} S\right) 6 s{ }^{3} S_{1}-\left({ }^{4} S\right) 6 p{ }^{3} P_{0}$ \\
\hline 2 & & 3675.17 & 27201.90 & .95 & $\left.{ }^{2} D\right) 6 s^{1} D_{2}-\left({ }^{4} S\right) 4 f^{3} F_{3}$ \\
\hline 16 & & 3654.61 & 27354.93 & 5.06 & $\left({ }^{2} D\right) 6 p{ }^{3} P_{1}$ \\
\hline 8 & & 3653.09 & 27366.31 & .40 & $-\left({ }^{4} S\right) 6 p{ }^{3} P_{2}$ \\
\hline 13 & & 3649.57 & 27392.71 & .78 & $\left.{ }^{2} P\right) 6{ }^{3} P_{2}$ \\
\hline 10 & & 3644.14 & 27433.52 & .74 & $\left({ }^{2} D\right) 5 d{ }^{3} S_{1}-\left({ }^{2} P\right) 6 p{ }^{3} D_{1}$ \\
\hline 15 & & 3640.99 & 27457.26 & .29 & $\left({ }^{2} D\right) 5 d{ }^{3} D_{1}-\left({ }^{2} D\right) 6 p{ }^{3} F_{2}$ \\
\hline 11 & & 3636.02 & 27494.79 & .78 & $\left({ }^{2} D\right) S d{ }^{3} P$ \\
\hline 15 & & 3632.14 & 27524.16 & .02 & $\left({ }^{2} P\right) 6 s{ }^{3} P$ \\
\hline 8 & & 3628.52 & 27551.62 & .69 & $\left.{ }^{2} P\right) 5 d{ }^{3} P_{2}$ \\
\hline 25 & & 3624.06 & 27585.52 & .62 & $\left({ }^{4} s\right) 6 s{ }^{3} s_{2}$ \\
\hline 21 & $\mathbf{a}$ & 3623.13 & 27592.60 & .55 & $\left({ }^{2} D\right) 6 s{ }^{3} D_{2}$ \\
\hline 4 & & 3621.59 & 27604.34 & .25 & (2P)6s ${ }^{2} P_{1}$ \\
\hline 11 & & 3620.00 & 27616.46 & .42 & $(P) S d{ }^{3} D$ \\
\hline 8 & & 3618.86 & 27625.16 & .09 & (2P)Sd ${ }^{3} P_{2}$ \\
\hline 10 & & $36 ! 5.86$ & 27648.08 & .21 & $\left({ }^{2} P\right) S d{ }^{3} P_{0}-\left({ }^{2} D\right) 6 p{ }^{3} P_{1}$ \\
\hline $\mathbf{0}$ & & 3610.97 & 27685.52 & 6.16 & $\left({ }^{2} P\right) 6 s{ }^{3} P_{2}-\left({ }^{2} D\right) 4 f^{3} F_{3}$ \\
\hline 22 & $\mathbf{a}$ & 3609.46 & 27697.10 & .07 & $\left({ }^{2} D\right) 6 s^{3} D_{3}-\left({ }^{4} S\right) 4 f^{5} F_{4}$ \\
\hline 8 & $\mathbf{h}$ & 3609.07 & 27700.09 & .50 & $\left({ }^{2} P\right) S d{ }^{3} D_{2}-\left({ }^{2} D\right) 4 f^{3} F_{2}$ \\
\hline 22 & $\mathbf{a}$ & 3607.02 & 27715.84 & .86 & $\left({ }^{2} D\right) 6 s{ }^{3} D_{3}-\left({ }^{4} S\right) 4 f^{5} F_{3}$ \\
\hline 12 & & 3601.87 & 27755.46 & .46 & $\left({ }^{2} D\right) 5 d{ }^{1} P_{1}-(S) 6 p{ }^{5} P_{1}$ \\
\hline 17 & & 3596.59 & 27796.21 & .20 & $\left({ }^{2} D\right) 6 s^{3} D_{1}-\left({ }^{2} D\right) 6 p^{3} P_{0}$ \\
\hline 5 & & 3593.38 & 27821.04 & .05 & $\left({ }^{2} D\right) 6 p{ }^{3} P_{1}-\left({ }^{2} D\right) 7 s^{3} D_{1}$ \\
\hline 8 & & 3591.98 & 27831.88 & .99 & $\left({ }^{4} S\right) S d{ }^{3} D_{3}-\left({ }^{4} S\right) 6 p{ }^{5} P_{3}$ \\
\hline 5 & & 3584.27 & 27891.75 & .48 & $\left({ }^{2} D\right) S d \quad D^{1} D_{2}-\left({ }^{2} D\right) 4 \int^{3} P_{1}$ \\
\hline 5 & & 3584.27 & 27891.75 & .67 & $\left({ }^{2} D\right) 6 p{ }^{3} P_{1}-\left({ }^{2} D\right) 6 d{ }^{3} F_{2}$ \\
\hline
\end{tabular}

Table I. Continued

\begin{tabular}{|c|c|c|c|c|c|}
\hline \multirow{2}{*}{\multicolumn{2}{|c|}{ Intensity' }} & \multirow[b]{2}{*}{$\lambda(A)$} & \multicolumn{2}{|l|}{$\sigma\left(\mathrm{cm}^{-1}\right)$} & \multirow[b]{2}{*}{ Classification } \\
\hline & & & obs & calc & \\
\hline 25 & & 3583.65 & 27896.57 & .63 & $\left({ }^{2} D\right) 6 s s^{3} D_{3}-\left({ }^{2} D\right) 6 p{ }^{3} F_{4}$ \\
\hline 25 & & 3579.70 & 27927.36 & .40 & $\left.{ }^{(} D\right) 6 s^{3} D_{2}-\left({ }^{2} D\right) 6 p{ }^{3} F_{3}$ \\
\hline 6 & b & 3578.54 & 27936.41 & .39 & $\left.{ }^{2} D\right) 5 d d^{1} P_{1}-\left({ }^{4} S\right) 6 p{ }^{5} P_{2}$ \\
\hline 5 & & 3574.40 & 27968.76 & .96 & $\left.\left.{ }^{2} D\right) 5 d^{\prime} D_{2}-{ }^{2} D\right) 4 f^{\prime} F_{3}$ \\
\hline 2 & & 3568.63 & 28013.98 & 4.08 & $\left({ }^{2} D\right) 5 d^{\prime} D_{2}-\left(^{2} D\right) 4 f^{\prime} D$ \\
\hline 17 & & 3565.19 & 28041.01 & 0.91 & $\left({ }^{2} D\right) 6{ }^{3} D_{3}-\left({ }^{2} D\right) 6 p^{3} D_{3}$ \\
\hline 4 & & 3564.86 & 28043.61 & .63 & $\left({ }^{2} D\right) 6 p p^{1} D_{2}-\left({ }^{2} D\right) 7 s^{3} D_{3}$ \\
\hline 10 & & 3562.99 & 28058.33 & .74 & $\left({ }^{2} D\right) S d d^{3} P_{0}-\left({ }^{2} D\right) 4 f^{3} D_{1}$ \\
\hline 14 & & 3562.22 & 28064.39 & .33 & $\left.\left({ }^{2} P\right) 65{ }^{3} P_{2}-\left({ }^{2} D\right) 4\right)^{3} L$ \\
\hline 14 & & 3561.37 & 28071.09 & .17 & $\left({ }^{2} P\right) S d$ ' $D_{2}-\left({ }^{2} D\right) 6 p p^{\prime}$ \\
\hline 10 & & 3561.23 & 28072.19 & .04 & $\left({ }^{2} P\right) S d d^{3} D_{1}-\left({ }^{2} D\right) 4 f^{1}$ \\
\hline 18 & & 3552.12 & 28144.19 & .18 & $\left({ }^{(} P\right) 5 d \quad D_{2}-\left({ }^{2} D\right) 6 p{ }^{\prime} P$ \\
\hline 5 & & 3546.88 & 28185.77 & .89 & $\left({ }^{2} D\right) S d d^{3} D_{2}-\left({ }^{\prime} S\right) 4 f$ \\
\hline 10 & & 3544.86 & 28201.83 & .77 & (2P) $5 d d^{3} D_{3}-\left({ }^{2} P\right) 6 p^{3} L$ \\
\hline 20 & & 3542.35 & 28221.81 & .89 & $\left({ }^{2} D\right) 6 s^{3} D_{3}-\left({ }^{4} S\right) 4 f^{s}$ \\
\hline 12 & & 3539.94 & 28241.02 & 0.94 & $\left({ }^{2} P\right) 5 d d^{3} D_{2}-\left({ }^{2} P\right) 6 p{ }^{3}$ \\
\hline 12 & & 3539.94 & 28241.02 & .82 & $-\left({ }^{2} D\right) 6 d{ }^{3} P_{2}$ \\
\hline 7 & & 3531.82 & 28305.95 & 6.17 & $-5 s^{0} 5 p^{6}$ \\
\hline 3 & & 3531.62 & 28307.55 & .55 & $\left({ }^{2} P\right) 5 d \quad P_{1}-\left({ }^{2} P\right) 4 f^{3}$ \\
\hline 16 & & 3522.80 & 28378.42 & .34 & $(S) S d d^{3} D_{1}-\left({ }^{4} S\right) 6 p^{3}$ \\
\hline 11 & & 3519.11 & 28408.18 & .12 & $\left({ }^{2} D\right) 6 s s^{3} D_{3}-\left({ }^{2} D\right) 6 p^{3}$ \\
\hline 13 & b & 3509.77 & 28483.78 & .81 & $\left({ }^{2} P\right) 5 d d^{3} D_{2}-\left({ }^{2} D\right) 4 f^{3}$ \\
\hline 11 & & 3501.65 & 28549.82 & .89 & $\left.{ }^{(} D\right) 6 p{ }^{1} D_{2}-\left({ }^{2} D\right) 7 s$ \\
\hline 4 & & 3494.82 & 28605.62 & .62 & $\left({ }^{2} P\right) 5 d d^{3} F_{3}-(S) 4 f^{3}$ \\
\hline 8 & & 3494.51 & 28608.16 & .20 & $(P P) S d{ }^{3} D_{1}-\left({ }^{2} P\right) 6 p$ \\
\hline 6 & & 3488.13 & 28660.48 & .40 & $2-\left({ }^{2} D\right) 6 d^{3}$ \\
\hline 4 & & 3479.14 & 28734.54 & .59 & $\left.{ }^{(} D\right) 6 s^{3}$ \\
\hline 10 & & 3472.35 & 28790.72 & .63 & $-\left({ }^{2} D\right) 6 d^{3} D_{1}$ \\
\hline 14 & & 3468.22 & 28825.01 & .15 & $(' s) 6 s$ \\
\hline 15 & & 3467.22 & 28833.32 & .58 & $\left({ }^{2} D\right) 5 d^{3}$ \\
\hline 15 & & 3467.22 & 28833.32 & .58 & (2D)Sd ${ }^{3} F$ \\
\hline 16 & & 3454.27 & 28941.41 & .62 & $-\left({ }^{2} D\right) 6 p$ \\
\hline 11 & & 3451.23 & 28966.90 & 7.69 & $\left({ }^{2} D\right) 5 d^{3}$ \\
\hline 15 & $\mathbf{a}$ & 3444.39 & 29024.43 & .38 & $\left({ }^{2} P\right) 6 s$ \\
\hline 20 & $\mathbf{a}$ & 3444.24 & 29025.69 & .95 & $\left({ }^{2} D\right) 5 d^{3}$ \\
\hline 8 & & 3435.74 & 29097.50 & .58 & $\left(S^{2}\right) 4 f^{3}$ \\
\hline 2 & & 3414.54 & 29278.15 & .56 & $-\left({ }^{2} D\right) 6 d^{3}$ \\
\hline 8 & & 3403.91 & 29369.58 & .73 & $\left.{ }^{2} D\right) 5 d^{3}$ \\
\hline 7 & & 3399.87 & 29404.48 & .34 & $\left({ }^{2} P\right) 6 s$ \\
\hline 2 & b & 3395.53 & 29442.06 & 1.82 & $\left({ }^{2} D\right) 6 p{ }^{3} L$ \\
\hline 3 & & 3392.58 & 29467.66 & .71 & ( $\left.{ }^{2} P\right)^{5} d^{\prime} / F$ \\
\hline 10 & & 3390.64 & 29484.52 & .46 & $(P P) 6 s$ \\
\hline 4 & & 3386.23 & 29522.92 & 38 & $2-\left({ }^{2} D\right) 6 d^{3}$ \\
\hline 12 & b & 3384.10 & 29541.50 & .40 & $\left({ }^{C} S\right) S d d^{3} D_{2}-\left({ }^{\prime} S\right) 6 p p^{3}$ \\
\hline 10 & & 3383.92 & 29543.07 & 2.59 & $\left({ }^{2} D\right) 6 p^{3 / J}$ \\
\hline 10 & & 3383.92 & 29543.07 & .10 & $\left({ }^{2} D\right) 5 d^{\prime} /$ \\
\hline 4 & & 3381.64 & 29562.99 & .39 & $\left.{ }^{(} D\right) 6 p^{3}$ \\
\hline 9 & & 3379.03 & 29585.82 & .80 & $\left.{ }^{2} D\right) 5 d{ }^{3} P_{2}-\left({ }^{2} P\right) 6 p{ }^{3} D_{2}$ \\
\hline 3 & & 3376.98 & 29603.78 & .61 & $\left.\left({ }^{4} S\right) 4\right)^{3} F$ \\
\hline 9 & & 3370.65 & 29659.38 & .20 & $\left({ }^{2} D\right) 5 d^{3}$ \\
\hline 3 & & 3365.70 & 29703.00 & .09 & $(s) 4 f$ \\
\hline 3 & & 3365.32 & 29706. & .68 & $\left.{ }^{2} D\right) 6 p^{3}$ \\
\hline 2 & & 3363.50 & 29722.42 & .33 & $-(4 S) 6 p^{3} P_{2}$ \\
\hline 8 & & 3362.77 & 29728.87 & .83 & $(s) 4 f$ \\
\hline 3 & & 3358.50 & 29766.67 & .87 & $\left({ }^{4} s\right) 4 f$ \\
\hline 15 & & 3357.99 & 29771.19 & .23 & $-\left({ }^{2} D\right) 6 p p^{\prime} F_{3}$ \\
\hline 4 & & 3353.54 & 29810.70 & .30 & $\left.{ }^{2} D\right) 6 d d^{3} D_{1}$ \\
\hline 6 & & 3350.35 & 29839.08 & 8.96 & $\left.{ }^{2} D\right) 6 p^{3}$ \\
\hline 12 & & 3349.76 & 29844.33 & .24 & ${ }_{2}-\left({ }^{2} D\right) 6 p P^{1} P_{1}$ \\
\hline 2 & & 3345.49 & 29882.42 & 3.03 & $\left({ }^{2} D\right) S d d^{\prime} G$ \\
\hline 7 & & 3344.93 & 29887.43 & .44 & $(S) 4 f^{5} F$ \\
\hline 4 & & 3344.27 & 29893.32 & .25 & $\left({ }^{2} D\right) 5 d$ \\
\hline 16 & a & 3340.67 & 29925.54 & .56 & $-\left({ }^{2} D\right) 6 p$ \\
\hline 14 & a & 3340.37 & 29928.23 & .21 & $\left({ }^{2} P\right) 6 p{ }^{3} P_{2}$ \\
\hline 13 & & 3340.06 & 29931.00 & .03 & $-\left({ }^{2} P\right) 6 p{ }^{3} D_{1}$ \\
\hline 12 & b & 3339.50 & 29936.02 & 5.95 & $\left({ }^{2} D\right) 5 d^{3}$ \\
\hline 15 & a & 3338.99 & 29940.59 & .50 & $\left({ }^{2} D\right) 6{ }^{3} D$ \\
\hline 7 & & 3336.24 & 29965.27 & .35 & $\left.{ }^{2} D\right) 6 p{ }^{3} P_{0}$ \\
\hline
\end{tabular}


Table I. Continued

\begin{tabular}{|c|c|c|c|c|c|}
\hline \multirow{2}{*}{\multicolumn{2}{|c|}{ Intensity }} & \multirow[b]{2}{*}{$\lambda(\mathrm{A})$} & \multicolumn{2}{|l|}{$\sigma\left(\mathrm{cm}^{-1}\right)$} & \multirow[b]{2}{*}{ Classification } \\
\hline & & & obs & calc & \\
\hline 8 & & 3334.23 & 29983.34 & .25 & $\left.{ }^{2} D\right) 6 p^{3} F_{4}-\left({ }^{2} D\right) 6 d^{\prime} G_{4}$ \\
\hline 15 & $\mathbf{a}$ & 3331.65 & 30006.56 & .58 & $\left({ }^{2} P\right) S d{ }^{1} D_{2}-\left({ }^{4} S\right) 4 f^{5} F_{3}$ \\
\hline 5 & & 3326.39 & 30054.00 & .09 & $\left({ }^{2} D\right) 6 p{ }^{3} F_{4}-\left({ }^{2} D\right) 6 d{ }^{3} F_{3}$ \\
\hline 8 & & 3320.07 & 30111.21 & .24 & $(S) 4 \int^{3} F_{2}-\left({ }^{2} D\right) 6 d{ }^{1} F_{3}$ \\
\hline 8 & & 3320.07 & $30 ! 11.21$ & .27 & $-\left({ }^{2} D\right) 4{ }^{3} F_{2}$ \\
\hline 7 & & 3319.55 & 30115.93 & .90 & $\left.{ }^{2} P\right) S d{ }^{3} D_{2}-\left({ }^{2} P\right) 6 p$ ' $D_{2}$ \\
\hline 6 & & 3317.45 & 30134.99 & .97 & $\left({ }^{4} S\right) S d{ }^{3} D_{1}-\left({ }^{4} S\right) 6 p^{3} P_{2}$ \\
\hline 12 & & 3314.87 & 30158.45 & .52 & $\left({ }^{2} D\right) 5 d d^{3} S_{1}-\left({ }^{2} P\right) 6 p{ }^{3} D_{2}$ \\
\hline 6 & & 3314.26 & 30163.99 & 4.01 & $-\left({ }^{2} D\right) 6 d^{\prime} G_{4}$ \\
\hline 11 & $\mathbf{a}$ & 3306.80 & 30232.04 & 1.92 & $\left({ }^{2} D\right) 5 d{ }^{3} S_{1}-\left({ }^{2} P\right) 6 p{ }^{3} P_{1}$ \\
\hline 11 & $\mathbf{a}$ & 3306.46 & 30235.15 & 4.85 & $(S) 4 \int^{5} F_{3}-\left({ }^{2} D\right) 6 d{ }^{3} F_{3}$ \\
\hline 8 & & 3304.05 & 30257.20 & .12 & ( $D) S d{ }^{3} S_{1}-\left({ }^{2} P\right) 6 p{ }^{3} P_{0}$ \\
\hline 14 & & 3301.54 & 30280.20 & .16 & $(S) 6 p{ }^{3} P_{2}-\left({ }^{4} S\right) 6 d{ }^{3} D_{2}$ \\
\hline 14 & & 3295.94 & 30331.65 & .64 & $\left({ }^{2} P\right) 5 d{ }^{1} D_{2}-\left({ }^{2} D\right) 6 p{ }^{3} D_{3}$ \\
\hline 5 & & 3288.54 & 30399.90 & 400.07 & $\left({ }^{4} S\right) 4 f^{3} F_{3}-\left({ }^{2} D\right) 6 d{ }^{3} D_{3}$ \\
\hline 16 & - & 3287.91 & 30405.73 & .98 & $-\left({ }^{2} D\right) 6 d{ }^{3} P_{2}$ \\
\hline 13 & & 3287.80 & 30406.75 & .76 & $D_{2}-\left({ }^{4} S\right) 6 d{ }^{5} D_{3}$ \\
\hline 14 & & 3285.82 & 30425.07 & .02 & $-\left({ }^{4} s\right) 7 s^{5} S_{2}$ \\
\hline 8 & & 3284.64 & 30436.00 & 5.89 & $-\left({ }^{2} D\right) 4 f^{\prime} D_{2}$ \\
\hline 14 & & 3280.50 & 30474.41 & .34 & $\left({ }^{2} D\right) S d^{1} F_{3}-\left({ }^{2} D\right) 4 f^{3} G_{4}$ \\
\hline 3 & & 3279.13 & 30487.14 & .13 & $\left({ }^{2} P\right) S d{ }^{3} D_{1}-\left({ }^{2} P\right) 6 p{ }^{1} P_{1}$ \\
\hline 12 & & 3278.44 & 30493.55 & .60 & $\left({ }^{4} S\right) 6 p{ }^{3} P_{2}-\left({ }^{4} S\right) 6 d{ }^{5} D_{1}$ \\
\hline 13 & & 3276.39 & 30512.63 & .61 & $\left({ }^{2} P\right) S d{ }^{1} D_{2}-\left({ }^{4} S\right) d f^{3} F_{2}$ \\
\hline 3 & & 3269.40 & 30577.87 & .88 & $\left.{ }^{2} D\right) S d{ }^{3} D_{3}-\left({ }^{4} S\right) 4 f^{3} F_{2}$ \\
\hline 22 & & 3268.98 & 30581.79 & .78 & $\left.{ }^{4} S\right) 6 s{ }^{5} S_{2}-\left({ }^{4} S\right) 6 p{ }^{3} P_{2}$ \\
\hline 20 & & 3267.05 & 30599.86 & .75 & $\left({ }^{2} P\right) S d d^{3} D_{3}-\left({ }^{2} D\right) 4 f^{3} D_{2}$ \\
\hline 1 & & 3261.46 & 30652.21 & 1.72 & $\left({ }^{2} P\right) 6 s{ }^{3} P_{3}-\left({ }^{2} P\right) 6 p{ }^{3} S_{1}$ \\
\hline 5 & & 3257.85 & 30686.27 & 5.92 & $\left({ }^{2} D\right) 6 s^{1} D_{2}-\left({ }^{4} S\right) 4 f^{3} F_{2}$ \\
\hline 2 & & 3256.52 & 30698.80 & .84 & $\left({ }^{2} P\right) 5 d^{1} D_{2}-\left({ }^{2} D\right) 6 p{ }^{3} P_{2}$ \\
\hline 12 & & 3256.25 & 30701.35 & .25 & $-\left({ }^{2} P\right) 6 p{ }^{3} D_{3}$ \\
\hline 7 & & 3248.62 & 30773.45 & .36 & $-\left({ }^{2} D\right) 4 f^{3} P_{1}$ \\
\hline 22 & & 3246.85 & 30790.23 & .29 & $\left({ }^{2} D\right) 5 d d^{3} D_{3}-\left({ }^{4} S\right) 4 f^{3} F_{4}$ \\
\hline 12 & & 3244.13 & 30816.04 & .08 & $\left.{ }^{2} D\right) 6 p{ }^{1} D_{2}-\left({ }^{2} D\right) 6 d{ }^{\prime} P_{1}$ \\
\hline 25 & & 3242.86 & 30828.11 & .14 & $\left({ }^{4} S\right) S d^{3} D_{3}-\left({ }^{4} S\right) 6 p{ }^{3} P_{2}$ \\
\hline 10 & & 3240.47 & 30850.85 & .84 & $\left({ }^{2} P\right) 6 s{ }^{3} P_{2}-\left({ }^{2} D\right) 4 f^{1} F_{3}$ \\
\hline 25 & & 3236.84 & 30855.45 & .41 & $\left({ }^{4} S\right) S d^{3} D_{1}-\left({ }^{4} S\right) 6 p{ }^{3} P_{0}$ \\
\hline 13 & & 3235.73 & 30896.04 & 5.96 & $\left.{ }^{2} D\right) 4 f^{\prime} D_{2}$ \\
\hline 12 & & 3227.16 & 30978.08 & .08 & $\left.{ }^{4} S\right) 7 s{ }^{3} S_{4}$ \\
\hline 12 & & 3222.99 & 31018.16 & .12 & $-\left({ }^{2} D\right) 6 d d^{3} S_{1}$ \\
\hline 2 & & 3214.12 & 31103.76 & 2.98 & $-\left({ }^{2} P\right) 6 p{ }^{1} S_{0}$ \\
\hline 7 & & 3199.22 & 31248.62 & .66 & $\left.{ }^{2} D\right) 5 d{ }^{3} P_{1}-\left({ }^{2} P\right) 6 p{ }^{1} P_{1}$ \\
\hline 14 & & 3196.51 & 31275.11 & .07 & $\left({ }^{2} D\right) 5 d{ }^{1} P_{1}-\left({ }^{4} S\right) 6 p^{3} P_{1}$ \\
\hline 15 & & 3196.25 & 31277.65 & .64 & $\left.{ }^{2} D\right) 5 d^{3} D_{1}-\left({ }^{2} D\right) 6 p^{1} P_{1}$ \\
\hline 16 & & 3185.21 & 31386.06 & .22 & $\left.{ }^{2} D\right) 6 p{ }^{\prime} D_{2}-\left({ }^{2} D\right) 6 d^{\prime} D_{2}$ \\
\hline 11 & & 3184.27 & 31395.32 & .39 & $\left({ }^{2} D\right) 6 p^{1} P_{1}-\left({ }^{2} D\right) 7 s^{3} D_{1}$ \\
\hline 7 & & 3177.11 & 31466.07 & .02 & $\left({ }^{2} D\right) 6 p{ }^{1} P_{1}-\left({ }^{2} D\right) 6 d{ }^{3} F_{2}$ \\
\hline 7 & & 3169.75 & 31539.13 & .03 & $\left({ }^{2} D\right) 6 p{ }^{1} F_{3}-\left({ }^{2} D\right) 6 d^{3} F_{2}$ \\
\hline 9 & & 3164.47 & 31591.75 & .95 & $\left({ }^{2} D\right) 6 s{ }^{3} D_{3}-\left({ }^{4} S\right) 4 f^{3} F_{3}$ \\
\hline 8 & & 3160.66 & 31629.84 & .28 & $\left({ }^{2} D\right) 6 p \quad{ }^{\prime} P_{1}-\left({ }^{2} D\right) 7 s^{3} D_{2}$ \\
\hline 8 & & 3160.66 & 31629.84 & 8.83 & $-\left({ }^{2} P\right) 6 p{ }^{3} S_{1}$ \\
\hline 7 & & 3156.67 & 31669.81 & .85 & $\left({ }^{2} D\right) S d{ }^{3} D_{2}-\left({ }^{4} S\right) 4 f^{3} F_{2}$ \\
\hline 10 & & 3155.51 & 31681.46 & .51 & $\left({ }^{2} D\right) 5 d{ }^{3} P_{1}-\left({ }^{2} P\right) 6 D^{3} P_{2}$ \\
\hline 11 & & 3153.44 & 31702.25 & .29 & $\left({ }^{2} D\right) 6 p{ }^{\prime} F_{3}-\left({ }^{2} D\right) 7 s^{3} D_{2}$ \\
\hline 13 & & 3153.00 & 31706.67 & .64 & $\left({ }^{2} D\right) 6 S^{3} D_{2}-\left({ }^{4} S\right) 4{ }^{3}{ }^{5} F_{3}$ \\
\hline 15 & & 3151.83 & 31718.45 & .52 & $\left({ }^{2} P\right) S d{ }^{1} D_{2}-\left({ }^{2} D\right) 6 p{ }^{3} P_{1}$ \\
\hline 18 & . & 3150.97 & 31727.10 & 6.87 & $\left({ }^{2} D\right) 5 d^{1} G_{4}-\left({ }^{2} D\right) 6 p p^{\prime} F_{3}$ \\
\hline 14 & & 3150.83 & 31728.51 & .52 & $\left({ }^{4} S\right) 6 p{ }^{3} P_{2}-\left({ }^{4} S\right) 7 s{ }^{3} S_{1}$ \\
\hline is & & 3150.69 & 31729.92 & .90 & $\left({ }^{2} P\right) S d{ }^{3} P_{2}-\left({ }^{2} P\right) 6 P{ }^{3} S_{1}$ \\
\hline 11 & & 3141.63 & 31821.42 & .48 & $\left({ }^{4} S\right) S d d^{3} D_{2}-\left({ }^{4} S\right) 6 p^{3} P_{3}$ \\
\hline 20 & & 3138.28 & 31855.39 & .53 & $\left.{ }^{2} D\right) 6 p{ }^{\prime} D_{2}-\left({ }^{2} D\right) 6 d^{\prime} F_{3}$ \\
\hline 3 & & 3126.77 & 31972.65 & .77 & $\left({ }^{2} P\right) S d{ }^{3} P_{2}-\left({ }^{2} D\right) 4 f^{3} D_{3}$ \\
\hline 9 & & 3124.95 & 31991.27 & 0.52 & $\left({ }^{2} P\right) 6 s{ }^{3} P_{1}-\left({ }^{2} D\right) 4 f^{1} P_{1}$ \\
\hline 3 & & 3124.60 & 31994.85 & .83 & $\left({ }^{2} P\right) 5 d{ }^{3} D_{2}-\left({ }^{2} P\right) 6 p{ }^{1} P_{1}$ \\
\hline 9 & & 3122.15 & 32019.96 & .88 & $\left({ }^{2} P\right) 5 d{ }^{3} D_{3}-\left({ }^{2} D\right) 4 f^{3} P_{2}$ \\
\hline 5 & & 3121.01 & 32031.65 & .70 & $\left({ }^{2} D\right) 6 s^{3} D_{2}-\left({ }^{2} D\right) 6 p^{3} D_{3}$ \\
\hline 10 & & 3120.52 & 32036.68 & .78 & $\left({ }^{4} S\right) 6 p{ }^{3} P_{1}-\left({ }^{4} S\right) 6 d{ }^{5} D_{2}$ \\
\hline 12 & & 3114.41 & 32099.53 & .43 & $\left({ }^{2} D\right) 6 p \quad F_{3}-\left({ }^{2} D\right) 6 d^{\prime} G_{4}$ \\
\hline 6 & & 3107.56 & 32170.28 & .27 & $\left({ }^{2} D\right) 6 p \quad{ }^{1} F_{3}-\left({ }^{2} D\right) 6 d{ }^{3} F_{3}$ \\
\hline
\end{tabular}

Table I. Continued

\begin{tabular}{|c|c|c|c|c|c|}
\hline \multirow{2}{*}{\multicolumn{2}{|c|}{ Intensity' }} & \multirow[b]{2}{*}{$\lambda(A)$} & \multicolumn{2}{|l|}{$\sigma\left(\mathrm{cm}^{-1}\right)$} & \multirow[b]{2}{*}{ Classification } \\
\hline & & & obs & calc & \\
\hline 9 & & 3106.47 & 32181.57 & .64 & $\left({ }^{4} s\right) 6 p{ }^{3} P_{1}-\left({ }^{4} S\right) 7 s$ \\
\hline 13 & & 3106.34 & 32182.92 & .95 & $\left({ }^{2} D\right) 6 s s^{1} D_{2}-\left({ }^{2} P\right) 6 P$ \\
\hline 13 & & 3103.47 & 32212.68 & .67 & $\left({ }^{2} D\right)$ Gs ${ }^{3} D_{2}-\left({ }^{4} S\right) 4 f$ \\
\hline 10 & & 3102.69 & 32220.78 & .85 & $\left({ }^{4} s\right) 6 p$ \\
\hline 9 & & 3102.36 & 32224.21 & .03 & $\left({ }^{4} s\right) 4 f$ \\
\hline 11 & & 3099.87 & 32250.09 & .23 & $\left({ }^{4} S\right) 6 p^{3}{ }^{3} P_{1}-\left({ }^{4} S\right) 6 d$ \\
\hline 18 & & 3091.05 & 32342.11 & .15 & $\left({ }^{2} D\right) 5 d{ }^{3} G_{3}-\left({ }^{2} D\right) 6 p$ \\
\hline 11 & & 3090.00 & 32353.10 & .06 & $\left({ }^{2} D\right) 5 d d^{3} P_{1}-\left({ }^{2} D\right) 4 f$ \\
\hline 3 & & 3088.87 & 32364.93 & .97 & $\left({ }^{2} D\right) 6 p$ \\
\hline 18 & & 3083.53 & 32420.98 & 1.08 & $\left.{ }^{2} D\right) S_{d}{ }^{3} F_{4}-\left({ }^{2} D\right) 6 p$ \\
\hline 4 & b & 3082.89 & 32427.71 & .68 & $\left({ }^{2} P\right) 5 d{ }^{3} D_{2}-\left({ }^{2} P\right) 6 p$ \\
\hline 11 & & 3080.42 & 32453.71 & .72 & $\left({ }^{2} D\right) 6 p^{3} P_{1}-\left({ }^{2} D\right) 75$ \\
\hline 6 & & 3073.51 & 32526.67 & .67 & $\left({ }^{2} P\right) 6 s$ \\
\hline 2 & & 3068.57 & $32 \$ 79.03$ & $.2 \mathrm{I}$ & $(2 D) 6 p$ \\
\hline 18 & & 3065.19 & 32614.96 & 5.02 & $\left({ }^{2} P\right) S d$ \\
\hline 1 & & 3055.26 & 32720.95 & 1.06 & $\left({ }^{2} P\right) S d$ \\
\hline 14 & & 3054.48 & 32729.31 & .20 & $\left({ }^{2} P\right) S d$ \\
\hline 6 & & 3051.21 & 32764.39 & .76 & $\left({ }^{2} P\right) 6 p$ \\
\hline 7 & & 3048.86 & 32789.64 & .96 & $\left({ }^{2} P\right) 6 p$ \\
\hline 12 & & 3042.04 & 32863.15 & .36 & $\left({ }^{2} P\right) 6 p$ \\
\hline 12 & & 3026.52 & 33031.66 & .70 & $\left({ }^{2} D\right) S d$ \\
\hline 25 & & 3023.83 & 33061.04 & .01 & $(s) s d$ \\
\hline 18 & & 3023.65 & 33063.01 & .18 & $(S) 6 p$ \\
\hline 6 & & 3020.33 & 33099.35 & .23 & $\left({ }^{2} P\right) 5 d$ \\
\hline 2 & & 3015.77 & 33149.40 & .42 & $\left({ }^{2} P\right) 5 d$ \\
\hline 11 & & 3015.41 & 33153.36 & .36 & $(s) 4 r$ \\
\hline I1 & & 3014.59 & $33 \mathbf{1 6 2 . 3 8}$ & .43 & $\left({ }^{2} D\right) 6 p$ \\
\hline 15 & a & 3014.13 & 33167.44 & 6.89 & $\left({ }^{2} D\right) 5 d$ \\
\hline 1 & b & 3009.03 & 33223.65 & .57 & $\left.{ }^{2} D\right) 5 d^{3}$ \\
\hline 5 & & 3004.65 & 33272.08 & .11 & $\left({ }^{2} D\right) 5 d$ \\
\hline 18 & a & 3004.26 & 33276.40 & .31 & ('S)6p \\
\hline 11 & & 3001.52 & 33306.77 & .79 & $\left({ }^{4} S\right) 4 f$ \\
\hline 9 & & 2999.29 & 33331.54 & .62 & $\left({ }^{2} D\right) 6 s^{3}$ \\
\hline 11 & a & 2999.03 & 33334.43 & .33 & $\left({ }^{2} D\right) 6 p^{3}$ \\
\hline 9 & & 2997.50 & 33351.44 & .48 & $\left({ }^{2} D\right) 6 p$ \\
\hline 8 & & 2994.95 & 33379.84 & .92 & $\left.{ }^{4} s\right) 6 s$ \\
\hline 14 & & 2994.67 & 33382.96 & .86 & $(D) 6 p$ \\
\hline 4 & & 2994.37 & 33386.30 & .27 & $\left({ }^{2} P\right) 5 d$ \\
\hline 15 & & 2992.89 & 33402.81 & .91 & $(S) 6 p$ \\
\hline 11 & & 2991.49 & 33418.44 & .58 & $\left({ }^{2} D\right) 6 s$ \\
\hline 14 & & 2991.25 & 33421.12 & .17 & $\left({ }^{4} s\right) 6 p$ \\
\hline 3 & & 2990.82 & 33425.93 & 6.20 & $\left({ }^{2} D\right) 6 p$ \\
\hline 7 & & 2990.33 & 33431.40 & .39 & $\left({ }^{2} P\right) 5 d$ \\
\hline 11 & & 2986.11 & 33478.65 & .62 & $\left.{ }^{2} D\right) 6 p$ \\
\hline 14 & & 2985.53 & 33485.15 & .15 & $(s) 6 p$ \\
\hline 16 & & 2984.58 & 33495.81 & .77 & $\left({ }^{2} D\right) 6 p$ \\
\hline 8 & & 2981.32 & 33532.44 & .48 & $(D) 6 p$ \\
\hline 3 & & 2980.09 & 33546.27 & .12 & $\left({ }^{2} D\right) 6 p$ \\
\hline 3 & & 2980.09 & 33546.27 & .27 & $\left({ }^{2} P\right)$ Sd \\
\hline 10 & & 2976.75 & 33583.91 & .90 & $\left({ }^{2} D\right) 6 p$ \\
\hline 6 & b & 2974.90 & 33604.80 & .86 & $\left({ }^{2} P\right) S d^{3}$ \\
\hline 9 & & 2971.26 & 33645.96 & 6.07 & $\left.{ }^{2} D\right) 5 d^{3}$ \\
\hline 11 & & 2971.17 & 33646.98 & 7.07 & $\left.{ }^{2} D\right) 6 p$ \\
\hline 19 & & 2970.49 & 33654.69 & .77 & $(s) 6 p$ \\
\hline 10 & & 2970.08 & 33659.33 & .39 & $(' s) 4 f$ \\
\hline 10 & & 2970.08 & 33659.33 & .62 & $(s) 4 f$ \\
\hline 23 & & 2969.77 & 33662.84 & .28 & $\left({ }^{2} D\right) 5 d$ \\
\hline 9 & & 2969.43 & 33666.70 & .69 & $\left.{ }^{2} D\right) 6 p$ \\
\hline 12 & & 2968.57 & 33676.45 & .54 & $\left({ }^{4} S\right) 48$ \\
\hline 10 & & 2968.43 & 33678.04 & .18 & $(' s) 4 f$ \\
\hline 10 & $\mathbf{a}$ & 2966.92 & 33695.18 & .33 & $(s) 4 f$ \\
\hline 11 & & 2964.93 & 33717.79 & .70 & $\left({ }^{2} D\right) 6 p^{3}$ \\
\hline 13 & & 2964.17 & 33726.44 & .57 & $\left({ }^{2} D\right) 6 p^{3}$ \\
\hline 4 & & 2960.87 & 33764.03 & .02 & $\left({ }^{2} D\right) 5 d^{3}$ \\
\hline 13 & & 2960.34 & 33770.07 & .14 & \\
\hline 6 & & 2960.07 & 33773.15 & .19 & ( $D) S d$ \\
\hline 15 & $\mathbf{a}$ & 2959.36 & 33781.25 & 0.98 & $(s) 6 p^{3}$ \\
\hline 9 & $\mathbf{a}$ & 2959.28 & 33782.17 & .14 & ( DD) $5 d$ \\
\hline
\end{tabular}


Table I. Continued

Table I. Continued

$\sigma\left(\mathrm{cm}^{-1}\right)$

\begin{tabular}{cllll} 
Intensity $^{3}$ & $\lambda(\AA)$ & obs & \multicolumn{1}{l}{ calc } & \multicolumn{1}{l}{ Classification } \\
\hline 17 & 2954.93 & 33831.90 & 2.31 & $\left({ }^{2} D\right) 5 d^{3} D_{1}-\left({ }^{2} D\right) 6 p^{3} P_{2}$
\end{tabular}

$\begin{array}{llllrl}17 & & 2954.93 & 33831.90 & 2.31 & \left({ }^{2} D\right) 5 d d^{3} D_{1}-\left({ }^{2} D\right) 6 p{ }^{3} P_{2} \\ 10 & \text { a } & 2954.17 & 33840.60 & .60 & \left({ }^{2} D\right) 6 p{ }^{3} D_{1}-\left({ }^{2} D\right) 75\end{array}$

10 a $2954 \quad 33841.40 \quad .60$ (D)6P ${ }^{3} D_{3}-\left({ }^{2} D\right) 7 s^{1} D_{2}$

$\begin{array}{llllll}10 & \text { a } & 2954.10 & 33841.40 & .49 & \left({ }^{2} P\right) 5 d{ }^{3} F_{4}-\left({ }^{2} D\right) 4 f^{3} D_{3} \\ 7 & & 2953.94 & 33843.23 & .05 & \left({ }^{2} D\right) 5 d d^{\prime} G_{4}-\left({ }^{2} D\right) 6 p{ }^{3} F_{4}\end{array}$

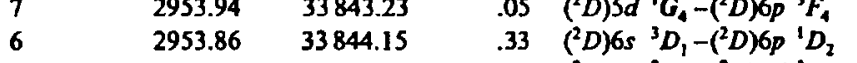

$3 \quad 2951.53 \quad 33870.87 \quad .86 \quad(D) 6 p{ }^{3} F_{1}-\left({ }^{2} D\right) 6 d{ }^{3} F_{4}$

$19 \quad 2948.07 \quad 33910.62 \quad 31 \quad .81 \quad\left({ }^{2} D\right) 5 d^{3} G_{3}-\left({ }^{2} D\right) 6 p{ }^{3} D_{2}$

$17 \quad 2947.51 \quad 33917.06 \quad .00 \quad\left({ }^{2} D\right) 6 p{ }^{3} F_{4}-\left({ }^{2} D\right) 6 d^{3} G_{5}$

$18 \quad 2945.23 \quad 33943.32 \quad 26 \quad .26 \quad\left({ }^{2} D\right) 6 p{ }^{3} F_{3}-\left({ }^{2} D\right) 6 d^{\prime} G_{4}$

$\begin{array}{lllll}10 & 2944.68 & 33949.66 & .69 & (2 D) 6 p \\ & 3 P_{1}-\left({ }^{2} D\right) 6 d^{3} P_{1}\end{array}$

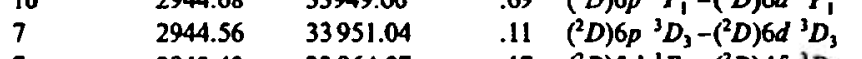

$7 \quad 2943.43 \quad 33964.07 \quad .17 \quad(D) 5 d^{\prime} F_{3}-\left({ }^{2} D\right) 4 f^{3} D_{3}$

\begin{tabular}{|c|c|}
\hline & l b $\quad 2942.06$ \\
\hline
\end{tabular}

$\begin{array}{llll}11 & \text { b } & 2942.06 & 33979.89 \\ 12 & & 2941.39 & 33987.63\end{array}$

$\begin{array}{lll}17 & 2940.21 & 34001.27 \\ 10 & 2939.73 & 34006.82\end{array}$

$\begin{array}{llll}12 & & 2939.73 & 34006.82 \\ 15 & \text { b } & 2939.11 & 34013.99 \\ 10 & & 2935.86 & 34051.64\end{array}$

$\begin{array}{llll}15 & \text { b } & 2935.86 & 34051.64 \\ 10 & & 2935.72 & 34053.27 \\ 18 & & 2932.76 & 34087.63\end{array}$

$\begin{array}{llll}10 & \text { b } & 2932.09 & 34095.42\end{array}$

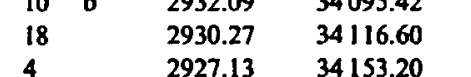

$\begin{array}{lll}4 & 2927.13 & 34153.20 \\ 11 & 2926.07 & 34165.57\end{array}$

$\begin{array}{lll}11 & 2926.07 & 34165.57 \\ 11 & 2923.96 & 34190.22\end{array}$

$\begin{array}{lll}16 & 2923.53 & 34195.25 \\ 15 & 2917.60 & 34264.75\end{array}$

$\begin{array}{lll}15 & 2917.60 & 34264.75 \\ 8 & 2916.63 & 34276.14 \\ 7 & 2915.04 & 34294.84\end{array}$

$\begin{array}{lll}7 & 2915.04 & 34294.84 \\ 18 & 2914.14 & 34305.43\end{array}$

$\begin{array}{lll}7 & 2914.14 & 34305.43 \\ 20 & 2912.38 & 34326.16\end{array}$

$\begin{array}{lll}19 & 2911.91 & 34331.70 \\ 6 & 2911.48 & 34336.77\end{array}$

$\begin{array}{lll}6 & 2911.48 & 34336.77 \\ 12 & 2910.37 & 34349.86\end{array}$

$\begin{array}{lll}12 & 2910.37 & 34349.86 \\ 3 & 2910.09 & 34353.17 \\ 15 & 2906.56 & 34394.89\end{array}$

$\begin{array}{lll}15 & 2906.56 & 34394.89 \\ 7 & 2904.17 & 34423.19\end{array}$

$\begin{array}{lll}7 & 2904.17 & 34423.19 \\ 5 & 2902.29 & 34445.49 \\ 4 & 2897.70 & 34500.05\end{array}$

$\begin{array}{lll}4 & 2897.70 & 34500.05 \\ 14 & 2896.65 & 34512.56\end{array}$

$\begin{array}{lll}14 & 2896.65 & 34512.56 \\ 2 & 2896.07 & 34519.47 \\ 6 & 2895.04 & 34531.75\end{array}$

$\begin{array}{lll}6 & 2895.04 & 34531.75 \\ 18 & 2891.72 & 34571.39\end{array}$

$\begin{array}{lll}18 & 2891.72 & 34571.39 \\ 8 & 2889.97 & 34592.33 \\ 2 & 2886.93 & 34628.75\end{array}$

$\begin{array}{lll}2 & 2886.93 & 34628.75 \\ 12 & 2886.68 & 34631.75\end{array}$

$\begin{array}{lll}12 & 2886.68 & 34631.75 \\ 5 & 2879.36 & 34719.79\end{array}$

\begin{tabular}{lll}
5 & 2879.36 & 34719.79 \\
4 & 2873.29 & 34793.13 \\
\hline & 2872.75 & 34799.67
\end{tabular}

$\begin{array}{lll}4 & 2872.75 & 34799.67 \\ 25 & 2871.69 & 34812.52\end{array}$

$\begin{array}{lll}25 & 2871.69 & 34812.52 \\ 25 & 2871.69 & 34812.52\end{array}$

$\begin{array}{lll}25 & 2871.69 & 34812.52 \\ 17 & 2871.27 & 34817.61 \\ 17 & 2871.10 & 34819.67 \\ 3 & 2869.52 & 34838.84\end{array}$

$\begin{array}{lll}3 & 2871.10 & 34819.67 \\ 3 & 2869.52 & 34838.84 \\ 8 & 2868.45 & 34851.84\end{array}$

$\begin{array}{lll}3 & 2868.45 & 34851.84 \\ 8 & 2864.62 & 34898.43 \\ 2 & 2863.86 & 34907.69\end{array}$

$\begin{array}{lll}2 & 2863.86 & 34907.69 \\ 25 & 2862.42 & 34925.25 \\ 3 & 2856.67 & 34995.55\end{array}$

\begin{tabular}{lll}
3 & 2856.67 & 34995.55 \\
4 & 2850.27 & 35074.12 \\
\hline & 2847.92 & 35103.06
\end{tabular}

$\begin{array}{lll}4 & 2850.27 & 35074.12 \\ 10 & 2847.92 & 35103.06\end{array}$

$\begin{array}{lll}14 & 2847.67 & 35106.15 \\ 7 & 2845.06 & 35138.35\end{array}$

$\begin{array}{lll}7 & 2845.06 & 35138.35 \\ 4 & 2844.12 & 35149.96 \\ 5 & 2838.81 & 35215.71\end{array}$

$\begin{array}{lll}5 & 2838.81 & 35215.71 \\ 8 & 2833.12 & 35286.43 \\ 6 & 2832.97 & 35288.30\end{array}$

$\begin{array}{lll}6 & 2832.97 & 35288.30 \\ 2 & 2832.83 & 35290.04\end{array}$

Physica Scripla 38

(D) $5 d d^{\prime} G_{4}-\left({ }^{2} D\right) 6 P^{3} D_{3}$

$.53 \quad\left({ }^{2} D\right) 6 p{ }^{3} D_{2}-\left({ }^{2} D\right) 6 d{ }^{3} G_{3}$

$.88 \quad\left({ }^{2} D\right) 5 d^{3} P_{2}-\left({ }^{2} D\right) 4 f^{3} D_{3}$

$4.10 \quad\left({ }^{2} D\right) 6 p{ }^{3} F_{3}-\left({ }^{2} D\right) 6 d{ }^{3} F_{3}$

$.62\left({ }^{4} S\right) 4 f f^{3} F_{3}-\left({ }^{2} D\right) 6 d{ }^{3} F_{4}$

$.33 \quad\left({ }^{2} D\right) 6 p{ }^{1} F_{3}-\left({ }^{2} D\right) 6 d^{3} P_{2}$

.53 ( $\left.{ }^{4} S\right) 5 d{ }^{3} D_{0}-\left({ }^{4} S\right) 6 p{ }^{5} P_{1}$

$.40 \quad\left({ }^{2} D\right) 6 p{ }^{3} F_{4}-\left({ }^{2} D\right) 6 d{ }^{3} D_{3}$

$.56 \quad\left({ }^{4} S\right) 4 f^{3} F_{4}-\left({ }^{2} D\right) 6 d{ }^{3} G_{3}$

$.15 \quad\left({ }^{2} D\right) 5 d^{3} P_{1}-\left({ }^{2} D\right) 4 f^{3} D_{1}$

$.65 \quad\left({ }^{4} S\right) 4 f^{5} F_{3}-\left({ }^{2} D\right) 7 s^{1} D_{2}$

$.21 \quad P P) 5 d{ }^{3} P_{2}-\left({ }^{2} P\right) 6 p{ }^{3} D_{3}$

$.33\left({ }^{2} D\right) 5 d{ }^{3} G_{5}-\left({ }^{4} S\right) 4 f{ }^{s} F_{4}$

.91 $\left({ }^{2} D\right) 5 d^{3} F_{4}-\left({ }^{2} D\right) 6 p{ }^{1} F_{3}$

$.17 \quad\left({ }^{4} S\right) 4 f{ }^{3} F_{3}-\left({ }^{2} D\right) 6 d{ }^{3} D_{3}$

$.96\left({ }^{4} S\right) 4 f^{3} F_{4}-\left({ }^{2} D\right) 6 d d^{3} D_{1}$

$.66\left({ }^{2} D\right) 5 d{ }^{3} F_{2}-\left({ }^{2} D\right) 6 p{ }^{3} D_{1}$

.31 ( $\left.{ }^{4} S\right) 6 p{ }^{3} P_{2}-\left({ }^{4} S\right) 6 d^{3} D_{3}$

$.58 \quad\left({ }^{4} S\right) 5 d d^{5} D_{1}-\left({ }^{4} S\right) 6 p{ }^{5} P_{1}$

$.73 \quad\left({ }^{2} D\right) 5 d{ }^{3} S_{1}-\left({ }^{2} P\right) 6 p{ }^{3} S_{1}$

$8.94 \quad\left({ }^{2} D\right) 6 p{ }^{3} D_{2}-\left({ }^{2} D\right) 6 d{ }^{3} F_{3}$

.07 ( $\left.{ }^{2} P\right) 5 d d^{\prime} F_{3}-\left({ }^{4} S\right) S f^{3} F_{4}$

$.88 \quad\left({ }^{2} D\right) 5 d^{3} G_{5}-\left({ }^{2} D\right) 6 p{ }^{3} F_{4}$

.16 ( $\left.{ }^{2} P\right) 5 d^{3} D_{1}-\left({ }^{2} D\right) 4 f^{1} D_{2}$

$.88 \quad\left({ }^{2} D\right) 6 p{ }^{3} P_{2}-\left({ }^{2} D\right) 6 d^{3} D_{2}$

$.22 \quad\left({ }^{2} P\right) 5 d{ }^{3} P_{1}-\left({ }^{2} P\right) 6 p{ }^{3} D_{1}$

$.52\left({ }^{4} S\right) 5 d{ }^{3} D_{1}-\left({ }^{4} S\right) 6 p{ }^{5} P_{2}$

$.36 \quad\left({ }^{2} P\right) 5 d{ }^{3} D_{2}-\left({ }^{2} D\right) 4 f^{3} P_{2}$

$\left..42 \quad{ }^{4} S\right) 6 p{ }^{3} P_{2}-\left({ }^{4} S\right) 6 d^{3} D_{1}$

$.53 \quad\left({ }^{2} D\right) 5 d{ }^{3} F_{3}-\left({ }^{2} D\right) 6 p^{3} F_{2}$

$.47 \quad\left({ }^{2} D\right) 6 p P^{1} P_{1}-\left({ }^{2} D\right) 6 d^{3} S_{1}$

$.32\left({ }^{2} P\right) 5 d d^{1} F_{3}-\left({ }^{4} S\right) 5 f^{5} F_{3}$

$2.11\left({ }^{4} S\right) 4 \int^{5} F_{2}-\left({ }^{2} D\right) 6 d{ }^{3} D_{2}$

$.91 \quad\left({ }^{2} D\right) 6 p{ }^{3} P_{1}-\left({ }^{2} D\right) 6 d d^{1} P_{1}$

.37 (2P) $5 d{ }^{3} P_{0}-\left({ }^{2} P\right) 6 p{ }^{3} D_{1}$

$\left..69 \quad{ }^{2} D\right) 5 d{ }^{3} D_{1}-\left({ }^{2} P\right) 6 p{ }^{3} D_{2}$

.68 ( $\left.{ }^{2} D\right) 5 d d^{3} G_{4}-\left({ }^{2} D\right) 6 p{ }^{3} F_{3}$

$3.08 \quad\left({ }^{2} D\right) 6{ }^{3} D_{3}-\left({ }^{2} D\right) 6 d^{3} D_{2}$

$.64\left({ }^{4} S\right) 5 d d^{3} D_{2}-\left({ }^{4} S\right) 6 p{ }^{3} P_{2}$

80 (' $S) 6 p{ }^{3} P_{1}-\left({ }^{4} S\right) 6 d^{3} D_{2}$

$.45 \quad\left({ }^{2} P\right) 6 s{ }^{3} P_{1}-\left({ }^{2} P\right) 6 p{ }^{3} P_{2}$

.98 ( $\left.{ }^{2} D\right) 5 d^{3} D_{1}-\left({ }^{2} D\right) 6 p^{3} P_{1}$

9.32 ( $\left.{ }^{2} P\right) S d{ }^{3} D_{2}-\left({ }^{2} D\right) 4 \int^{3} D_{1}$

.73 ( $\left.{ }^{2} D\right) 6 s{ }^{1} D_{2}-\left({ }^{2} P\right) 6 p{ }^{3} D_{2}$

.10 ('S)Sd ${ }^{3} D_{2}-\left({ }^{4} S\right) 6 p{ }^{5} P_{1}$

.79 ('P)Sd $1 F_{3}-\left({ }^{2} P\right) 4 f^{3} G_{3}$

$.24\left({ }^{4} S\right) 6 s^{3} S_{1}-\left({ }^{2} D\right) 6 p{ }^{3} F_{2}$

$2.82\left({ }^{2} D\right) 5 d^{3} P_{2}-\left({ }^{2} D\right) 4 f^{\prime} P_{1}$

.04 ( $S$ C $S d{ }^{5} D_{2}-\left({ }^{4} S\right) 6 p{ }^{5} P_{2}$

.14 ('S)4f ${ }^{5} F_{3}-\left({ }^{2} D\right) 6 d{ }^{3} D_{2}$

$.84 \quad\left({ }^{2} D\right) 5 d^{3} D_{3}-\left({ }^{2} D\right) 4 f^{3} G_{3}$

$.74 \quad\left({ }^{2} D\right) 6 p{ }^{3} F_{2}-\left({ }^{2} D\right) 7 s^{3} D_{1}$

.37 ( $\left.{ }^{2} D\right) 6 p{ }^{3} F_{2}-\left({ }^{2} D\right) 6 d{ }^{3} F_{2}$

$.33 \quad\left({ }^{2} D\right) 6 s^{3} D_{3}-\left({ }^{4} S\right) 4 f{ }^{3} F_{4}$

.05 $\quad\left({ }^{2} D\right) 6 p^{3} P_{1}-\left({ }^{2} D\right) 6 d d^{1} D_{2}$

\begin{tabular}{|c|c|c|c|c|c|}
\hline & & & $\sigma\left(\mathrm{cm}^{-1}\right)$ & & \\
\hline Intensity & & $\lambda(\AA)$ & obs & calc & Classification \\
\hline 17 & & 2827.46 & 35357.06 & .01 & $\left({ }^{4} S\right) 5 d{ }^{5} D_{3}-\left({ }^{4} S\right) 6 p{ }^{5} P_{2}$ \\
\hline 14 & & 2825.99 & 35375.45 & .46 & $\left({ }^{(} S\right) 6 p{ }^{5} P_{2}-\left({ }^{4} S\right) 6 d^{5} D_{2}$ \\
\hline 8 & b & 2817.36 & 35483.81 & .79 & $\left({ }^{2} P\right){ }^{3} d{ }^{3} P_{2}-\left({ }^{2} P\right) 6 p{ }^{1} P_{1}$ \\
\hline 16 & & 2815.92 & 35501.95 & 2.06 & $\left({ }^{4} S\right) 6 p{ }^{3} P_{2}-\left({ }^{4} S\right) 6 d d^{s} D_{3}$ \\
\hline 1 & & 2815.27 & 35510.15 & .00 & $\left({ }^{2} P\right) 6 s{ }^{3} P_{1}-\left({ }^{2} D\right) 4 f$ \\
\hline 13 & a & 2814.46 & 35520.37 & .32 & $\left({ }^{4} S\right) 6 p p^{5} P_{2}-\left({ }^{4} S\right) 7 s$ \\
\hline 8 & & 2811.61 & 35556.37 & .40 & $\left({ }^{4} S\right) 6 p{ }^{3} P_{1}-\left({ }^{4} S\right) 6 d d^{5}$ \\
\hline 7 & b & 2810.46 & 35570.92 & .20 & $\left({ }^{2} D\right) 6 p{ }^{3} F_{2}-\left({ }^{2} D\right) 6 d^{3} G_{1}$ \\
\hline 3 & & 2809.53 & 35582.69 & .74 & $\left({ }^{2} D\right) 65^{3} D_{2}-\left({ }^{4} S\right) 4 f^{3} F_{3}$ \\
\hline 10 & $\mathbf{a}$ & 2809.06 & 35588.65 & .62 & $\left({ }^{2} D\right) 6 s^{3} D_{1}-\left({ }^{4} S\right) 4 f^{3}$ \\
\hline 10 & a & 2809.06 & 35588.65 & .90 & $\left({ }^{4} S\right) 6 p{ }^{3} P_{2}-\left({ }^{4} S\right) 6 d^{3}$ \\
\hline 7 & & 2808.57 & 35594.86 & .80 & $\left({ }^{2} D\right) 6 p{ }^{1} F_{3}-\left({ }^{2} D\right) 7 s^{3}$ \\
\hline 7 & & 2808.46 & 35596.25 & .26 & $\left({ }^{2} D\right) 5 d d^{\prime} F_{3}-\left({ }^{2} P\right) 6 p$ \\
\hline 12 & & 2807.22 & 35611.97 & .95 & $\left({ }^{2} D\right) 6 p p^{1} F_{3}-\left({ }^{2} D\right) 6 d^{3}$ \\
\hline 6 & & 2806.40 & 35622.38 & .35 & ( $P$ P) $5 d d^{1} D_{2}-\left({ }^{2} D\right) 6 p$ \\
\hline 4 & & 2805.09 & 35639.01 & 8.97 & $\left({ }^{2} D\right) 5 d{ }^{3} P_{2}-\left({ }^{2} P\right) 6 p$ \\
\hline 3 & & 2802.19 & 35675.90 & .54 & $\left({ }^{2} D\right) 5 d{ }^{3} S_{1}-\left({ }^{2} D\right) 4 f$ \\
\hline 17 & & 2800.20 & 35701.25 & .26 & $\left({ }^{4} S\right) 6 p{ }^{5} P_{1}-\left({ }^{4} S\right) 7 s$ \\
\hline 9 & & 2797.87 & 35730.98 & .94 & $\left({ }^{2} D\right) 5 d^{3} D_{3}-\left({ }^{2} D\right) 4 f$ \\
\hline 12 & & 2797.12 & 35740.56 & .46 & ('S)6p ${ }^{5} P_{1}-(S S) 6 d$ \\
\hline 14 & & 2794.84 & 35769.71 & .84 & $-\left({ }^{4} S\right) 6 d$ \\
\hline 3 & & 2792.47 & 35800.07 & 1.02 & $\left.{ }^{2} D\right) 5 d ' D$ \\
\hline 7 & & 2785.80 & 35885.78 & .74 & $\left({ }^{2} P\right) 5 d^{3} \mathrm{~L}$ \\
\hline 6 & & 2785.33 & 35891.83 & .67 & $\left({ }^{2} D\right) 5 d^{3} D_{2}-\left({ }^{2} P\right) 6 p^{3}$ \\
\hline 7 & & 2784.93 & 35896.99 & 7.16 & $(D) 6 p{ }^{3} F_{3}-\left({ }^{2} D\right) 6 d d^{3}$ \\
\hline 17 & & 2783.33 & 35917.62 & .61 & $\left.{ }^{2} D\right) 6 p{ }^{3} F_{2}-\left({ }^{2} D\right) 6 d$ \\
\hline 10 & & 2782.69 & 35925.89 & .81 & $-\left({ }^{2} D\right) 6 d$ \\
\hline 4 & & 2782.30 & 35930.92 & .87 & ( $P) 5 d{ }^{3} D_{2}-\left({ }^{2} D\right) 4 f$ \\
\hline 11 & & 2779.64 & 35965.30 & .07 & $2-\left({ }^{2} P\right) 6 p^{3}$ \\
\hline 13 & & 2777.94 & 35987.31 & .04 & $(2 D) 6 p p^{\prime} F_{3}-\left({ }^{2} D\right) 6 d$ \\
\hline 16 & & 2776.96 & 36000.01 & 5999.97 & $\left({ }^{2} D\right) 5 d{ }^{3} F_{2}-\left({ }^{2} D\right) 6 p^{3}$ \\
\hline 6 & a & 2774.79 & 36028.16 & .06 & $-\left({ }^{2} D\right) 75$ \\
\hline $\mathbf{0}$ & & 2774.44 & 36032.71 & .53 & $-\left({ }^{2} D\right) 4 f$ \\
\hline 16 & & 2772.40 & 36059.22 & 8.92 & $-\left({ }^{2} P\right) 6 p$ \\
\hline 16 & & 2772.40 & 36059.22 & .33 & $(s) 6 p^{5}$ \\
\hline 10 & & 2769.17 & 36101.28 & .07 & $-\left({ }^{2} D\right) 7 s$ \\
\hline 15 & & 2766.18 & 36140.30 & .20 & $\left({ }^{2} D\right) 5 d^{3} F_{3}-\left({ }^{2} D\right) 6 p$ \\
\hline 5 & & 2765.95 & 36143.30 & .31 & $\left({ }^{2} D\right) 5 d^{\prime} D_{2}-\left({ }^{2} P\right) 4 f$ \\
\hline 6 & & 2763.01 & 36181.76 & .53 & (2D)5d ${ }^{3} F_{4}-\left({ }^{4} S\right) 4 f$ \\
\hline 6 & & 2763.01 & 36181.76 & .61 & $\left.{ }^{2} D\right) 5 d^{\prime} F$ \\
\hline 9 & b & 2762.73 & 36185.43 & .33 & $\left({ }^{2} D\right) 6 p{ }^{3} F$ \\
\hline 15 & & 2761.58 & 36200.49 & .32 & $\left({ }^{2} D\right) 5 d^{3} \mathrm{~F}$ \\
\hline 13 & & 2760.72 & 36211.77 & .58 & $\left({ }^{2} D\right) 6 p$ ' \\
\hline 13 & & 2760.72 & 36211.77 & .69 & (2D)Sd ${ }^{3} s$ \\
\hline 11 & & 2759.19 & 36231.85 & 2.01 & $\left({ }^{2} D\right) 6 p$ \\
\hline 3 & & 2757.56 & 36253.26 & 2.79 & $\left({ }^{2} P\right) S d$ \\
\hline 13 & & 2754.88 & 36288.53 & .05 & ${ }^{4} S{ }^{4} 6 p^{3}{ }^{3}$ \\
\hline 14 & & 2747.86 & 36381.23 & .09 & $\left({ }^{2} D\right) 5 d^{3} I$ \\
\hline 16 & & 2740.78 & 36475.21 & .04 & $\left({ }^{2} D\right) 5 d{ }^{3} /$ \\
\hline 4 & & 2739.19 & 36496.38 & 5.95 & $(4 S) 4 f^{3} F$ \\
\hline 14 & & 2736.99 & 36525.71 & .38 & $\left({ }^{2} D\right) 5 d^{3} F$ \\
\hline 9 & & 2728.20 & 36643.39 & 2.91 & $\left({ }^{4} S\right) 6 s{ }^{3} S_{1}-\left({ }^{2} D\right) 6 p$ \\
\hline 11 & a & 2727.20 & 36656.83 & .51 & $\left({ }^{2} D\right) 5 d{ }^{3} G_{d}-\left({ }^{2} D\right) 6 p$ \\
\hline 1 & $w, b$ & 2725.69 & 36677.13 & 6.93 & $\left({ }^{2} D\right) 6 p{ }^{3} D_{3}-\left({ }^{2} D\right) 6 d$ \\
\hline 2 & & 2718.10 & 36779.54 & .03 & $\left({ }^{2} D\right) 6 p{ }^{3} P_{2}-\left({ }^{2} D\right) 6 d$ \\
\hline 2 & & 2714.05 & 36834.42 & .01 & $\left({ }^{2} P\right) S d{ }^{3} F$ \\
\hline 10 & a & 2713.39 & 36843.38 & 4.15 & $\left({ }^{2} D\right) 6 p^{3}$ \\
\hline 0 & & 2711.34 & 36871.24 & .22 & $\left({ }^{2} P\right) S d{ }^{3} F$ \\
\hline 6 & & 2710.94 & 36876.68 & 7.23 & $\left({ }^{2} D\right) 5 d d^{3}$ \\
\hline 12 & & 2708.45 & 36910.58 & .06 & $(2 D) 6 p^{3} I$ \\
\hline 3 & & 2704.41 & 36965.71 & .27 & $\left({ }^{4} S\right) 4 f^{3}$ \\
\hline 2 & & 2702.31 & 36994.44 & 3.90 & $\left({ }^{2} D\right) 5 d^{1} F_{3}-\left({ }^{2} D\right) 4 f$ \\
\hline $\mathbf{0}$ & & 2701.71 & 37002.66 & 1.98 & $\left({ }^{4} S\right) 4 f^{5} F_{3}$ \\
\hline 5 & & 2701.50 & 37005.53 & 4.76 & $(s) 6 p{ }^{3} P_{1}-\left({ }^{4} S\right) 7 s^{3}$ \\
\hline 15 & b & 2696.51 & 37074.01 & .23 & $\left({ }^{4} S\right) 5 d d^{3} D_{1}-\left({ }^{2} D\right) 6 p$ \\
\hline 15 & b & 2696.51 & 37074.01 & 3.55 & $20 \mathrm{~L}=0$ \\
\hline 3 & & 2695.65 & 37085.83 & .18 & \\
\hline 3 & & 2695.65 & 37085.83 & .66 & $\left({ }^{2} D\right) 6 s{ }^{3} D$ \\
\hline
\end{tabular}


Table I. Continued

\begin{tabular}{|c|c|c|c|c|c|c|c|c|c|c|c|}
\hline \multirow{2}{*}{\multicolumn{2}{|c|}{ Intensity' }} & \multirow[b]{2}{*}{$\lambda(A)$} & \multicolumn{2}{|l|}{$\sigma\left(\mathrm{cm}^{-1}\right)$} & \multirow[b]{2}{*}{ Classification } & \multirow{2}{*}{\multicolumn{2}{|c|}{ Intensity' }} & \multirow[b]{2}{*}{$\lambda(\mathcal{A})$} & \multicolumn{2}{|l|}{$\sigma\left(\mathrm{cm}^{-1}\right)$} & \multirow[b]{2}{*}{ Classification } \\
\hline & & & obs & calc & & & & & obs & calc & \\
\hline 5 & & 2694.12 & 37106.89 & .82 & $\left.{ }^{2} D\right) 6 p{ }^{1} P_{1}-\left({ }^{2} D\right) 6 d{ }^{3} P_{0}$ & 10 & & 2447.083 & 40852.60 & .86 & $\left({ }^{2} D\right) 5 d{ }^{3} D_{3}-\left({ }^{2} P\right) 6 p$ \\
\hline 12 & $\mathbf{a}$ & 2691.44 & 37143.84 & .95 & $\left.{ }^{2} D\right) 6 p{ }^{3} D_{1}-\left({ }^{2} D\right) 7 s^{3} D_{2}$ & 8 & & 2446.503 & 40862.29 & .77 & $\left({ }^{2} P\right) S d{ }^{3} P_{1}-\left({ }^{2} D\right) 4 f$ \\
\hline 4 & & 2691.22 & 37146.88 & .24 & $\left({ }^{2} D\right) 6 p{ }^{3} D_{3}-\left({ }^{2} D\right) 6 d^{1} F_{3}$ & 11 & & 2441.523 & 40945.63 & 6.55 & $\left({ }^{2} D\right) S d{ }^{3} F_{3}-\left({ }^{2} D\right) 6 p$ \\
\hline 5 & & 2685.51 & 37225.86 & .00 & $\left({ }^{2} P\right) S d{ }^{3} P_{1}-\left({ }^{2} P\right) 6 p{ }^{3} D_{2}$ & 6 & $w$ & 2439.516 & 40979.32 & .97 & $\left({ }^{2} P\right) 5 d^{3} F_{3}-\left({ }^{2} P\right) 6 p$ \\
\hline 6 & & 2678.55 & 37322.58 & .41 & $\left({ }^{2} D\right) 6 s s^{3} D_{2}-\left({ }^{2} D\right) 6 p{ }^{1} D_{2}$ & 12 & & 2436.491 & 41030.18 & .39 & $\left({ }^{4} S\right) 5 d^{3} D_{3}-\left({ }^{2} D\right) 6 p$ \\
\hline 6 & & 2678.55 & 37322.58 & .47 & $\left({ }^{4} S\right) 6 p^{5} P_{3}-\left({ }^{4} S\right) 6 d^{3} D_{3}$ & 3 & & 2423.918 & 41243.00 & .42 & $\left({ }^{2} P\right) 5 d d^{3} F_{4}-\left({ }^{2} D\right) 4 f$ \\
\hline 6 & & 2670.25 & 37438.58 & .64 & $\left({ }^{2} D\right) 6 p^{3} F_{3}-\left({ }^{2} D\right) 7 s^{3} D_{3}$ & 9 & & 2422.767 & 41262.59 & 3.03 & $\left({ }^{4} S\right) 6 s{ }^{3} S_{1}-\left({ }^{4} S\right) 4 f$ \\
\hline 20 & & 2669.01 & 37455.98 & .79 & $\left({ }^{2} D\right) 6 p{ }^{3} F_{3}-\left({ }^{2} D\right) 6 d^{3} G_{4}$ & 14 & b & 2422.139 & 41273.29 & .35 & $\left({ }^{2} P\right) 5 d{ }^{3} F_{3}-\left({ }^{2} D\right) 4 f$ \\
\hline 20 & & 2669.01 & 37455.98 & 6.16 & $\left({ }^{4} S\right) 5 d{ }^{5} D_{3}-\left({ }^{4} S\right) 6 p{ }^{5} P_{3}$ & 7 & & 2419.740 & 41314.20 & .13 & $\left({ }^{2} P\right) 5 d{ }^{3} F_{2}-\left({ }^{2} D\right) 4 f$ \\
\hline 10 & $\mathbf{a}$ & 2667.94 & 37471.00 & .30 & $\left({ }^{4} S\right) 4 f^{3} F_{3}-\left({ }^{2} D\right) 6 d^{1} F_{3}$ & 8 & & 2418.744 & 41331.22 & .33 & $\left({ }^{2} D\right) 5 d{ }^{3} P_{2}-\left({ }^{2} D\right) 4 f$ \\
\hline 7 & & 2661.00 & 37568.72 & .64 & $\left({ }^{2} D\right) 5 d d^{3} F_{2}-\left({ }^{2} D\right) 6 p{ }^{3} D_{2}$ & 12 & & 2416.744 & 41365.41 & .23 & $\left({ }^{4} S\right) 5 d d^{3} D_{3}-\left({ }^{2} D\right) 6 p$ \\
\hline 5 & . & 2659.36 & 37591.88 & .55 & $\left({ }^{2} P\right) 5 d{ }^{3} P_{0}-\left({ }^{2} P\right) 6 p{ }^{3} P_{1}$ & 9 & & 2414.544 & 41403.10 & .21 & $\left({ }^{2} P\right) 5 d{ }^{3} P_{1}-\left({ }^{2} P\right) 6 p$ \\
\hline 9 & & 2658.27 & 37607.30 & .15 & $\left({ }^{4} S\right) S d{ }^{5} D_{0}-\left({ }^{4} S\right) 6 p{ }^{3} P_{1}$ & 4 & & 2414.230 & 41408.49 & .68 & $\left({ }^{2} D\right) 5 d d^{3} D_{2}-\left({ }^{2} D\right) 4 f$ \\
\hline 2 & & 2641.13 & 37851.34 & .20 & $\left({ }^{4} S\right) 5 d{ }^{5} D_{1}-\left({ }^{4} S\right) 6 p{ }^{3} P_{1}$ & 4 & & 2414.230 & 41408.49 & .82 & $\left({ }^{2} D\right) 5 d{ }^{3} P_{2}-\left({ }^{2} D\right) 4 f$ \\
\hline 9 & & 2639.15 & 37879.74 & .64 & $\left.{ }^{2} D\right) 6 p{ }^{3} D_{1}-\left({ }^{2} D\right) 6 d^{3} D_{1}$ & 2 & & 2414.104 & 41410.64 & 1.23 & $\left({ }^{2} D\right) S d{ }^{1} F_{3}-\left({ }^{2} D\right) 4 f$ \\
\hline 13 & & 2634.21 & 37950.77 & .75 & $\left({ }^{2} D\right) S d{ }^{3} P_{2}-\left({ }^{2} P\right) 6 p{ }^{3} P_{2}$ & 6 & & 2412.505 & 41438.10 & .21 & $\left({ }^{2} D\right) S d{ }^{3} D_{3}-\left({ }^{2} P\right) 6 p$ \\
\hline 7 & & 2626.98 & 38055.21 & .41 & ( $D) 6 p{ }^{3} F_{3}-\left({ }^{2} D\right) 6 d^{3} D_{3}$ & 1 & & 2406.229 & 41546.16 & .25 & $\left({ }^{2} D\right) 6 s{ }^{1} D_{2}-\left({ }^{2} P\right) 6 P$ \\
\hline 4 & & 2624.52 & 38090.88 & .62 & $\left.{ }^{2} D\right) 5 d d^{3} S_{1}-\left({ }^{2} P\right) 6 p{ }^{1} P_{1}$ & 9 & & 2403.792 & 41588.28 & .45 & $\left({ }^{2} P S d{ }^{1} D_{2}-\left({ }^{2} P\right) 6 p\right.$ \\
\hline 8 & $\mathbf{w}$ & 2619.85 & 38158.77 & .48 & $\left({ }^{4} S\right) 6 p{ }^{5} P_{2}-\left({ }^{4} S\right) 6 d{ }^{3} D_{2}$ & 8 & & 2397.566 & 41696.27 & .36 & $\left({ }^{2} P\right) 5 d{ }^{3} P_{0}-\left({ }^{2} P\right) 6 p$ \\
\hline 0 & & 2616.90 & 38201.79 & .42 & $\left({ }^{2} D\right) 5 d d^{1} F_{3}-\left({ }^{2} D\right) 4 f^{3} F_{3}$ & 10 & & 2394.083 & 41756.92 & .90 & $\left({ }^{4} S\right) S d{ }^{3} D_{2}-\left({ }^{2} D\right) 6 p$ \\
\hline 1 & & 2616.63 & 38205.73 & .67 & $\left({ }^{2} D\right) 5 d d^{3} G_{3}-\left({ }^{2} D\right) 6 p{ }^{3} F_{4}$ & 4 & b & 2392.376 & 41786.71 & .59 & $\left({ }^{2} D\right) 5 d{ }^{3} G_{5}-\left({ }^{4} S\right) 4 f$ \\
\hline 1 & & 2613.99 & 38244.31 & .13 & $\left({ }^{2} D\right) 5 d d^{3} P_{2}-\left({ }^{2} D\right) 4 f^{3} F_{3}$ & 11 & & 2388.573 & 41853.25 & .35 & $\left({ }^{2} P\right) 5 d{ }^{3} D_{1}-\left({ }^{2} P\right) 4 f$ \\
\hline 1 & & 2611.03 & 38287.67 & .21 & $\left({ }^{2} P\right) 6 s{ }^{3} P_{0}-\left({ }^{2} D\right) 4 f^{3} D_{1}$ & 8 & b & 2385.902 & 41900.09 & 1.00 & $\left({ }^{2} D\right) 5 d^{3} G_{3}-\left({ }^{4} S\right) 4 f$ \\
\hline 5 & & 2610.57 & 38294.41 & .25 & $\left({ }^{2} D\right) 6 p{ }^{1} P_{1}-\left({ }^{2} D\right) 6 d^{1} P_{1}$ & 11 & & 2385.673 & 41904.11 & .05 & $\left({ }^{2} D\right) 5 d{ }^{3} S_{1}-\left({ }^{2} D\right) 4 f$ \\
\hline 7 & b & 2607.50 & 38339.50 & .42 & $\left.{ }^{4} S\right) 6 p{ }^{3} P_{1}-\left({ }^{4} S\right) 6 d^{3} D_{2}$ & 13 & & 2383.947 & 41946.77 & .90 & $\left({ }^{2} P\right) 5 d \quad{ }^{1} F_{3}-\left({ }^{2} P\right) 4 f$ \\
\hline 15 & $\mathbf{a}$ & 2600.12 & 38448.31 & 7.72 & $5 s 5 p^{5} \quad{ }^{3} P_{0}-\left({ }^{4} S\right) 6 p p^{3} P_{1}$ & 11 & & 2382.087 & 41967.19 & .34 & $5 s 5 p^{5} \quad{ }^{3} P_{0}-\left({ }^{4} s\right) 6 p$ \\
\hline 7 & $\mathbf{a}$ & 2595.03 & 38523.72 & .47 & $\left({ }^{2} D\right) S d{ }^{3} S_{1}-\left({ }^{2} P\right) 6 p{ }^{3} P_{2}$ & 3 & & 2379.783 & 42007.82 & .79 & $\left.{ }^{2} D\right) 5 d{ }^{3} F$ \\
\hline 7 & & 2594.53 & 38531.14 & 0.93 & $\left({ }^{2} D\right) S d{ }^{3} G_{3}-\left({ }^{4} S\right) 4 f^{5} F_{2}$ & 0 & & 2378.714 & 42026.69 & .65 & $\left({ }^{2} D\right) S d{ }^{3} S_{1}-\left({ }^{2} D\right) 4 f$ \\
\hline 10 & & 2591.69 & 38573.36 & .13 & $\left({ }^{2} D\right) 5 d^{3} G_{4}-\left({ }^{4} S\right) 4 f^{5} F$ & 18 & b & 2369.595 & 42188.42 & .76 & $\left({ }^{2} D\right) S d{ }^{3} F_{2}-\left({ }^{2} S\right) 4 f$ \\
\hline 8 & & 2591.24 & 38580.06 & 79.59 & $\left.{ }^{(2} D\right) 5 d^{1} F_{3}-\left({ }^{2} D\right) 4 f^{3} D_{2}$ & 12 & & 2366.115 & 42250.46 & .50 & $\left({ }^{2} D\right) 5 d{ }^{3} D_{3}-\left({ }^{2} D\right) 4 f$ \\
\hline 12 & & 2590.42 & 38592.27 & 1.92 & $\left({ }^{2} D\right) S d d^{3} G_{4}-\left({ }^{4} S\right) 4 f^{5} F_{3}$ & 14 & & 2365.416 & 42262.94 & 3.01 & $\left({ }^{2} P\right) 5 d{ }^{3} D_{2}-\left({ }^{2} P\right) 4 f$ \\
\hline 9 & & 2578.62 & 38768.86 & .55 & $\left({ }^{4} S\right) 5 d^{3} D_{1}-\left({ }^{2} D\right) 6 p{ }^{3} F_{2}$ & 0 & & 2359.159 & 42375.02 & .00 & $\left({ }^{2} D\right) 5 d{ }^{3} F_{2}-\left({ }^{2} D\right) 6 p{ }^{3} P_{2}$ \\
\hline 13 & & 2578.37 & 38772.62 & .69 & $\left({ }^{2} D\right) 5 d^{3} G_{4}-\left({ }^{2} D\right) 6 p{ }^{3} F_{4}$ & 11 & & 2354.456 & 42459.67 & .48 & $\left({ }^{2} P\right) 5 d{ }^{3} D_{1}-\left({ }^{4} S\right) S f$ \\
\hline 2 & & 2574.61 & 38829.24 & .47 & $\left.{ }^{2} D\right) 6 p{ }^{1} D_{2}-\left({ }^{2} P\right) 6 d{ }^{3} D_{1}$ & 8 & b & 2353.936 & 42469.04 & 8.93 & $\left({ }^{4} S\right) 6 s{ }^{3} S$ \\
\hline 7 & & 2572.33 & 38863.66 & .68 & $\left({ }^{2} P\right) S d d^{1} D_{2}-\left({ }^{2} P\right) 6 p^{3} D_{1}$ & 8 & & 2350.563 & 42529.98 & 30.18 & $\left.{ }^{2} D\right) 5 d^{3} D_{2}-\left({ }^{2} P\right) 6 p$ \\
\hline 7 & & 2572.33 & 38863.66 & 4.39 & $\left.{ }^{2} D\right) 6 p P^{1} P_{1}-\left({ }^{2} D\right) 6 d d^{1} D_{2}$ & 1 & & 2339.398 & 42732.94 & 3.30 & $\left({ }^{2} D\right) 6 p{ }^{3} P_{1}-\left({ }^{2} P\right) 6 d$ \\
\hline 5 & & 2570.26 & 38894.96 & .59 & $\left({ }^{4} S\right) 6 s^{3} S_{1}-\left({ }^{2} D\right) 6 p{ }^{1} P_{1}$ & 9 & & 2338.884 & 42742.34 & .02 & $\left({ }^{2} P\right) 5 d{ }^{3} P_{1}$ \\
\hline 9 & & 2568.81 & 38916.91 & .98 & $\left({ }^{2} D\right) S d{ }^{3} G_{4}-\left({ }^{2} D\right) 6 p{ }^{3} D_{3}$ & 7 & & 2333.555 & 42839.94 & .83 & $\left.{ }^{2} D\right) 6 s{ }^{\prime} D_{2}-\left({ }^{2} P\right) 6 p p^{\prime} /$ \\
\hline 9 & & 2568.81 & 38916.91 & 7.39 & $\left({ }^{2} D\right) 6 p{ }^{3} F_{3}-\left({ }^{2} D\right) 6 d^{3} D_{2}$ & 0 & & 2326.369 & 42972.26 & 1.63 & $\left({ }^{2} D\right) 5 d d^{3} P_{1}-\left({ }^{4} S\right) S f$ \\
\hline 3 & & 2550.55 & 39195.51 & .02 & $\left({ }^{2} D\right) 5 d^{3} S_{1}-\left({ }^{2} D\right) 4 f^{3} D_{2}$ & 3 & & 2323.455 & 43026.14 & .74 & $\left.\left({ }^{2} D\right) 6 s^{3} D_{3}-\left({ }^{2} D\right) 4 f^{3}\right]$ \\
\hline 2 & & 544.10 & 39294.87 & .32 & $\left({ }^{2} P\right) 5 d d^{3} F_{2}-\left({ }^{2} P\right) 6 p p^{3} D_{3}$ & 5 & & 2320.992 & 43071.79 & .65 & $\left.\left({ }^{2} P\right) 5 d d^{3} F_{3}-\left({ }^{2} D\right) 4 f^{3}\right)$ \\
\hline 3 & & 2541.88 & 39329.19 & 8.81 & $\left({ }^{2} D\right) 6 s \quad D_{2}-\left({ }^{2} D\right) 4 f^{3} D_{3}$ & 10 & & 2316.583 & 43153.76 & 4.06 & $\left({ }^{2} D\right) 6 p^{3} F$ \\
\hline 1 & & 2541.028 & 39342.34 & .15 & $\left({ }^{2} D\right) 6 p{ }^{3} F_{2}-\left({ }^{2} D\right) 7 s^{3} D_{3}$ & 12 & & 2313392 & 43213.29 & .28 & $\left({ }^{4} S\right) 6 p{ }^{3} P_{1}$ \\
\hline 1 & & 2538.918 & 39375.03 & 4.70 & $\left({ }^{2} P\right) S d{ }^{3} P_{2}-\left({ }^{2} D\right) 4 f^{1} F_{3}$ & 11 & & 2312.276 & 43234.14 & 3.94 & $\left.{ }^{2} D\right) 5 d{ }^{\prime} P_{1}$ \\
\hline 7 & & 2536.868 & 39406.85 & .71 & $\left({ }^{2} D\right) 6 p{ }^{1} F_{3}-\left({ }^{2} D\right) 6 d^{\prime} F_{3}$ & 7 & & 2309.922 & 43278.20 & .17 & $\left.{ }^{(2} P\right) 5 d{ }^{3} P_{1}-\left({ }^{2} P\right) 6 p \quad{ }^{1} D_{2}$ \\
\hline 7 & & 2533.318 & 39462.07 & 1.72 & $\left({ }^{4} S\right) 5 d d^{3} D_{3}-\left({ }^{2} D\right) 6 p^{3} F_{2}$ & 5 & & 2305.502 & 43361.15 & .05 & $\left({ }^{2} P\right) 5 d d^{3} D_{2}-\left({ }^{2} P\right) 4 f^{3}$ \\
\hline 7 & & 2523.967 & 39608.25 & 7.83 & $\left({ }^{4} S\right) S d d^{5} D_{1}-\left({ }^{4} S\right) 6 p{ }^{3} P_{2}$ & 5 & & 2305.502 & 43361.15 & .92 & $\left({ }^{2} D\right) 6 s^{3} D_{2}-\left({ }^{2} P\right) 6 p^{3}$ \\
\hline 5 & & 2521.428 & 39648.14 & 7.88 & $\left({ }^{2} D\right) 6 s{ }^{3} D_{3}-\left({ }^{2} D\right) 4 f^{3} G_{3}$ & 9 & & 2303.735 & 43394.42 & .21 & $5 s 5 p^{5} \quad{ }^{3} P_{1}$ \\
\hline 6 & & 2515.117 & 39747.61 & .32 & $\left({ }^{2} D\right) S d{ }^{3} F_{2}-\left({ }^{2} D\right) 6 p{ }^{\prime} F_{3}$ & 9 & & 2303.735 & 43394.42 & .67 & $\left({ }^{2} D\right) 5 d{ }^{3} F_{2}$ \\
\hline $\mathbf{0}$ & h & 2514.09 & 39763.89 & .53 & $\left({ }^{2} P\right) S d d^{3} D_{3}-\left({ }^{2} P\right) 4 \int^{3} F_{3}$ & 9 & & 2300.876 & 43448.33 & .21 & $(D D) 6 s{ }^{3} D_{1}$ \\
\hline 1 & & 2513.337 & 39775.76 & .71 & ( $\left.{ }^{2} D\right) 6 p{ }^{3} D_{2}-\left({ }^{2} D\right) 6 d^{3} P_{1}$ & 9 & & 2300.716 & 43451.35 & .22 & $\left({ }^{(} S\right) 5 d d^{3} D_{2}-\left({ }^{2} D\right) 6 p{ }^{3} F_{2}$ \\
\hline 1 & & 2511.288 & 39808.22 & 7.66 & $\left({ }^{4} S\right) 6 p p^{5} P_{1}-\left({ }^{4} S\right) 6 d^{3} D_{1}$ & 9 & & 2300.353 & 43458.22 & .02 & $\left({ }^{2} D\right) 5 d d^{3} D_{3}-\left({ }^{2} D\right) 4 f{ }^{3} F_{3}$ \\
\hline 4 & & 2509.74 & 39832.81 & .51 & $555 p^{5} \quad{ }^{1} P_{1}-\left({ }^{2} P\right) 4 f^{3} D_{2}$ & I & & 2298.540 & 43492.48 & .39 & $\left({ }^{2} P\right) S d{ }^{3} F_{2}-\left({ }^{2} D\right) 4 f^{3} D_{1}$ \\
\hline 11 & & 504.907 & 39909.61 & .04 & $\left.{ }^{2} D\right) 6 s{ }^{3} D_{1}-\left({ }^{2} P\right) 6 p{ }^{3} P_{0}$ & 12 & b & 2290.834 & 43638.77 & .67 & $\left.{ }^{2} D\right) 6 s^{3} D$ \\
\hline 12 & & 2501.037 & 39971.36 & 0.95 & $\left.{ }^{2} D\right) 5 d^{1} P_{1}-\left({ }^{2} D\right) 6 p^{3} D_{1}$ & 2 & & 2288.009 & 92.65 & .62 & $\left({ }^{2} P\right) 5 d{ }^{3} D_{2}$ \\
\hline 9 & & 2486.727 & 40201.37 & .34 & $\left({ }^{4} S\right) S d d^{5} D_{2}-\left({ }^{4} S\right) 6 p{ }^{3} P_{2}$ & 7 & & 2286.634 & 43718.91 & .81 & ( $\left.{ }^{2} D\right) 6 s^{3} D_{3}-\left({ }^{2} D\right) 4 f^{3} D_{3}$ \\
\hline 7 & & 2485.006 & 40229.20 & 8.98 & $\left.{ }^{2} D\right) 6 s^{3} D_{3}-\left({ }^{2} D\right) 4 f^{3} G_{4}$ & 5 & & 2283.828 & 43772.63 & .79 & $\left({ }^{2} D\right) 5 d{ }^{3} F_{4}-\left({ }^{4} S\right) 4 f{ }^{3} F_{4}$ \\
\hline 6 & & 2483.464 & 40254.18 & .29 & $\left({ }^{(} D\right) S d{ }^{3} F_{3}-\left({ }^{4} S\right) 4 f^{5} F_{3}$ & 1 & & 2273.709 & 43967.41 & .18 & $\left({ }^{2} P\right) 5 d^{3} D_{2}-\left({ }^{4} S\right) S f^{9} F_{2}$ \\
\hline 8 & & 2479.876 & 40312.42 & .74 & $\left({ }^{2} D\right) 5 d^{3} D_{2}-\left({ }^{2} D\right) 4 f^{3} D_{3}$ & 3 & & .2273 .488 & 43971.70 & .73 & ( $\left.{ }^{2} P\right) 6 s \quad{ }^{1} P_{1}-\left({ }^{2} P\right) 4 f^{3} D_{2}$ \\
\hline 7 & & 2479.130 & 40324.56 & .63 & $\left({ }^{4} S\right) 6 s s^{3} S_{1}-\left({ }^{2} D\right) 6 p^{3} P_{0}$ & 2 & & 2272.609 & 43988.70 & .65 & $\left({ }^{2} D\right) 6 s^{3} L$ \\
\hline $\mathbf{0}$ & & 2477.062 & 40358.21 & .27 & $\left({ }^{4} S\right) S d d^{5} D_{1}-\left({ }^{4} S\right) 6 p{ }^{3} P_{0}$ & 11 & $w$ & 2271.382 & 44012.46 & .37 & $\left.{ }^{2} D\right) 5 d d^{\prime} F_{3}$ \\
\hline 7 & & 2472.377 & 40434.68 & 5.05 & $\left({ }^{2} D\right) 5 d d^{3} F_{3}-\left({ }^{2} D\right) 6 p{ }^{3} F_{4}$ & 8 & & 2271.055 & 44018.80 & .94 & $\left({ }^{4} S\right) 5 d{ }^{3} D_{1}-\left({ }^{2} D\right) 6 p{ }^{3}$ \\
\hline 10 & & 2471.319 & 40452.00 & .31 & $\left({ }^{4} S\right) 5 d{ }^{5} D_{3}-\left({ }^{4} S\right) 6 p^{3} P_{2}$ & 4 & & 2268.923 & 44060.15 & .09 & $\left({ }^{2} P\right) 5 d{ }^{3} D_{2}-\left({ }^{2} P\right) 4 f^{3} C$ \\
\hline 7 & b & 2468.393 & 40499.94 & 500.10 & $\left({ }^{2} D\right) S d d^{3} D_{1}-\left({ }^{4} S\right) 4 \int^{3}{ }^{3} F_{2}$ & 5 & & 2258.847 & 44256.68 & .62 & $\left({ }^{2} D\right) 5 d d^{3} D_{2}-\left({ }^{2} P\right) 6 p{ }^{3} P_{2}$ \\
\hline 2 & & 2464.555 & 40563.01 & .73 & ( $\left.{ }^{2} D\right) 6{ }^{3} D_{2}-\left({ }^{2} P\right) 6 p{ }^{3} D_{1}$ & 0 & & 2249.630 & 44437.97 & 8.04 & $\left({ }^{2} P\right) S d^{3} F_{3}-\left({ }^{2} D\right) 4 f^{\prime} F_{3}$ \\
\hline 10 & & 2463.557 & 40579.44 & .34 & ( $\left.{ }^{2} D\right) 5 d^{3} F_{3}-\left({ }^{2} D\right) 6 p^{3} D_{3}$ & 6 & & 2247.556 & 44478.99 & .82 & $\left({ }^{2} P\right) S d d^{3} F_{2}-\left({ }^{2} D\right) 4 f^{1} F_{3}$ \\
\hline 10 & & 2463.061 & 40587.61 & .90 & $\left({ }^{2} P\right) 5 d{ }^{3} F_{2}-\left({ }^{2} P\right) 6 p \quad{ }^{1} P_{1}$ & 5 & & 2247.335 & 44483.36 & .16 & $\left({ }^{2} P\right) 5 d d^{3} F_{3}-\left({ }^{2} D\right) 4 r^{1}$ \\
\hline 11 & & 2452.644 & 40759.98 & 60.32 & $\left({ }^{2} D\right) 5 d{ }^{3} F_{3}-\left({ }^{4} S\right) 4 \int^{3} F_{2}$ & 8 & & 2245.280 & 44524.07 & 3.94 & $\left({ }^{2} P\right) 5 d d^{3} F_{2}-\left({ }^{2} D\right) 4 f^{1}$ \\
\hline
\end{tabular}

Table I. Continued 
Table I. Continued

\begin{tabular}{|c|c|c|c|c|c|c|c|c|c|}
\hline \multirow{2}{*}{ Intensity } & \multirow[b]{2}{*}{$\lambda(\mathrm{A})$} & \multicolumn{2}{|l|}{$\sigma\left(\mathrm{cm}^{-1}\right)$} & \multirow{2}{*}{ Classification } & \multirow[b]{2}{*}{ Intensity ${ }^{\mathrm{j}}$} & \multirow[b]{2}{*}{$\lambda(\mathrm{A})$} & \multicolumn{2}{|l|}{$\sigma\left(\mathrm{cm}^{-1}\right)$} & \multirow[b]{2}{*}{ Classification } \\
\hline & & obs & calc & & & & obs & calc & \\
\hline 11 & 2243.970 & 44550.06 & 49.99 & $\left({ }^{2} D\right) 5 d d^{3} D_{2}-\left({ }^{2} D\right) 4 \int^{3} F_{3}$ & 2 & 2043.286 & 48925.07 & .15 & $\left({ }^{2} P\right) S d{ }^{3} F_{4}-\left({ }^{4} S\right) 5 f{ }^{5} F_{3}$ \\
\hline 2 & 2235.349 & 44721.86 & .92 & $\left({ }^{2} D\right) S d d^{3} D_{1}-\left({ }^{2} P\right) 6 p{ }^{3} D_{2}$ & 1 & 2041.276 & 48973.24 & .17 & $\left({ }^{2} D\right) 5 d d^{1} G_{4}-\left({ }^{2} D\right) 4 f^{3} H_{4}$ \\
\hline 7 & 2231.673 & 44795.52 & .32 & $\left({ }^{2} D\right) 5 d{ }^{3} D_{1}-\left({ }^{2} P\right) 6 p{ }^{3} P_{1}$ & 7 & 2039.314 & 49020.36 & .57 & $\left({ }^{4} S\right) 5 d^{3} D_{1}-\left({ }^{4} S\right) 4 f^{3} F_{3}$ \\
\hline 6 & 2230.419 & 44820.69 & .52 & $\left({ }^{2} D\right) 5 d d^{3} D_{1}-\left({ }^{2} P\right) 6 p{ }^{3} P_{0}$ & 6 & 2038.394 & 49042.48 & .79 & $\left({ }^{2} D\right) 5 d d^{3} F_{2}-\left({ }^{4} S\right) 4 f{ }^{3} F_{2}$ \\
\hline 3 & 2227.588 & 44877.64 & .60 & $\left({ }^{2} D\right) 6 p{ }^{3} P_{0}-\left({ }^{2} P\right) 6 d^{3} D_{1}$ & 7 & 2037.670 & 49059.89 & .97 & $\left({ }^{2} D\right) 5 d{ }^{1} P_{1}-\left({ }^{2} D\right) 6 p^{3}$ \\
\hline 9 & 2225.071 & 44928.41 & .16 & ( $\left.{ }^{2} D\right) 5 d{ }^{3} D_{2}-\left({ }^{2} D\right) 4 d^{3} D_{2}$ & 8 & 2031.996 & 49196.86 & .86 & $\left({ }^{2} D\right) 5 d d^{3} G_{5}-\left({ }^{2} D\right) 4 f^{3} H_{5}$ \\
\hline 5 & 2223.640 & 44957.33 & .34 & $\left({ }^{C} S\right) S d{ }^{3} D_{1}-\left({ }^{4} S\right) \mathcal{A} f^{S} F_{2}$ & 9 & 2029.216 & 49264.25 & 3.67 & $\left({ }^{2} P\right) 5 d^{3} P_{0}-\left({ }^{2} D\right) 4 f^{3} P_{1}$ \\
\hline 2 & 2220.544 & 45019.99 & .89 & $\left({ }^{4} S\right) 5 d{ }^{3} D_{2}-\left({ }^{2} D\right) 6 p{ }^{3} D_{2}$ & 8 & 2029.023 & 49268.94 & .97 & $\left({ }^{2} D\right) 5 d^{3} D_{3}-\left({ }^{2} D\right) 4 f^{1} G_{4}$ \\
\hline 9 & 2214.382 & 45145.26 & 4.48 & $\left({ }^{4} S\right) S d{ }^{3} D_{3}-\left({ }^{4} S\right) 4 f^{3} F_{3}$ & 10 & 2026.062 & 49340.94 & 1.68 & $\left({ }^{2} D\right) 6 s^{3} D_{2}-\left({ }^{2} P\right) 6 p{ }^{\prime} D_{2}$ \\
\hline 0 & 2213799 & 45157.14 & .10 & $\left({ }^{2} P\right) 5 d d^{3} P_{1}-\left({ }^{P} P\right) 6 p{ }^{1} P_{1}$ & 2 & 2021.304 & 49457.06 & 6.84 & $\left({ }^{2} D\right) 5 d d^{3} S_{1}-\left({ }^{2} P\right) 4 f^{3} F_{2}$ \\
\hline 9 & 2210.420 & 45226.17 & .22 & $\left.{ }^{(} P\right) 5 d^{1} D_{2}-\left({ }^{2} D\right) 4 f^{3} F_{2}$ & 6 & 2020.914 & 49466.60 & .71 & $\left({ }^{2} P\right) 5 d^{3} D_{2}-\left({ }^{2} P\right) 4 f^{3}$ \\
\hline 3 & 2205.587 & 45325.27 & .24 & $\left({ }^{4} S\right) 5 d d^{3} D_{3}-\left({ }^{2} D\right) 6 p{ }^{3} F_{4}$ & 5 & 2018.714 & 49520.52 & .55 & $\left({ }^{2} P\right) 5 d^{\prime} D_{2}-\left({ }^{2} P\right) 6 p p^{\prime}$ \\
\hline 5 & 2204.333 & 45351.04 & 0.90 & $\left({ }^{2} D\right) 6 s^{3} D_{3}-\left({ }^{2} P\right) 6 p{ }^{1} D_{2}$ & 2 & 2017.911 & 49540.22 & .45 & $\left({ }^{2} D\right) 5 d d^{1} F_{3}-\left({ }^{2} P\right) 4 \int^{3} G_{3}$ \\
\hline 0 & 2203.682 & 45364.44 & .36 & $\left({ }^{2} D\right) 6 s^{1} D_{2}-\left({ }^{2} D\right) 4 f^{3} P_{2}$ & 2 & 2016.174 & 49582.89 & 3.16 & $\left({ }^{2} D\right) 5 d{ }^{3} P_{2}-\left({ }^{2} P\right) 4 f{ }^{3} G_{3}$ \\
\hline 7 & 2202.695 & 45384.76 & .96 & $\left({ }^{2} D\right) 5 d d^{3} G_{3}-\left({ }^{4} S\right) 4 f^{3} F_{2}$ & 8 & 2013.859 & 49639.88 & 40.00 & $\left({ }^{4} S\right) S d d^{3} D_{2}-\left({ }^{4} S\right) 4 \int^{3} F_{2}$ \\
\hline 6 & 2199.514 & 45450.39 & .25 & $\left({ }^{2} P\right) 5 d d^{3} P_{0}-\left({ }^{2} P\right) 6 p{ }^{1} P_{1}$ & 4 & 2006.317 & 49826.46 & .24 & $\left({ }^{4} S\right) 5 d^{3} D_{2}-\left({ }^{2} D\right) 6 p^{3} P_{2}$ \\
\hline 6 & 2199.287 & 45455.09 & 4.77 & $\left({ }^{2} P\right) 6 s{ }^{3} P_{2}-\left({ }^{2} P\right) 4 f^{3} D_{3}$ & I & 2001.085 & 49956.71 & .93 & $\left({ }^{2} D\right) S d d^{3} G_{3}-\left({ }^{2} D\right) 4 f^{3} G_{3}$ \\
\hline 1 & 2198.583 & 45469.64 & .53 & $\left({ }^{4} S\right) 5 d d^{3} D_{3}-\left({ }^{2} D\right) 6 p^{3} D_{3}$ & 0 & 1997.497 & 50062.65 & .97 & (CD) $5 d{ }^{3} S_{1}-\left({ }^{4} S\right) 5 \int^{\mathrm{s}} F_{2}$ \\
\hline 10 & 2197.801 & 45485.82 & .63 & $\left.{ }^{2} D\right) 5 d^{1} P_{1}-\left({ }^{2} D\right) 6 p{ }^{1} P_{1}$ & 6 & 1997.330 & 50066.83 & 7.07 & $\left({ }^{4} S\right) 5 d^{3} D_{1}-\left({ }^{2} D\right) 6 p{ }^{1} D_{2}$ \\
\hline 1 & 2194.292 & 45558.56 & .83 & $\left({ }^{2} D\right) 5 d d^{3} F_{2}-\left({ }^{4} S\right) 4 f^{3} F_{3}$ & 7 & 1990.182 & 50246.66 & .78 & $\left({ }^{2} P\right) S d d^{1} D_{2}-\left({ }^{2} D\right) 4 f^{3} F_{3}$ \\
\hline 1 & 2192.428 & 45597.28 & .37 & $\left({ }^{2} D\right) 5 d^{3} G_{3}-\left({ }^{4} S\right) 4 f^{3} F_{4}$ & 1 & 1989.377 & 50267.00 & .07 & $\left({ }^{2} D\right) 6 s^{3} D_{1}-\left({ }^{2} D\right) 4 f{ }^{3} P_{2}$ \\
\hline 12 & 2189.828 & 45651.42 & 0.51 & $\left({ }^{4} S\right) S d d^{3} D_{3}-\left({ }^{4} S\right) 4 f^{5} F_{2}$ & 10 & 1985.405 & 50367.56 & .32 & $\left({ }^{2} P\right) S d d^{3} D_{3}-\left({ }^{2} P\right) 4 \int^{3} F_{4}$ \\
\hline 3 & 2185.701 & 45751.90 & .97 & $\left({ }^{2} P\right) S d d^{3} P_{2}-\left({ }^{2} P\right) 4 f^{3} F_{3}$ & 5 & 1983.985 & 50403.61 & .59 & $\left({ }^{4} S\right) 5 d{ }^{5} D_{2}-\left({ }^{2} D\right) 6 p^{3}$ \\
\hline 7 & 2179.684 & 45863.84 & .61 & $\left({ }^{2} D\right) 6 s^{3} D_{1}-\left({ }^{2} P\right) 6 p D^{1} D_{2}$ & 2 & 1980.606 & 50489.61 & .67 & $\left({ }^{2} P\right) 5 d^{3} D_{2}-\left({ }^{2} P\right) 4 f^{3}$ \\
\hline 9 & 2172.779 & 46009.58 & .53 & $\left({ }^{P} P\right) 5 d \quad d^{\prime} D_{2}-\left({ }^{2} D\right) 4 f^{3} D_{3}$ & 2 & 1979.266 & 50523.78 & .95 & $\left({ }^{2} D\right) 5 d{ }^{3} G_{4}-\left({ }^{2} D\right) 4 f^{3} G_{3}$ \\
\hline 3 & 2165.526 & 46163.67 & .24 & $\left({ }^{4} S\right) 5 d{ }^{3} D_{1}-\left({ }^{2} D\right) 6 p^{3} P_{1}$ & 8 & 1978.705 & 50538.10 & .02 & $\left({ }^{2} D\right) 5 d{ }^{3} G_{3}-\left({ }^{2} D\right) 4 f \cdot{ }^{3} G_{4}$ \\
\hline 3 & 2165.526 & 46163.67 & 4.39 & $\left({ }^{2} D\right) 5 d d^{3} G_{4}-\left({ }^{5} S\right) 4 f^{3} F_{4}$ & 1 & 1974.445 & 50647.15 & .02 & $\left({ }^{2} D\right) 6 s{ }^{3} D_{1}-\left({ }^{2} D\right) 4 f^{3} D_{1}$ \\
\hline 7 & 2160.935 & 46261.74 & .50 & (2P) $5 d d^{3} P_{1}-\left({ }^{2} D\right) 4 f^{3} D_{2}$ & 4 & 1973.829 & 50662.96 & 3.22 & $5 s 5 p^{5} \quad{ }^{3} P_{0}-\left({ }^{2} D\right) 6 p{ }^{3} D_{1}$ \\
\hline 8 & 2156.896 & 46348.36 & .29 & $\left({ }^{2} D\right) 5 d^{3} D_{2}-\left({ }^{2} D\right) 4 f^{3} P_{2}$ & 1 & 1970.899 & 50738.26 & .43 & $\left({ }^{4} S\right) S d{ }^{5} D_{2}-\left({ }^{2} D\right) 6 p{ }^{3} F_{3}$ \\
\hline 7 & 2147.683 & 46547.15 & .08 & $\left({ }^{4} S\right) 5 d^{3} D_{1}-\left({ }^{2} D\right) 6 p^{3} D_{1}$ & 7 & 1970.059 & 50759.90 & 60.24 & $\left({ }^{4} S\right) 5 d^{3} D_{3}-\left({ }^{2} D\right) 6 p p^{1} D_{2}$ \\
\hline o & 2144.189 & 46623.00 & 2.71 & $\left({ }^{2} D\right) 5 d^{3} D_{3}-\left({ }^{2} D\right) 4 f^{1} F_{3}$ & 4 & 1969.474 & 50774.98 & 5.09 & ( $\left.{ }^{2} D\right) 5 d d^{3} D_{1}-\left({ }^{2} P\right) 6 p \quad{ }^{1} D_{2}$ \\
\hline 2 & 2142.129 & 46667.82 & .83 & $\left({ }^{2} D\right) 5 d^{3} D_{3}-\left({ }^{2} D\right) 4 f^{1} D_{2}$ & 6 & 1967.905 & 50815.46 & .30 & $\left({ }^{2} P\right) 5 d{ }^{3} F_{3}-\left({ }^{2} P\right) 4 f^{3} F_{3}$ \\
\hline 7 & 2139.375 & 46727.90 & .23 & $\left({ }^{2} D\right) S d d^{3} G_{5}-\left({ }^{2} D\right) 4 f^{3} G_{4}$ & 1 & 1966.733 & 50845.75 & .91 & $\left({ }^{4} S\right) 5 d{ }^{3} D_{2}-\left({ }^{2} D\right) 6 p^{3} P_{1}$ \\
\hline 7 & 2139.375 & 46727.90 & 8.25 & $\left.{ }^{2} D\right) 5 d d^{3} D_{2}-\left({ }^{2} D\right) 4 f^{3} D_{1}$ & 7 & 1966.345 & 50855.78 & 6.08 & $\left({ }^{2} P\right) S d d^{3} F_{2}-\left({ }^{2} P\right) A f^{3} F_{3}$ \\
\hline 6 & 2139.236 & 46730.93 & .74 & $\left({ }^{2} D\right) 6 s^{\prime} D_{2}-\left({ }^{2} D\right) 4 f^{\prime} F_{3}$ & 7 & 1956.759 & 51104.92 & 5.04 & $\left.\left({ }^{2} D\right) S d{ }^{3} G_{4}-\left({ }^{2} D\right) 4\right)^{3}$ \\
\hline 9 & 2138.432 & 46748.50 & .54 & $\left({ }^{2} D\right) 6 s^{3} D_{3}-\left({ }^{2} D\right) 4 f^{3} F_{4}$ & 2 & 1956.154 & S1 120.73 & .75 & $\left({ }^{2} D\right) 6 s^{3} D_{3}-\left({ }^{2} D\right) 4 f^{\prime}$ \\
\hline 3 & 2130.809 & 46915.73 & .66 & $\left({ }^{2} D\right) S d^{1} P_{1}-\left({ }^{2} D\right) 6 p^{3} P_{0}$ & 1 & 1945.419 & 51402.81 & .75 & $\left({ }^{2} D\right) 5 d^{\prime} G_{4}-\left({ }^{2} D\right) 4 f^{\prime}$ \\
\hline 1 & 2128.473 & 46967.20 & .23 & $\left({ }^{2} P\right) 5 d d^{3} D_{3}-\left({ }^{(} P\right) 4 f^{3} D_{2}$ & 12 & 1942.913 & 51469.11 & .31 & $\left({ }^{2} P\right) 6 s^{1} /$ \\
\hline 4 & 2123.163 & 47084.65 & .30 & $\left({ }^{2} P\right) 5 d d^{3} F_{3}-\left({ }^{2} D\right) 4 f^{1} G_{4}$ & 4 & 1941.329 & 51511.11 & .21 & $\left({ }^{2} D\right) 5 d d^{3} F_{4}-\left({ }^{2} D\right) 4 f^{3} H_{4}$ \\
\hline 2 & 2122.205 & 47105.90 & .47 & $\left.{ }^{(P P}\right) 5 d \quad{ }^{\prime} D_{2}-\left({ }^{2} D\right) d f^{\prime} P_{1}$ & 6 & 1935.038 & 51678.58 & .57 & $\left.{ }^{2} D\right) 6 s^{3} L$ \\
\hline 9 & 2118.036 & 47198.62 & .56 & $\left({ }^{4} S\right) 5 d^{3} D_{2}-\left({ }^{2} D\right) 6 p{ }^{\prime} F_{3}$ & 11 & 1930.083 & 51811.25 & .36 & $\left({ }^{4} S\right) S d{ }^{3} D_{1}-\left({ }^{4} S\right) 4 \int^{3} F_{2}$ \\
\hline 7 & 2114.762 & 47271.68 & .57 & $\left({ }^{4} S\right) 5 d d^{3} D_{2}-\left({ }^{2} D\right) 6 p{ }^{1} P_{1}$ & 6 & 1929.158 & 51836.09 & .16 & $\left({ }^{2} D\right) 5 d^{3} F_{3}-\left({ }^{2} P\right) 6 p{ }^{3} D_{2}$ \\
\hline 1 & 2106.354 & 47456.31 & .14 & $\left({ }^{2} P\right) 5 d{ }^{3} P_{2}-\left({ }^{4} S\right) 5 f^{5} F_{2}$ & 5 & 1927.622 & 51877.40 & .48 & $\left({ }^{2} P\right) 6 s^{3}$ \\
\hline 0 & 2099.545 & 47614.26 & .34 & $\left({ }^{2} D\right) 5 d^{3} F_{3}-\left({ }^{4} S\right) 4 f^{3}$ & 2 & 1926.291 & 51913.24 & .34 & $\left({ }^{2} P\right) 5 d{ }^{3} F_{3}-\left({ }^{2} P\right) 4 f{ }^{3} F_{2}$ \\
\hline 9 & 2096.570 & 47681.81 & .63 & $\left({ }^{2} P\right) 5 d d^{3} P_{1}$ & 4 & 1924.757 & 51954.60 & .12 & $\left.\left({ }^{2} P\right) 5 d d^{3}\right)$ \\
\hline 3 & 2095.350 & 47709.56 & .59 & $\left({ }^{2} D\right) 6 s{ }^{3} D_{2}-\left({ }^{2} D\right) 4 f^{3} D_{3}$ & 4 & 1924.757 & 51954.60 & .59 & $\left({ }^{2} D\right) 5 d^{3}$ \\
\hline 6 & 2095.126 & 47714.67 & 68 & $\left({ }^{2} D\right) 5 d^{3} D_{2}-\left({ }^{2} D\right) 4 f{ }^{1} F_{3}$ & 8 & 1924.197 & 51969.74 & .66 & $\left({ }^{2} P\right) 5 d{ }^{3} F_{3}-\left({ }^{4} S\right) 5 f$ \\
\hline 5 & 2093.862 & 47743.47 & .37 & $\left({ }^{2} D\right) 5 d^{3} F_{3}-\left({ }^{2} P\right) 4 f^{3} F_{3}$ & 5 & 1921.644 & 52038.77 & .63 & $5 s 5 p^{5} \quad{ }^{3} P_{2}-\left({ }^{4} S\right) 6 p{ }^{3}$ \\
\hline 9 & 2091.996 & 47786.06 & .08 & $\left({ }^{2} D\right) 5 d{ }^{3} P_{2}-\left({ }^{2} P\right) 4 f^{3} F_{3}$ & 5 & 1916.211 & 52186.31 & .31 & $\left({ }^{2} D\right) S d^{3} F_{3}-\left({ }^{2} D\right) 4 f^{3} G_{3}$ \\
\hline 7 & 2090.225 & 47826.52 & .76 & $\left({ }^{2} D\right) 5 d d^{3} F_{3}-\left({ }^{4} S\right) 4 f^{3} F_{4}$ & 2 & 1915.595 & 52203.09 & .27 & $\left.\left({ }^{2} D\right) 5 d^{3} F_{4}-\left({ }^{2} D\right) 4\right)^{3}$ \\
\hline 9 & 2084.598 & 47955.61 & 6.06 & $\left({ }^{2} D\right) 6{ }^{3} D_{3}-\left({ }^{2} D\right) 4 f^{3} F_{3}$ & 2 & 1912.564 & 52285.82 & .69 & $\left({ }^{2} P\right) 5 d$ \\
\hline 2 & 2084.461 & 47958.77 & 9.01 & $\left({ }^{2} P\right) 5 d d^{3} D_{1}-\left({ }^{2} P\right) 4 f^{3} D_{2}$ & 0 & 1911.131 & $\$ 2325.03$ & .01 & $\left({ }^{2} D\right) 6 s^{3} D$ \\
\hline 6 & 2083.088 & 47990.37 & .19 & $\left({ }^{2} P\right) 5 d{ }^{3} D_{3}-\left({ }^{2} P\right) 4 f^{3} D_{3}$ & $\mathbf{I}$ & 1910.626 & 52338.88 & .87 & $(S) 65$ \\
\hline 5 & 2079.995 & 48061.73 & .58 & $\left({ }^{2} P\right) S d{ }^{3} P_{1}-\left({ }^{2} D\right) 4 f^{3} D_{1}$ & $\mathbf{0}$ & 1907.038 & 52437.33 & .47 & $\left({ }^{4} S\right) 6 S^{3} S_{1}-\left({ }^{2} P\right) 6 p^{3}$ \\
\hline 0 & 2077.610 & 48116.88 & 7.06 & $\left({ }^{4} S\right) 6 S^{3} S_{1}-\left({ }^{4} S\right) 4 f^{3} F_{2}$ & 6 & 1904.599 & 52504.50 & .54 & $\left({ }^{4} S\right) 5 d^{3} D_{3}-\left({ }^{4} S\right) 4 f^{3}$ \\
\hline 7 & 2075.090 & 48175.32 & .39 & $\left({ }^{2} D\right) 6 s^{3} D_{1}-\left({ }^{2} P\right) 6 p{ }^{3} P_{2}$ & $\mathbf{0}$ & 1901.779 & 52582.35 & .26 & $\left({ }^{4} S\right) 5 d^{S} D_{2}-\left({ }^{2} D\right) 6 p$ \\
\hline 7 & 2072.866 & 48226.99 & .97 & $\left({ }^{2} P\right) S d{ }^{1} D_{2}-\left({ }^{2} P\right) 6 p{ }^{3} D_{3}$ & 4 & 1900.693 & 52612.39 & .38 & $\left({ }^{2} P\right) S d d^{3} F_{3}-\left({ }^{2} P\right) 4 f^{3} G_{3}$ \\
\hline 0 & 2072.247 & 48241.41 & .40 & $\left({ }^{4} S\right) S d d^{5} D_{1}-\left({ }^{2} D\right) 6 p{ }^{3} F_{2}$ & 9 & 1899.216 & 52653.31 & 4.02 & $\left({ }^{2} D\right) 5 d^{3} D_{1}-\left({ }^{2} P\right) 6 p$ \\
\hline 7 & 2067.384 & 48354.87 & .73 & $\left({ }^{2} P\right) 5 d^{3} P_{0}-\left({ }^{2} D\right) 4 f^{3} D_{1}$ & 9 & 1899.216 & 52653.31 & .16 & $\left({ }^{2} P\right) 5 d d^{3} F_{2}-\left({ }^{2} P\right) 4 f^{3}$ \\
\hline 9 & 2067.175 & 48359.74 & .68 & $\left({ }^{2} D\right) S d{ }^{3} D_{1}-\left({ }^{2} D\right) 4 f^{3} F_{2}$ & 7 & 1899.156 & 52654.98 & 5.27 & $(S) 5 d{ }^{3} D_{2}-\left({ }^{2} D\right) 6 p p^{1} P_{1}$ \\
\hline 3 & 2060.702 & 48511.63 & .72 & $\left({ }^{2} P\right) 5 d^{3} D_{3}-\left({ }^{2} P\right) 4 f^{1} G_{4}$ & 10 & 1896.921 & 52717.02 & 6.95 & $\left({ }^{4} S\right) 5 d d^{3} D_{3}-\left({ }^{4} S\right) 4 \int^{3} F_{4}$ \\
\hline 0 & 2060.385 & 48519.10 & .01 & $5 s 5 p^{5} \quad{ }^{3} P_{2}-\left({ }^{4} S\right) 6 p{ }^{5} P_{1}$ & 11 & 1895.099 & 52767.68 & .40 & $\left({ }^{2} D\right) 5 d d^{3} F_{3}-\left({ }^{2} D\right) 4 f^{3} G_{4}$ \\
\hline 6 & 2055.034 & 48645.42 & .02 & $\left.{ }^{2} D\right) 5 d^{\prime} G_{4}-\left({ }^{2} D\right) 4 f^{3} H_{3}$ & $\mathbf{0}$ & 1892.723 & 52833.94 & .23 & $\left({ }^{4} S\right) S d{ }^{3} D_{3}-\left({ }^{2} D\right) 6 p{ }^{1} F_{3}$ \\
\hline 8 & 2052.729 & 48700.03 & 699.95 & $5 s 5 p^{5} \quad{ }^{3} P_{2}-\left({ }^{4} S\right) 6 p{ }^{5} P_{2}$ & 7 & 1888.374 & $\$ 2955.62$ & .67 & $\left({ }^{2} P\right) 5 d d^{3} P_{2}-\left({ }^{2} P\right) 4 f{ }^{3} D_{2}$ \\
\hline 5 & 2052.166 & 48713.39 & .44 & $\left({ }^{2} D\right) S d^{3} F_{4}-\left({ }^{2} D\right) 4 f^{3} G_{4}$ & 8 & 1888.083 & 52963.76 & .80 & $\left({ }^{2} D\right) 5 d d^{\prime} P_{1}-\left({ }^{2} D\right) 6 p{ }^{\prime} D_{2}$ \\
\hline 7 & 2051.875 & 48720.31 & .54 & $\left({ }^{2} D\right) 5 d^{3} P_{1}-\left({ }^{2} P\right) 4 f^{3} D_{2}$ & 11 & 1886.426 & 53010.29 & .07 & $\left({ }^{4} S\right) 5 d d^{3} D_{2}-\left({ }^{4} S\right) 4 f{ }^{3} F_{3}$ \\
\hline 8 & 2046.555 & 48846.93 & .94 & $\left({ }^{2} D\right) 6 s{ }^{3} D_{1}-\left({ }^{2} D\right) 4 f^{3} D_{2}$ & 3 & 1883.687 & 53087.38 & 6.87 & $\left({ }^{2} D\right) 5 d d^{3} D_{1}-\left({ }^{2} P\right) 6 p{ }^{3} P_{2}$ \\
\hline 1 & 2044.430 & 48897.70 & .73 & $\left({ }^{2} D\right) S d^{\prime} F_{3}-\left({ }^{4} S\right) S f^{5} F_{4}$ & 1 & 1882.957 & 53107.97 & 8.01 & $\left({ }^{2} D\right) 6 s{ }^{1} D_{2}-\left({ }^{2} P\right) 4 f^{3} F_{3}$ \\
\hline
\end{tabular}

Table I. Continued

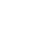


Table I. Continued

\begin{tabular}{|c|c|c|c|c|c|c|c|c|c|}
\hline \multirow[b]{2}{*}{ Intensity } & \multirow[b]{2}{*}{$\lambda(\boldsymbol{A})$} & \multicolumn{2}{|l|}{$\sigma\left(\mathrm{cm}^{-1}\right)$} & \multirow[b]{2}{*}{ Classification } & \multirow[b]{2}{*}{ Intensity' } & \multirow[b]{2}{*}{$\lambda(A)$} & \multicolumn{2}{|l|}{$\sigma\left(\mathrm{cm}^{-1}\right)$} & \multirow[b]{2}{*}{ Classification } \\
\hline & & obs & calc & & & & obs & calc & \\
\hline 1 & 1878.049 & 53246.74 & .80 & $\left({ }^{2} D\right) 5 d d^{3} G_{3}-\left({ }^{2} D\right) 4 f^{3} F_{4}$ & 10 & 1757.396 & 56902.37 & .37 & $\left({ }^{2} D\right) 5 d d^{3} F_{2}-\left({ }^{2} D\right) 4 f^{3} F_{2}$ \\
\hline 8 & 1875.878 & 53308.36 & .40 & $(S) 5 d{ }^{3} D_{1}-\left({ }^{2} P\right) 6 p{ }^{3} D_{1}$ & 11 & 1752.626 & 57057.24 & .59 & $\left({ }^{2} D\right) 5 d d^{3} G_{3}-\left({ }^{2} D\right) 4 f{ }^{3} F_{4}$ \\
\hline 10 & 1875.772 & 53311.38 & .14 & $\left.\left.{ }^{2} D\right) S d^{1} G_{4}-C^{2} D\right) 4 f^{1} H_{3}$ & 4 & 1752.318 & 57067.27 & .17 & $(\rho D) 5 d d^{\prime} G_{4}-\left({ }^{2} D\right) 4 f^{\prime} F_{3}$ \\
\hline 10 & 1874.915 & 53335.76 & .79 & $\left({ }^{2} D\right) S d^{3} G_{3}-\left({ }^{2} D\right) 4 f^{3} H_{4}$ & 4 & 1752.022 & 57076.90 & .50 & $\left({ }^{4} S\right) S d{ }^{3} D_{3}-(D D) 4 f^{3} G_{3}$ \\
\hline 9 & 1872.254 & 53411.57 & .47 & $\left({ }^{2} P\right) S d d^{\prime} D_{2}-\left({ }^{2} D\right) 4 f^{\prime} F_{3}$ & 5 & 1750.609 & 57122.99 & 3.09 & $5 s 5 p^{5} \quad{ }^{3} P_{1}-\left({ }^{2} D\right) 6 p{ }^{3} F_{2}$ \\
\hline 9 & 1870.665 & 53456.94 & .59 & ('P) ${ }^{2} d d^{1} D_{2}-\left({ }^{2} D\right) 4 f^{\prime} D_{2}$ & 9 & 1735.371 & 57642.56 & .60 & $\left({ }^{2} D\right) 5 d d^{3} G_{4}-\left({ }^{2} D\right) d f^{3} F_{6}$ \\
\hline 0 & 1869.447 & 53491.76 & .79 & ( $S) 5 d d^{3} D_{1}-\left({ }^{2} D\right) 6 p{ }^{3} P_{0}$ & 7 & 1734.368 & 57657.90 & .59 & $\left({ }^{4} S\right) 5 d{ }^{3} D_{3}-\left({ }^{2} D\right) 4 f{ }^{3} G_{4}$ \\
\hline 10 & 1866.547 & 53574.85 & .66 & $\left({ }^{2} D\right) S d^{3} G_{4}-\left({ }^{2} D\right) 4 f^{3} H_{3}$ & 3 & 1733.536 & 57685.55 & .68 & $\left({ }^{2} D\right) 5 d{ }^{3} F_{2}-\left({ }^{2} D\right) 4 f^{3} D_{3}$ \\
\hline 10 & 1865.157 & 53614.80 & .76 & $\left({ }^{2} D\right) 5 d^{3} F_{2}-\left({ }^{2} D\right) A f^{3} G_{3}$ & 5 & 1728.420 & 57856.31 & 5.89 & $\left({ }^{4} S\right) 6 s^{3} S_{1}-\left({ }^{2} D\right) 4 f^{\prime} P_{1}$ \\
\hline 4 & 1860.631 & 53745.21 & .14 & ( $D) 6 S^{3} D_{2}-\left({ }^{2} D\right) 4 \int^{3} P_{2}$ & 9 & 1727.429 & 57889.49 & .33 & $\left({ }^{2} D\right) 5 d{ }^{3} F_{3}-\left({ }^{2} P\right) 6 p$ \\
\hline 8 & 1860.181 & 53758.21 & .42 & $\left({ }^{2} D\right) S d d^{3} D_{1}-\left({ }^{2} D\right) 4 f^{3} D_{2}$ & 7 & 1724.983 & 57971.60 & .73 & $\left({ }^{2} D\right) 5 d^{3} G_{3}-\left({ }^{2} P\right) 6 p$ \\
\hline 9 & 1859.877 & 53766.99 & 7.01 & $\left({ }^{2} D\right) 6 s^{3} D_{3}-\left({ }^{2} D\right) 4 f^{\prime} G_{4}$ & 2 & 1724.776 & 57978.54 & .38 & $\left({ }^{4} S\right) 5 d{ }^{3} D_{4}-\left({ }^{\prime} S\right) 4 f$ \\
\hline 2 & 1856.569 & 53862.81 & .98 & $\left.{ }^{(} D\right) S d^{3} G_{s}-\left({ }^{2} D\right) 4 f^{1} H_{s}$ & 8 & 1722.358 & 58059.92 & .78 & $\left({ }^{2} P\right) 5 d{ }^{3} F_{2}-\left({ }^{2} P\right) 4 f$ \\
\hline 9 & 1855.196 & 53902.66 & .81 & $\left({ }^{2} D\right) S d^{3}{ }^{3} G_{4}-\left({ }^{2} D\right) 4 f^{3} H_{4}$ & 12 & 1717.515 & 58223.66 & 4.48 & $\left({ }^{2} P\right) 5 d{ }^{3} F_{4}-\left({ }^{2} P\right) 4 f$ \\
\hline 11 & 1854.375 & 53926.53 & .58 & ( $\left.{ }^{2} D\right) S d{ }^{3} G_{5}-\left({ }^{2} D\right) 4 f^{3} H_{6}$ & 5 & 1717.005 & 58240.95 & .78 & $\left({ }^{2} D\right) 5 d^{3} G_{4}-\left({ }^{2} D\right) 4 f^{\prime}$ \\
\hline 10 & 1853.884 & 53940.82 & .79 & $\left.{ }^{2} D\right) 5 d^{3} F_{4}-\left({ }^{2} D\right) 4 f^{3} G_{3}$ & 5 & 1716.290 & 58265.21 & .11 & $\left({ }^{2} D\right) 5 d{ }^{3} G_{3}-\left({ }^{2} D\right) 4 f$ \\
\hline 9 & 1852.583 & 53978.68 & .63 & ('P) $5 d d^{3} P_{2}-(C P) 4 \int^{3} D_{3}$ & 3 & 1713.867 & 58347.59 & .16 & $\left.{ }^{2} D\right) 5 d d^{\prime} F_{3}$ \\
\hline 6 & 1850.903 & 54027.68 & .85 & ('D) $5 d^{3} G_{3}-\left({ }^{2} D\right) 4 C^{3} D_{3}$ & 10 & 1712.522 & 58393.42 & .77 & $(S) S d{ }^{5} D_{2}-\left({ }^{4} S\right) 4 f$ \\
\hline 8 & 1848.989 & 54083.60 & .49 & $\left({ }^{4} S\right) S d d^{3} D_{4}-\left({ }^{4} S\right) 4 f^{5} F_{4}$ & 12 & 1705.178 & 58644.89 & .74 & $\left({ }^{4} S\right) S d d^{5} D_{3}-\left({ }^{4} s\right) \mathcal{A f}$ \\
\hline 9 & 1848.695 & 54092.20 & 1.94 & $\left({ }^{2} D\right) 5 d^{3} D_{2}-\left({ }^{2} P\right) 4 f^{3} F_{3}$ & 1 & 1704.946 & 58652.88 & .37 & $\left({ }^{2} D\right) 6 s^{3} 1$ \\
\hline 5 & 1837.300 & 54427.68 & .34 & $\left.{ }^{4} S\right) S d{ }^{5} D_{4}-\left({ }^{2} D\right) 6 p^{3} D_{3}$ & 8 & 1703.820 & 58691.66 & .76 & $555 p^{5} \quad 3$ \\
\hline 4 & 1837.223 & 54429.98 & 30.19 & $\left({ }^{4} S\right) S d{ }^{5} D_{1}-\left({ }^{\prime} S\right) 4 f^{s} F_{2}$ & 9 & 1699.754 & 58832.03 & .12 & $\left({ }^{2} D\right) 5 d^{3}$ \\
\hline 15 & 1835.811 & 54471.84 & 2.03 & $\left({ }^{4} S\right) 5 d{ }^{5} D_{4}-\left({ }^{4} S\right) 4 f$ & 10 & 1699.515 & 58840.31 & 39.78 & $5 s 5 p^{5} \quad 3$ \\
\hline 9 & 1835.532 & 54480.13 & 79.59 & $\left.{ }^{(} S\right) S d^{5} D_{0}-\left({ }^{4} S\right) 4 f^{5} F_{1}$ & 4 & 1696.936 & 58929.74 & .91 & $\left({ }^{2} D\right) S d$ \\
\hline 9 & 1834.255 & 54518.04 & 7.68 & $\left({ }^{4} S\right) S d{ }^{5} D_{2}-\left({ }^{4} S\right) 4 f$ & 7 & 1694.820 & 59003.30 & .31 & $\left({ }^{2} D\right) 5 d^{1}$ \\
\hline 1 & 1833.588 & 54537.87 & .62 & $\left.{ }^{2} D\right) 6 s{ }^{1} D_{2}-\left({ }^{4} S\right) 5 f$ & 7 & 1694.134 & 59027.21 & 8.51 & $\left({ }^{2} D\right) S d$ \\
\hline 1 & 1831.680 & 54594.69 & .87 & $\left({ }^{2} D\right) S d d^{3} G_{4}-\left({ }^{2} D\right) 4 f^{3} D_{3}$ & 9 & 1693.703 & 59042.24 & 1.96 & $\left({ }^{2} P\right) S d{ }^{3} F_{3}$ \\
\hline 9 & 1830.939 & 54616.78 & .43 & $\left({ }^{4} S\right) 5 d{ }^{5} D_{1}-\left({ }^{2} D\right) 6 p{ }^{3} P_{2}$ & 5 & 1691.798 & 59108.71 & .76 & $\left({ }^{2} D\right) 6 s^{3} D_{1}-\left({ }^{2} P\right) 4 f$ \\
\hline 9 & 1827.883 & 54708.10 & .09 & $\left({ }^{2} D\right) S d^{1} P_{1}-\left({ }^{4} S\right) 4 f^{3} F_{2}$ & 9 & 1685.823 & 59318.20 & 7.77 & $\left({ }^{2} D\right) 5 d{ }^{3} F_{2}-\left({ }^{2} P\right) 6 p$ \\
\hline 9 & 1827.367 & 54723.55 & .64 & $\left({ }^{4} S\right) S d{ }^{5} D_{1}-\left({ }^{4} S\right) 4 f^{5} F_{1}$ & 2 & 1684.194 & 59375.59 & .06 & $\left({ }^{2} P\right) 6 s{ }^{3} F$ \\
\hline 12 & 1826.490 & 54749.82 & .86 & $\left({ }^{4} S\right) S d d^{3} D_{3}-\left({ }^{4} S\right) 4 f^{3} F_{4}$ & 6 & 1678.873 & 59563.78 & .49 & (2P) $5 d{ }^{3} /$ \\
\hline 12 & 1826.490 & 54749.82 & .74 & $\left.{ }^{4} S\right) S d{ }^{3} D_{2}-\left({ }^{2} D\right) 6 p{ }^{1} D_{2}$ & 11 & 1675.824 & 59672.13 & 0.95 & $\left({ }^{4} S\right) 5 d^{3} D_{1}-\left({ }^{2} D\right) 4 f^{3}$ \\
\hline 9 & 1825.867 & 54768.49 & .65 & $\left({ }^{4} S\right) 5 d d^{3} D_{3}-\left({ }^{4} S\right) 4 \int^{s} F_{3}$ & 11 & 1674.633 & 59714.57 & .89 & $\left({ }^{2} D\right) 6 s{ }^{3} D_{1}-\left({ }^{4} S\right) 5 f$ \\
\hline 11 & 1823.391 & 54842.88 & .73 & $\left.\left({ }^{4} S\right) 5 d{ }^{5} D_{2}-{ }^{2} D\right) 6 p{ }^{3} D_{3}$ & 9 & 1673.566 & 59752.66 & .24 & $5 s 5 p^{5} \quad 3$ \\
\hline 10 & 1821.334 & 54904.82 & 5.09 & $\left({ }^{2} D\right) 6 s \quad D_{2}-\left({ }^{2} P\right) 4 f^{3} G_{3}$ & 9 & 1672.557 & 59788.71 & .73 & $\left.{ }^{2} P\right) 5 d !$ \\
\hline 11 & 1819.857 & 54949.37 & .41 & $(S) S d d^{3} D_{3}-\left({ }^{2} D\right) 6 p^{3} F_{4}$ & 7 & 1669.355 & 59903.37 & · .12 & $\left({ }^{2} D\right) S d{ }^{3} F_{2}$ \\
\hline 9 & 1817.397 & 55023.74 & .71 & $\left.{ }^{4} S\right) 5 d{ }^{3} D_{2}-\left({ }^{4} S\right) 4 f^{3} F_{2}$ & 9 & 1663.390 & 60118.18 & 7.76 & ('S)6s st \\
\hline 11 & 1815.085 & 55093.85 & .70 & $\left({ }^{4} S\right) 5 d{ }^{5} D_{3}-\left({ }^{2} D\right) 6 p{ }^{3} D_{3}$ & 6 & 1661.093 & 60201.31 & .11 & $\left({ }^{2} D\right) 5 d d^{3} F_{3}-\left({ }^{2} P\right) 6 p$ \\
\hline 10 & 1814.503 & 55111.51 & .53 & $\left({ }^{2} D\right) 6 s^{3} D_{2}-\left({ }^{2} D\right) 4 f^{\prime} F_{3}$ & 1 & 1661.029 & 60203.63 & .67 & $\left.\left({ }^{2} D\right) 5 d^{3} D_{3}-\left({ }^{2} P\right) 4\right)^{3}$ \\
\hline 3 & 1812.301 & SS 178.48 & .55 & $\left({ }^{2} D\right) S d d^{3} D_{1}-\left({ }^{2} D\right) 4 f^{3}{ }^{3} P_{2}$ & 4 & 1660.808 & 60211.67 & .39 & $\left({ }^{4} S\right) 5 d d^{3} D_{1}-\left({ }^{2} P\right) 6 p$ \\
\hline 11 & 1811.263 & 55210.08 & 09.94 & $\left({ }^{4} S\right) 5 d{ }^{3} D_{2}-\left({ }^{2} D\right) 6 p{ }^{3} P_{2}$ & 10 & 1656.612 & 60364.15 & .12 & $\left({ }^{4} S\right) 5 d{ }^{3} D_{3}-\left({ }^{2} D\right) 4 f$ \\
\hline 11 & 1810.520 & 55232.75 & 3.01 & $\left({ }^{2} D\right) 5 d{ }^{3} F_{4}-\left({ }^{2} D\right) 4 f^{3} F_{4}$ & 3 & 1656.069 & 60383.96 & 4.41 & $(S) S d d^{3} D_{3}-\left({ }^{2} D\right) 6 p$ \\
\hline 7 & 1807.746 & 55317.51 & .16 & $\left({ }^{4} S\right) 5 d{ }^{5} D_{2}-\left({ }^{4} S\right) 4 f$ & 10 & 1653.039 & 60494.65 & .49 & $\left({ }^{2} D\right) 5 d{ }^{3} F_{3}$ \\
\hline 4 & 1805.316 & 55391.96 & 2.05 & $\left({ }^{4} S\right) 5 d^{5} D_{0}-\left({ }^{2} D\right) 6 p{ }^{3} P_{1}$ & 10 & 1647.338 & 60704.01 & 3.82 & $\left({ }^{4} S\right) 6 s{ }^{3} S_{1}$ \\
\hline 10 & 1804.117 & 55428.78 & .78 & $555 p^{5}{ }^{3} P_{1}-\left(^{2} D\right) 6 p{ }^{3} D_{1}$ & 8 & 1647.010 & 60716.09 & 5.85 & $\left.{ }^{4} S\right) 5 d d^{3} D$ \\
\hline 8 & 1803.073 & 55460.87 & .91 & $\left({ }^{4} S\right) 5 d d^{5} D_{3}-\left({ }^{2} D\right) 6 p^{3} P_{2}$ & 2 & 1646.504 & 60734.75 & .51 & $\left({ }^{2} D\right) 6 p^{3}$ \\
\hline 9 & 1799.900 & 55558.64 & .50 & $\left.{ }^{2} D\right) S d^{3} D_{1}-\left({ }^{2} D\right) 4 f^{3} D_{1}$ & 10 & 1642.767 & 60872.91 & .66 & $\left({ }^{2} D\right) 5 d{ }^{3} F_{3}$ \\
\hline 7 & 1799.769 & 55562.68 & .50 & $\left({ }^{2} D\right) S d{ }^{3} S_{1}-\left({ }^{2} P\right) 4 f^{3} D_{2}$ & 3 & 1642.395 & 60886.68 & .77 & $\left.{ }^{2} P\right) 5 d^{\prime} D_{2}-\left({ }^{2} P\right) 4 f$ \\
\hline 10 & 1799.691 & 55565.08 & .17 & $\left({ }^{2} D\right) 5 d^{3} F_{3}-\left({ }^{2} D\right) 4 f^{3} H_{4}$ & 3 & 1637.572 & 61066.01 & .00 & $\left({ }^{(} S\right) S d d^{3} D_{2}-\left({ }^{2} D\right) 4 f^{3}$ \\
\hline 6 & 1790.544 & 55848.96 & 9.19 & $\left({ }^{2} D\right) S d^{3} F_{4}-\left({ }^{2} D\right) 4 f^{1} H_{3}$ & 11 & 1635.382 & 61147.81 & .43 & $\left.{ }^{4} S\right) 5 d^{3} D_{3}$ \\
\hline 7 & 1789.258 & 55889.09 & .02 & $\left({ }^{2} D\right) 5 d^{3} D_{2}-\left({ }^{2} P\right) 4 f^{3} G_{3}$ & 3 & 1634.072 & 61196.81 & .71 & $\left.{ }^{(} D\right) S d{ }^{3} F_{2}$ \\
\hline 8 & 1786.678 & 55969.79 & 70.03 & $\left({ }^{2} D\right) S d d^{\prime} F_{3}-\left({ }^{2} P\right) 4 f^{3} D_{3}$ & 7 & 1633.489 & 61218.65 & .34 & $\left.{ }^{2} P\right) S d^{\prime} D_{2}-\left({ }^{4} S\right) S f^{5}$ \\
\hline 2 & 1785.311 & 56012.64 & .74 & $\left.{ }^{2} D\right) 5 d{ }^{3} P_{2}-\left({ }^{2} P\right) 4 f^{3} D_{3}$ & 10 & 1633.255 & 61227.42 & 6.63 & $\left({ }^{2} D\right) 5 d^{3} D_{3}-\left({ }^{2} P\right) 4 f^{3}$ \\
\hline 8 & 1784.661 & 56033.05 & .18 & $\left({ }^{4} S\right) S d^{3} D_{1}-\left({ }^{2} P\right) 6 p{ }^{3} D_{2}$ & 10 & 1631.429 & 61295.96 & .64 & $\left({ }^{2} D\right) S d d^{3} D_{2}-\left({ }^{2} P\right) 4 f^{3} D_{2}$ \\
\hline 3 & 1780.067 & 56177.66 & .89 & $5 s 5 p^{5} \quad{ }^{3} P_{0}-\left(^{2} D\right) 6 p p^{1} P_{1}$ & 6 & 1629.312 & 61375.60 & .37 & $\left({ }^{4} S\right) 6 s^{3} S_{1}-\left({ }^{2} D\right) 4 f^{3}$ \\
\hline 9 & 1778.783 & 56218.22 & .12 & $\left(^{2} D\right) 5 d^{3} P_{1}-5 s^{0} 5 p^{6} \quad S_{0}$ & 1 & 1628.148 & 61419.48 & .09 & $\left.{ }^{2} P\right) 5 d^{3}$ \\
\hline 2 & 1788.425 & 56229.54 & .62 & $\left({ }^{4} S\right) 5 d d^{5} D_{2}-\left({ }^{2} D\right) 6 p{ }^{3} P_{1}$ & 1 & 1626.309 & 61488.93 & .79 & $\left.{ }^{2} D\right) 6 s^{3}$ \\
\hline 11 & 1777.910 & 56245.82 & .29 & $\left({ }^{2} D\right) S d{ }^{3} G_{3}-\left({ }^{2} P\right) 6 p{ }^{3} D_{3}$ & 3 & 1626.183 & 61493.68 & 2.90 & $\left({ }^{2} P\right) S d d^{1} D_{2}-\left({ }^{4} S\right) S f$ \\
\hline 6 & 1777.551 & 56257.19 & .24 & $\left({ }^{2} D\right) 5 d d^{3} F_{3}-\left({ }^{2} D\right) 4 f^{3} D_{3}$ & 5 & 1624.678 & 61550.66 & .20 & $\left({ }^{4} S\right) S d d^{3} D_{1}-\left({ }^{2} D\right) 4 f^{\prime}$ \\
\hline 9 & 1775.174 & 56332.50 & .39 & $\left({ }^{2} D\right) 5 d^{3} G_{4}-\left({ }^{2} D\right) 4 f^{3} G_{9}$ & 6 & 1623.748 & 61585.90 & .81 & $\left({ }^{2} P\right) 5 d^{1} D_{2}-\left({ }^{2} P\right) 4 f^{3} G_{3}$ \\
\hline $\boldsymbol{9}$ & 1774.033 & 56368.75 & .88 & $\left({ }^{2} P\right) S d d^{3} F_{4}-\left({ }^{2} P\right) 4 f^{\prime} G_{4}$ & 8 & 1622.595 & 61629.67 & .56 & $\left({ }^{2} D\right) 5 d d^{3} F_{2}-\left({ }^{2} P\right) 6 p{ }^{3} P_{2}$ \\
\hline 5 & 1771.778 & 56440.47 & .53 & $\left({ }^{2} D\right) S d d^{3} F_{4}-\left({ }^{2} D\right) 4 f^{3} F_{3}$ & 7 & 1621.407 & 61674.84 & .75 & $(S) S d d^{3} D_{4}-\left({ }^{\prime} S\right) 4 f^{3}$ \\
\hline 0 & 1770.919 & 56467.87 & .45 & $\left.{ }^{2} D\right) S d{ }^{3} D_{1}-\left({ }^{2} D\right) A f^{3} P_{1}$ & 8 & 1619.484 & 61748.05 & .16 & $\left.{ }^{2} D\right) 5 d^{3} D_{3}-\left({ }^{2} P\right) 4 f^{1} G_{4}$ \\
\hline 10 & 1770.174 & 56491.62 & .56 & $\left({ }^{2} D\right) S d d^{\prime} F_{3}-\left({ }^{2} P\right) 4 f^{1} G_{4}$ & 3 & 1616.083 & 61878.00 & 7.74 & $\left({ }^{4} S\right) S d{ }^{3} D_{2}-\left({ }^{4} S\right) 4 \int^{3} F_{2}$ \\
\hline 10 & 1770.099 & 56494.01 & .03 & $\left({ }^{4} S\right) S d{ }^{3} D_{2}-\left({ }^{\prime} S\right) 4 f^{3} F_{2}$ & 6 & 1612.970 & 61997.45 & 6.81 & $\left({ }^{2} D\right) 5 d^{3} G_{4}-\left({ }^{2} D\right) 4 f^{\prime}$ \\
\hline 10 & 1767.089 & 56590.26 & .05 & $\left({ }^{2} D\right) 5 d^{3} D_{1}-\left({ }^{2} D\right) 4 f^{\prime} D_{2}$ & 6 & 1612.970 & 61997.45 & .01 & $(S) 6 s^{5} S_{2}-\left({ }^{2} D\right) 4 f^{\prime}$ \\
\hline 6 & 1760.178 & 56812.45 & .31 & $\left({ }^{2} D\right) 5 d^{3} G_{4}-\left({ }^{2} P\right) 6 p{ }^{3} D_{3}$ & 5 & 1610.658 & 62086.42 & .35 & $(S) S d d^{3} D$ \\
\hline $\mathbf{0}$ & 1759.622 & 56830.40 & .14 & $\left({ }^{4} S\right) 6 s^{3} S_{2}-\left({ }^{2} D\right) 4 f^{3}{ }^{3}$, & 2 & 1606.393 & 62251.26 & .48 & $\left({ }^{2} D\right) 5 d^{3} F_{4}-\left({ }^{2} D\right) 4 f^{\prime} G_{4}$ \\
\hline
\end{tabular}

Table I. Continued

Table I. Continued


Table I. Continued

$\frac{\sigma\left(\mathrm{cm}^{-1}\right)}{\text { Intensity' }} \quad \lambda(A) \quad$ obs calc Classification

$\begin{array}{lllll}9 & 1605.322 & 62292.78 & .79 & \left({ }^{2} D\right) 5 d{ }^{3} F_{3}-\left({ }^{2} D\right) 4 f^{3} P_{2}\end{array}$

$9 \begin{array}{lllll}9 & 1605.322 & 62292.78 & .79 & \left({ }^{2} D\right) 5 d{ }^{3} F_{3}-\left({ }^{2} D\right) 4 f^{3} P_{2}\end{array}$

$\begin{array}{lllll}10 & 1605.106 & 62301.19 & .10 & \left({ }^{2} D\right) S d{ }^{3} F_{2}-\left({ }^{2} D\right) 4 f{ }^{3} D_{2}\end{array}$

$\begin{array}{lllll}8 & 1604.074 & 62341.26 & .12 & \left({ }^{4} S\right) S d{ }^{3} D_{3}-\left({ }^{4} S\right) 4 f{ }^{3} F_{4} \\ 10 & 1603.241 & 62373.67 & .48 & 5 s 5 p^{5}{ }^{3} P_{1}-\left({ }^{2} D\right) 6 p{ }^{3} P_{0}\end{array}$

$\begin{array}{lllrll}10 & 1603.241 & 62373.67 & .48 & 5 s 5 p^{5} & { }^{3} P_{1}-\left({ }^{2} D\right) 6 p{ }^{3} P_{0} \\ 9 & 1601.817 & 62429.09 & 8.83 & 5 s 5 p^{5} & { }^{3} P_{2}-\left({ }^{2} D\right) 6 p{ }^{3} F_{2}\end{array}$

$11 \quad \begin{array}{llll}598.260 & 62568.05 & 7.67 & \left({ }^{2} D\right) 5 d{ }^{1} P_{1}-\left({ }^{2} D\right) 4{ }^{3} F_{2}\end{array}$

$\begin{array}{llllrl}11 & w & 1596.687 & 62629.68 & 8.98 & \left({ }^{2} P\right) 5 d{ }^{3} P_{1}-\left({ }^{2} P\right) 4 f \\ 10 & & 1592.466 & 62795.69 & .50 & \left({ }^{4} S\right) 6 .{ }^{3} D_{1}-\left({ }^{2} D\right) 4 f^{3} P_{2}\end{array}$

$10 \quad 1592.466$

4

12

9

9

9

1569.753
$10 \quad 1569.338$

12

$9 \quad 1562.006$

$10 \quad 1560.437$

$11 \quad$ I560.01

$7 \quad 1558.178$

$5 \quad 1557.460$

$8 \quad 1554.426$

$10 \quad 1553.907$

$10 \quad 1551.651$

9

1549.961

I548.008

1547.346

1546.952

1540.970

1538.863

1538.220

1536.812

1536.386

1535.328

1535.224

1533.416

I532.765

1530.558

1526.586

1524.280

I521.501

1520.619

1515.558

1511.119

1509.841

1509.530

1509.458

I508.178

1504.900

1503.997

1501.157

1497.708

1495.446

1488.474

1486.291

1484.688

w $\quad 1482.243$

1474.781

1472.727

1471.312

1468.378

1468.178

1462.549

1461.206

1458.806
62795.69
62918.75

63060.83

63108.33

63312.15

63365.16

63498.22

63605.62

63659.19

63704.28

63721.14

63998.06

64020.25

64084.60

64102.10

64177.53

64207.12

64332.43

64353.91

64447.50

64517.77

64599.14

64626.80

64643.24

64894.18

64983.05

65010.22

65069.76

65087.81

65132.65

65137.09

65213.86

65241.58

65335.64

65505.65

65604.76

65724.58

65762.71

65982.31

66176.14

66232.15

66245.78

66248.96

66305.15

66449.59

66489.51

66615.30

66768.67

66869.67

67182.92

67281.56

67354.20

67465.34

67806.69

67901.25

67966.57

68102.35

68111.62

68373.79

68436.61

68549.23
$.40 \quad\left({ }^{2} D\right) 6 s^{3} D_{2}-\left({ }^{4} S\right) S f^{5} F_{3}$

$.07\left({ }^{2} D\right) 5 d d^{3} S_{1}-5 s^{0} 5 p^{6}{ }^{1} S_{0}$

$\left..12 \quad{ }^{2} D\right) 5 d d^{1} P_{1}-\left({ }^{2} P\right) 6 p{ }^{3} S_{1}$

$1.885 s 5 p^{5} \quad{ }^{3} P_{1}-\left({ }^{4} S\right) 4 f{ }^{3} F_{2}$

4.87 ( $S$ ) $S d{ }^{3} D_{3}-\left({ }^{2} P\right) 6 p{ }^{3} D_{3}$

$.125 s 5 p^{5} \quad{ }^{3} P_{1}-\left({ }^{2} D\right) 6 p{ }^{3} P_{2}$

$.335 s 5 p^{5} \quad{ }^{3} P_{1}-\left({ }^{4} S\right) 4 f^{5} F_{1}$

$.17 \quad\left({ }^{2} D\right) 5 d^{3} F_{3}-\left({ }^{2} D\right) 4 f{ }^{1} F_{3}$

$.29 \quad\left({ }^{2} D\right) 5 d^{3} F_{3}-\left({ }^{2} D\right) 4 f^{1} D_{2}$

$.24 \quad\left({ }^{2} D\right) 5 d{ }^{3} F_{2}-\left({ }^{2} D\right) 4{ }^{3} P_{2}$

$7.50 \quad 5 s 5 p^{3} \quad{ }^{3} P_{2}-\left({ }^{2} D\right) 6 p^{3} D_{2}$

$.24 \quad\left({ }^{2} D\right) 5 d^{3} D_{1}-\left({ }^{2} P\right) 4 f^{3} F_{2}$

$.40 \quad\left({ }^{4} S\right) 6 s^{3} S_{1}-\left({ }^{2} D\right) 4 f{ }^{3} P_{1}$

1.19 ( $\left.{ }^{2} D\right) S d{ }^{3} F_{2}-\left({ }^{2} D\right) 4 f{ }^{3} D_{1}$

$.16 \quad\left({ }^{4} S\right) S d{ }^{3} D_{3}-\left({ }^{2} D\right) 4 f{ }^{3} F_{4}$

$.00 \quad\left({ }^{4} S\right) 6 s^{3} S_{1}-\left({ }^{2} D\right) 4 f^{1} D_{2}$

$.345 s 5 p^{5} \quad{ }^{3} P_{2}-\left({ }^{2} D\right) 6 p{ }^{3} F_{3}$

$.62\left({ }^{4} S\right) 5 d{ }^{3} D_{2}-\left({ }^{2} D\right) 4 f^{3} F_{2}$

6.92 ( $\left.{ }^{2} D\right) 5 d{ }^{1} P_{1}-\left({ }^{2} D\right) 4 f^{1} P_{1}$

$.795 s 5 p^{5}{ }^{3} P_{1}-\left({ }^{2} D\right) 6 p{ }^{3} P_{1}$

$8.79\left({ }^{2} D\right) S d^{1} G_{4}-\left({ }^{4} S\right) 5 f^{5} F_{4}$

$.37 \quad\left({ }^{2} D\right) S d^{3} D_{1}-\left({ }^{4} S\right) S f{ }^{5} F_{2}$

$.07\left({ }^{2} D\right) 5 d^{3} G_{4}-\left({ }^{2} D\right) 4 f^{1} G_{4}$

$.06\left({ }^{4} S\right) S d{ }^{3} D_{2}-\left({ }^{2} P\right) 6 p{ }^{3} S_{1}$

$.07 \quad\left({ }^{2} D\right) 5 d^{\prime} P_{1}-\left({ }^{2} P\right) 6 p{ }^{1} D_{2}$

$.13 \quad\left({ }^{2} D\right) S d{ }^{3} F_{2}-\left({ }^{2} D\right) 4 f^{3} P_{1}$

$.68 \quad(S) 5 d^{3} D_{1}-\left({ }^{2} D\right) 4 f{ }^{3} D_{2}$

.62 ( $\left.{ }^{2} D\right) S d^{3} F_{2}-\left({ }^{2} D\right) 4 f^{1} F_{3}$

$.74 \quad\left({ }^{2} D\right) 5 d^{3} F_{2}-\left({ }^{2} D\right) 4 f^{1} D_{2}$

$6.93\left({ }^{4} S\right) S d{ }^{3} D_{2}-\left({ }^{2} D\right) 4 f^{3} D_{3}$

4.42 ( $\left.{ }^{2} D\right) 6 s^{3} D_{1}-\left({ }^{2} P\right) 4{ }^{3} D_{2}$

$.51 \quad\left({ }^{2} D\right) 5 d^{1} G_{4}-\left({ }^{2} P\right) 4 f^{3} G_{3}$

$.39 \quad\left({ }^{4} S\right) 5 d d^{5} D_{0}-\left({ }^{2} P\right) 6 p{ }^{3} P_{1}$

$6.04\left({ }^{4} S\right) S d{ }^{5} D_{1}-\left({ }^{2} P\right) 6 p{ }^{3} D_{2}$

$.64 \quad\left({ }^{4} S\right) 5 d{ }^{5} D_{1}-\left({ }^{2} P\right) 6 p{ }^{3} P_{0}$

.67 (2D)6s ${ }^{3} D_{3}-\left({ }^{2} P\right) 4 f^{3} D_{3}$

.85 ( $S) 5 d d^{3} D_{3}-\left({ }^{2} D\right) 4 f^{3} D_{2}$

$.48\left({ }^{2} D\right) 5 d{ }^{3} F_{4}-\left({ }^{2} P\right) 4 f{ }^{3} F_{3}$

$.175 s 5 p^{5}{ }^{3} P_{2}-\left({ }^{2} D\right) 6 p{ }^{1} F_{3}$

$.87(S) 5 d^{3} D_{2}-\left({ }^{2} D\right) 4 f^{\prime} P_{1}$

6.20 ( $\left.{ }^{2} D\right) 6{ }^{3} D_{3}-\left({ }^{2} P\right) 4 f{ }^{1} G_{4}$

$9.185 s 5 p^{5}{ }^{3} P_{2}-\left({ }^{2} D\right) 6 p{ }^{1} P_{1}$

$.44 \quad\left({ }^{2} D\right) 5 d^{3} F_{3}-\left({ }^{2} D\right) 4 f^{1} G_{4}$

$.70\left({ }^{4} S\right) S d{ }^{5} D_{2}-\left({ }^{2} D\right) 4 f^{3} G_{3}$

$.81\left({ }^{4} S\right) S d{ }^{3} D_{1}-\left({ }^{2} D\right) 4 f^{3} P_{2}$

$.40\left({ }^{4} S\right) 5 d^{5} D_{4}-\left({ }^{2} D\right) 4 f^{3} G_{4}$

$9.02\left({ }^{4} S\right) S d{ }^{3} D_{2}-\left({ }^{2} P\right) 6 p{ }^{1} D_{2}$

$.76(S) S d d^{3} D_{1}-\left({ }^{2} D\right) 4 f^{3} D_{1}$

$.98\left({ }^{4} S\right) 5 d^{3} D_{3}-\left({ }^{2} D\right) 4 \int^{3} P_{2}$

$.76\left({ }^{4} S\right) S d d^{3} D_{3}-\left({ }^{2} D\right) 4 j{ }^{3} G_{4}$

$.36\left({ }^{4} S\right) 5 d{ }^{3} D_{2}-\left({ }^{2} P\right) 6 p{ }^{3} D_{3}$

$.265 s^{2} 5 p^{4}{ }^{1} S_{0}-5 s 5 p^{5} \quad{ }^{3} P_{1}$

$7.06 \quad\left({ }^{2} D\right) 5 d{ }^{3} G_{3}-\left({ }^{2} P\right) 4 f{ }^{3} F_{3}$

$.31(S) S d{ }^{3} D_{1}-\left({ }^{2} D\right) 4 f^{1} D_{2}$

$\left..40 \quad{ }^{2} D\right) 5 d^{1} P_{1}-\left({ }^{2} D\right) 4 f^{3} D_{2}$

1.80 ( $\left.{ }^{2} D\right) 6 s{ }^{3} D_{3}-\left({ }^{2} P\right) 4 f{ }^{3} F_{4}$

$.595 s 5 p^{5}{ }^{3} P_{2}-\left({ }^{4} S\right) 4 f^{5} F_{3}$

4.07 ( $2 D) 5 d{ }^{3} G_{4}-\left({ }^{2} P\right) 4 f{ }^{3} F_{3}$

$.645 s 5 p^{3}{ }^{3} P_{2}-\left({ }^{2} D\right) 6 p{ }^{3} D_{3}$

$.36\left({ }^{4} S\right) S d{ }^{3} D_{3}-\left({ }^{2} D\right) 4 f^{1} F_{3}$

Table I. Continued

\begin{tabular}{|c|c|c|c|c|c|}
\hline \multirow{2}{*}{\multicolumn{2}{|c|}{ Intensity }} & \multirow[b]{2}{*}{$\lambda(A)$} & \multicolumn{2}{|l|}{$\sigma\left(\mathrm{cm}^{-1}\right)$} & \multirow[b]{2}{*}{ Classification } \\
\hline & & & obs & calc & \\
\hline 6 & & 1457.845 & 68594.40 & .48 & $\left({ }^{4} S\right) 5 d{ }^{3} D$ \\
\hline 8 & & 1457.351 & 68617.66 & .62 & $-\left({ }^{4} S\right) 4 f^{5} F_{2}$ \\
\hline 1 & & 1455.769 & 68692.20 & .49 & $-\left({ }^{2} P\right) 4{ }^{3} D_{2}$ \\
\hline 7 & & 1453.408 & 68803.79 & .85 & $-\left({ }^{2} D\right) 6 p{ }^{3} P_{2}$ \\
\hline 3 & & 1451.141 & 68911.30 & .07 & $-\left({ }^{4} S\right) 4 f{ }^{5} F_{1}$ \\
\hline 7 & & 1450.093 & 68961.10 & .42 & $\left({ }^{2} D\right) S d{ }^{3} G_{3}-\left({ }^{4} S\right) S f^{5} F_{4}$ \\
\hline 8 & & 1447.580 & 69080.82 & .80 & $\left({ }^{4} S\right) S d{ }^{3} D_{2}-\left({ }^{2} P\right) 6 p{ }^{3} P_{2}$ \\
\hline 5 & & 1447.487 & 69085.26 & .02 & $-\left({ }^{2} D\right) 4 f{ }^{3} H_{5}$ \\
\hline 8 & & 1446.268 & 69143.49 & .80 & $\left({ }^{2} D\right) 4 f^{3} F_{2}$ \\
\hline 2 & & 1441.199 & 69386.67 & .53 & $P_{1}-\left({ }^{2} D\right) 4 f^{3} P_{2}$ \\
\hline 2 & & 1440.650 & 69413.11 & .17 & $\left({ }^{2} D\right) 4 f{ }^{3} H_{4}$ \\
\hline 7 & & 1440.089 & 69440.17 & .20 & $\left({ }^{4} S\right) S d{ }^{5} D_{0}-\left({ }^{2} P\right) 6 p{ }^{3} S_{1}$ \\
\hline 1 & & 1438.259 & 69528.49 & .43 & $\left({ }^{2} D\right) S d{ }^{3} G_{4}-\left({ }^{4} S\right) S f{ }^{5} F_{4}$ \\
\hline 6 & & 1436.707 & 69603.61 & 4.14 & $(D) 5 d{ }^{3} G_{3}$ \\
\hline 7 & & 1435.045 & 69684.23 & .25 & $\left.{ }^{2} P\right) 6 p{ }^{3} S_{1}$ \\
\hline 2 & & 1434.820 & 69695.13 & .57 & $-\left({ }^{2} P\right) 6 p{ }^{3} P_{1}$ \\
\hline 3 & & 1433.956 & 69737.16 & .32 & $-\left({ }^{2} D\right) 4 f^{3} F_{2}$ \\
\hline 9 & & 1432.185 & 69823.37 & .53 & $\left({ }^{2} D\right) 6 p{ }^{3} P_{1}$ \\
\hline 8 & & 1428.810 & 69988.31 & .29 & $\left({ }^{2} D\right) 4 f^{3} F_{2}$ \\
\hline 7 & & 1427.844 & 70035.68 & 6.44 & $\left({ }^{2} D\right) S d^{3}$ \\
\hline 10 & & 1425.999 & 70126.29 & .56 & $\left({ }^{2} P\right) S d{ }^{3}$ \\
\hline 6 & & 1425.200 & 70165.59 & .91 & $5 s 5 p^{3} \quad 3$ \\
\hline 8 & & 1418.027 & 70520.51 & .63 & $\left({ }^{2} D\right) 4 f^{3} D_{3}$ \\
\hline 10 & & 1414.921 & 70675.30 & .43 & $-\left({ }^{2} D\right) 4 f^{3} P_{1}$ \\
\hline 0.5 & & 1413.006 & 70771.09 & .60 & $-\left({ }^{2} D\right) 4 f^{3} D_{3}$ \\
\hline 0.5 & & 1412.846 & 70779.11 & .00 & $\left({ }^{4} S\right) S d{ }^{3} D_{0}-\left({ }^{2} D\right) 4 f^{1} P_{1}$ \\
\hline 15 & & 1412.466 & 70798.14 & .04 & $\left({ }^{2} D\right) 5 d^{1} P_{1}-\left({ }^{2} D\right) 4 f^{1} D_{2}$ \\
\hline 4 & & 1407.990 & 71023.22 & .05 & $\left.{ }^{4} S\right) 5 d^{5} D_{1}-\left({ }^{2} D\right) 4 f^{1} P_{1}$ \\
\hline 8 & & 1405.042 & 71172.27 & .48 & $\left({ }^{4} S\right) S d{ }^{3} D_{2}-\left({ }^{2} D\right) 4 f^{3} P_{2}$ \\
\hline 2 & & 1404.681 & 71190.55 & .80 & $\left({ }^{2} D\right) S d^{3} F_{3}-\left({ }^{4} S\right) 5 f{ }^{5} F_{4}$ \\
\hline 3 & & 1404.586 & 71195.37 & .63 & $\left({ }^{4} S\right) 5 d^{3} D_{3}-\left({ }^{2} D\right) 4 f^{1} G_{4}$ \\
\hline 8 & & 1402.224 & 71315.26 & .15 & $\left.{ }^{2} P\right) 6 p{ }^{1} D_{2}$ \\
\hline 4 & & 1399.271 & 71465.79 & 4.88 & $\left({ }^{2} D\right) S d d^{3} F$ \\
\hline 4 & & 1399.271 & 71465.79 & .13 & $\left.{ }^{2} D\right) 5 d^{2}$ \\
\hline 4 & & 1399.271 & 71465.79 & 6.05 & $\left({ }^{2} D\right) 5 d^{3}$ \\
\hline 2 & $\mathbf{u}$ & 1397.573 & 71552.63 & .43 & $\left.{ }^{2} D\right) 4 f^{3} D_{1}$ \\
\hline 1 & & 1392.122 & 71832.78 & 3.52 & $\left({ }^{2} D\right) 5 d^{3}$ \\
\hline 5 & & 1391.936 & 71842.37 & .75 & $\left.{ }^{2} D\right) 4 f^{3} G_{5}$ \\
\hline 11 & & 1389.129 & 71987.53 & .68 & $\left.{ }^{4} S\right) 4 f^{3} F_{3}$ \\
\hline 9 & & 1380.055 & 72460.90 & 1.38 & $\left.{ }^{2} D\right) 4{ }^{3} P_{1}$ \\
\hline 3 & & 1378.128 & 72562.23 & .92 & $\left({ }^{2} P\right) 4 f{ }^{3} F_{2}$ \\
\hline 10 & & 1377.722 & 72583.58 & .98 & $\left.{ }^{2} D\right) 4 f^{\prime} D_{2}$ \\
\hline 8 & & 1375.295 & 72711.67 & 2.00 & $-5 s^{0} s p^{6} \quad S_{0}$ \\
\hline 3 & & 1374.809 & 72737.36 & 8.07 & $\left({ }^{4} S\right) 5 d{ }^{3} D_{2}$ \\
\hline 0.5 & $\mathbf{h}$ & 1370.076 & 72988.64 & 9.04 & $(S) 5 d{ }^{5} D_{3}$ \\
\hline 8 & & 1366.709 & 73168.47 & 9.05 & $\left({ }^{2} D\right) 5 d{ }^{3} F_{2}$ \\
\hline 3 & & 1364.977 & 73261.32 & .96 & $\left({ }^{2} D\right) 5 d$ \\
\hline 11 & & 1356.358 & 73726.86 & 7.35 & $5 s 5 p^{3}$ \\
\hline 10 & & 1355.012 & 73800.10 & .38 & P) $6 p{ }^{3} S_{1}$ \\
\hline 6 & & 1347.545 & 74209.01 & .14 & $\left({ }^{2} D\right) S d$ \\
\hline 5 & & 1344.320 & 74387.03 & .73 & $5 s 5 p^{3}$ \\
\hline 6 & & 1342.928 & 74464.17 & .50 & $\left({ }^{4} S\right) 5 d$ \\
\hline 11 & & 1342.534 & 74486.02 & .33 & $\left.{ }^{2} P\right) 6 p{ }^{3} P_{\mathrm{b}}$ \\
\hline 9 & & 1341.518 & 74542.43 & .53 & $\left({ }^{4} S\right) 5 d{ }^{5} D$ \\
\hline 5 & & 1338.153 & 74729.85 & 30.67 & $\left({ }^{2} D\right) S d{ }^{3} F_{4}$ \\
\hline 4 & & 1337.652 & 74757.88 & .88 & $\left.{ }^{4} S\right) S d{ }^{5} D_{2}$ \\
\hline 11 & & 1333.145 & 75010.60 & .76 & $\left({ }^{2} D\right) 5 d^{3} G_{3}$ \\
\hline 3 & & 1330.927 & 75135.60 & 6.05 & $(S) 5 d{ }^{5} D_{2}$ \\
\hline 1 & & 1327.475 & 75331.01 & .50 & $\left({ }^{4} S\right) S d{ }^{3} D_{1}$ \\
\hline 2 & & 1325.014 & 75470.91 & 1.65 & $5 s 5 p^{5}$ \\
\hline 6 & & 1320.689 & 75718.03 & .61 & $(s) S d d^{s}$ \\
\hline 2 & & 1316.873 & 75937.45 & .63 & $\left({ }^{4} S\right)$ Sd ${ }^{3} D_{1}-\left({ }^{4} S\right) S f{ }^{5} F_{2}$ \\
\hline 8 & & 1316.442 & 75962.30 & .66 & $(S) S d{ }^{5} D_{1}-\left({ }^{2} D\right) 4 f^{3} P_{2}$ \\
\hline 1 & & 1314.390 & 76080.92 & .99 & $\left({ }^{4} S\right) 5 d d^{3} D_{3}-\left({ }^{4} S\right) S f{ }^{5} F_{4}$ \\
\hline 10 & & 1314.088 & 76098.41 & .57 & $\left({ }^{4} S\right) S d{ }^{5} D_{0}-\left({ }^{2} D\right) 4 f^{3} D_{1}$ \\
\hline 4 & $\mathbf{u}$ & 1309.881 & 76342.84 & .62 & $(S) S d{ }^{3} D_{1}-\left({ }^{2} D\right) 4 \int^{3} D_{1}$ \\
\hline 10 & & 1309.818 & 76346.46 & .95 & $5 s^{2} 5 p^{4}{ }^{1} S_{0}-\left({ }^{4} S\right) 5 d{ }^{5} D_{1}$ \\
\hline 2 & & 1309.656 & 76355.92 & 6.24 & $(S) S d{ }^{3} D_{3}-\left({ }^{4} S\right) S f^{5} F_{3}$ \\
\hline
\end{tabular}


Table I. Continued

\begin{tabular}{|c|c|c|c|c|c|}
\hline \multirow{2}{*}{\multicolumn{2}{|c|}{ Intensity' }} & \multirow[b]{2}{*}{$\lambda(\boldsymbol{A})$} & \multicolumn{2}{|l|}{$\sigma\left(\mathrm{cm}^{-1}\right)$} & \multirow[b]{2}{*}{ Classification } \\
\hline & & & obs & calc & \\
\hline 9 & & 1306.260 & 76554.46 & 5.25 & $\left({ }^{2} D\right) 5 d{ }^{3} G_{3}-\left({ }^{2} P\right) 4 f{ }^{\prime} G_{4}$ \\
\hline 4 & & 1305.721 & 76586.02 & .27 & $\left({ }^{2} D\right) 5 d d^{3} F_{4}-\left({ }^{2} P\right) 4 f f^{3} F_{4}$ \\
\hline 0 & & 1305.475 & 76600.49 & .73 & $\left({ }^{2} D\right) S d^{3} G_{4}-\left({ }^{2} P\right) A f \quad{ }^{\prime} D$ \\
\hline 2 & & 1303.385 & 76723.29 & .71 & ('S)Sd ${ }^{3} D_{3}-\left({ }^{2} P\right.$ PAf ${ }^{3} G_{3}$ \\
\hline 2 & & 1299.234 & 76968.45 & .69 & $5 s 5 p^{5} \quad{ }^{3} P_{2}-\left({ }^{2} P\right) 6 p{ }^{3} D_{1}$ \\
\hline 7 & & 1294.672 & 77239.62 & 40.14 & $\left({ }^{2} D\right) 5 d^{3} F_{3}-\left({ }^{2} P\right) 4 f^{3} D_{2}$ \\
\hline 6 & & 1294.477 & 77251.26 & .56 & ('S) $5 d{ }^{3} D_{1}-\left({ }^{2} D\right) 4 f^{3} P_{1}$ \\
\hline 4 & & 1292.427 & 77373.82 & 4.17 & $\left({ }^{4} S\right) 5 d^{S} D_{1}-\left({ }^{2} D\right) 4 f^{\prime} D_{2}$ \\
\hline 11 & & 1281.633 & 78025.43 & .49 & $5 s 5 p^{3} \quad 3 P_{1}-\left({ }^{2} D\right) 4 f$ \\
\hline 2 & & 1278.311 & 78228.25 & .22 & $\left({ }^{2} D\right) 5 d \quad{ }^{1} P_{1}-\left({ }^{2} P\right) 4 f^{3} F_{2}$ \\
\hline 3 & & 1277.742 & 78263.07 & .10 & $\left({ }^{2} D\right) 5 d d^{3} F_{3}-\left({ }^{2} P\right) 4 f^{3} D_{3}$ \\
\hline 10 & & 1272.819 & 78565.77 & .94 & $5 s 5 p^{3}{ }^{3} P_{1}-(-P) 6 p$ \\
\hline 2 & & 1269.293 & 78784.02 & .63 & ( $D) 5 d d^{3} F_{3}-(P) 4 f^{\prime} G_{4}$ \\
\hline 7 & & 1267.171 & 78915.95 & 6.13 & $\left({ }^{4} S\right) S d{ }^{3} D_{2}-\left({ }^{2} P\right) 4 \int^{3} F_{3}$ \\
\hline 10 & & 1253.650 & 79767.06 & 6.86 & $5 s 5 p^{3} \quad{ }^{3} P_{2}-\left(P^{2}\right) 6 p^{3} P_{1}$ \\
\hline 0 & - & 1251.484 & 79905.14 & 4.74 & $5 s 5 p^{3} \quad{ }^{3} P_{1}-\left({ }^{2} D\right) 4 f^{1} P_{1}$ \\
\hline 7 & & 1249.319 & 80043.61 & .61 & $P_{2}-\left({ }^{2} D\right) 4 f$ \\
\hline 3 & & 1247.610 & 80153.27 & .43 & $\left.{ }^{(} S S\right) S d^{3} D_{4}-\left({ }^{\prime} D\right) 4 f^{\prime} G_{4}$ \\
\hline 2 & & 1244.235 & 80370.66 & .92 & $\left({ }^{4} S\right) S d d^{3} D_{2}-\left({ }^{4} S\right) 5 f{ }^{5} F_{1}$ \\
\hline 10 & & 1242.875 & 80458.63 & .76 & $5 s 5 p^{5} \quad{ }^{3} P_{0}-\left({ }^{2} D\right) 4 f^{3} D_{1}$ \\
\hline 1 & & 1238.955 & 80713.18 & .21 & $\left({ }^{4} S\right) S d^{3}$ \\
\hline 2 & $\mathbf{u}$ & 1237.316 & 80820.08 & 19.80 & $D_{3}-\left({ }^{2} D\right) 4 f$ \\
\hline & u & 1232.070 & 81164.24 & 3.74 & $D_{2}-5 s s p^{5}$ \\
\hline 0.5 & & 1228.991 & 81367.53 & .70 & $-(2 D) 4 \int^{3} P_{1}$ \\
\hline 2 & & 1227.943 & 81437.00 & .16 & $\left({ }^{4} S\right) 5 d^{3} D_{1}-\left({ }^{2} P\right) 4 f^{3} D_{2}$ \\
\hline 12 & & 1205.929 & 82923.60 & .08 & $\left.5 s^{2} 5 p^{4} S_{0}-{ }^{2} D\right) 5 d^{1} P_{i}$ \\
\hline 2 & & 1202.593 & 83153.65 & .29 & $\left({ }^{4} S\right) 5 d^{3} D_{3}-\left({ }^{7} P\right) 4 f^{3} D_{3}$ \\
\hline 4 & & 1200.033 & 83331.02 & .23 & $555 p^{5} \quad{ }^{3} P_{2}-\left({ }^{2} D\right) 4 f^{3} F_{2}$ \\
\hline 11 & & 1198.683 & 83424.86 & .22 & $-\left({ }^{2} D\right) 4 f^{3} D_{2}$ \\
\hline 2 & & 1195.107 & 83674.55 & .82 & ${ }_{3}-\left({ }^{2} P\right) 4 f^{\prime}$ \\
\hline 11 & & 1188.853 & 84114.69 & .54 & $5 s 5 p^{5} \quad{ }^{3} P_{2}-\left({ }^{2} D\right) 4 f^{3}$ \\
\hline 1 & & 1186.249 & 84299.35 & .83 & $\left.\left({ }^{4} S\right) S d d^{5} D_{2}-\left({ }^{2} P\right) 4 f^{3}\right]$ \\
\hline 2 & & 1185.767 & 84333.60 & .88 & (2D)Sd ${ }^{1} P_{1}-\left({ }^{2} P\right) 4 f^{3} D_{2}$ \\
\hline 1 & & 1182.729 & 84550.21 & .80 & $\left({ }^{4} S\right) S d^{5} D_{3}-\left({ }^{2} P\right) 4 f^{3}$ \\
\hline 4 & & 1179.186 & 84804.29 & .35 & $\left({ }^{4} S\right) S d{ }^{3} D_{1}-\left({ }^{2} P\right) 4 f$ \\
\hline 8 & & 1178.630 & 84844.30 & .36 & $-\left({ }^{2} D\right) 4 f^{3}$ \\
\hline 1 & & 1177.617 & 84917.28 & .05 & $\left({ }^{4} S\right) 5 d d^{5} D_{0}-\left({ }^{4} S\right) 5 f^{5} F_{1}$ \\
\hline 2 & & 1174.243 & 85161.27 & .10 & ('s) $5 d d^{5} D_{1}-\left({ }^{4} s\right) 5 f^{s} F_{1}$ \\
\hline 7 & & 1173.857 & 85189.24 & 8.89 & $\left({ }^{4} S\right) S d^{5} D_{4}-\left({ }^{4} S\right) S f^{5}$ \\
\hline 0.5 & & 1173.370 & 85224.64 & .31 & ${ }_{5}^{5} 5 p^{5} \quad{ }^{3} P_{1}-\left({ }^{2} D\right) 4 f^{3} 1$ \\
\hline 9 & & 1173.146 & 85240.89 & .43 & $\left.{ }^{4} s\right)^{6} s^{3} s_{1}-5 s^{0} 5 p^{6} \quad s_{0}$ \\
\hline 2 & & 1170.988 & 85397.97 & .87 & $\left.{ }^{4} S\right) 5 d d^{5} D_{2}-\left({ }^{2} P\right) 4 f^{3}{ }^{3} F_{2}$ \\
\hline 2 & & 1170.812 & 85410.79 & .48 & $\left({ }^{4} S\right) S d^{5} D_{1}-\left({ }^{4} S\right) S f f^{5}$ \\
\hline 9 & & 1166.789 & 85705.29 & .16 & $\left({ }^{4} S\right) S d^{3} D_{3}-\left({ }^{4} S\right) S \int^{5} F_{4}$ \\
\hline 8 & & 1166.467 & 85728.92 & 9.44 & $\left({ }^{4} S\right) S d d^{5} D_{2}-\left({ }^{4} S\right) S f^{S} F_{3}$ \\
\hline 0.5 & & 1166.228 & 85746.53 & .63 & $555 p^{5}{ }^{3} P_{2}-\left({ }^{2} P\right) 6 p p^{1} D_{2}$ \\
\hline 1 & & 1163.061 & 85980.05 & .41 & ('S) $5 d d^{5} D_{3}-\left({ }^{4} S\right) S f$ \\
\hline 2 & & 1160.992 & 86133.25 & .26 & $5 s 5 p^{5} \quad{ }^{3} p_{1}-\left({ }^{2} D\right) 4 f$ \\
\hline 10 & & 1158.329 & 86331.26 & .98 & $5 s 5 p^{5} \quad{ }^{3} P_{2}-\left({ }^{2} P\right) 6 p$ \\
\hline 11 & & 1156.475 & 86469.69 & .47 & $5 s^{2} 5 p^{4} \quad D_{2}-5 s 5 p^{3}$ \\
\hline 7 & & 1135.613 & 88058.15 & .41 & $5 s 5 p^{3} \quad{ }^{3} P_{2}-\left({ }^{2} P\right) 6 p$ \\
\hline 0 & & 1131.838 & 88351.89 & .79 & ${ }^{3} P_{2}-\left({ }^{2} D\right) 4 f$ \\
\hline 12 & & 1130.348 & 88468.32 & .11 & $5 s^{2} 5 p^{4}{ }^{3} P_{1}-5 s 5 p^{5}{ }^{3} P_{2}$ \\
\hline 2 & & 1127.018 & 88729.74 & .96 & $555 p^{3} \quad{ }^{3} P_{2}-\left({ }^{2} D\right) 4 f^{3} D_{2}$ \\
\hline 7 & & 1124.428 & 88934.09 & .74 & $\left({ }^{4} S\right) S d^{3} D_{1}-5 s^{0} 5 p^{6}{ }^{1} S_{0}$ \\
\hline 10 & & 1109.257 & 90150.46 & .09 & $585 p^{3} \quad{ }^{3} P_{2}-\left({ }^{2} D\right) 4 f^{3} P_{2}$ \\
\hline 10 & & 1092.168 & 91561.03 & .60 & $5 s 5 p^{5} \quad{ }^{3} P_{2}-\left({ }^{2} D\right) 4 f^{1} D_{2}$ \\
\hline 20 & & 1088.954 & 91831.25 & .46 & $\left.{ }^{2} D\right) 5 d^{1} P_{1}-5 s^{0} 5 p^{6}{ }^{1} S_{0}$ \\
\hline 1 & & 1077.844 & 92777.81 & .46 & ('S $S) 5 d^{5} D_{3}-\left({ }^{2} P\right) 4 f^{3} D_{3}$ \\
\hline 18 & & 1066.393 & 93774.02 & 3.85 & $5 s^{2} 5 p^{4}{ }^{3} P_{1}-5 s 5 p^{5} \quad{ }^{3} P_{1}$ \\
\hline 1 & & 1060.529 & 94292.61 & .18 & $5 s 5 p^{5} \quad{ }^{3} P_{1}-\left({ }^{4} S\right) 5 f{ }^{5} F_{2}$ \\
\hline 9 & & 1058.136 & 94505.83 & 6.68 & $5 s^{2} 5 p^{4} D_{2}-\left({ }^{4} S\right) 5 d^{5} D_{3}$ \\
\hline 11 & & 1055.326 & 94757.44 & .65 & $5 s^{2} 5 p^{4}{ }^{1} D_{2}-\left({ }^{4} S\right) S d^{5} D_{2}$ \\
\hline 11 & & 1048.755 & 95351.18 & .16 & $5 s^{2} 5 p^{4} D_{2}-(4 S) 5 d^{5} D_{1}$ \\
\hline 15 & & 1047.799 & 95438.18 & .12 & $5 s^{2} 5 p^{4}{ }^{3} P_{0}-5 s 5 p^{5}{ }^{3} P_{1}$ \\
\hline 13 & & 1017.682 & 98262.53 & .47 & $5 s^{2} 5 p^{4}{ }^{3} P_{2}-5 s 5 p^{3} \quad{ }^{3} P_{2}$ \\
\hline 2 & & 1016.188 & 98406.97 & 7.59 & $\left({ }^{4} S\right) S d^{3} D_{1}-5 s^{0} 5 p^{6} \quad S_{0}$ \\
\hline 12 & & 1014.825 & 98539.15 & .40 & $5 s^{2} 5 p^{4}{ }^{3} P_{1}-5 s 5 p^{3} \quad{ }^{3} P_{0}$ \\
\hline
\end{tabular}

Table I. Continued

\begin{tabular}{|c|c|c|c|c|}
\hline \multirow[b]{2}{*}{ Intensity' } & \multirow[b]{2}{*}{$\lambda(A)$} & \multicolumn{2}{|l|}{$\sigma\left(\mathrm{cm}^{-1}\right)$} & \multirow[b]{2}{*}{ Classification } \\
\hline & & obs & calc & \\
\hline 6 & 1002.087 & 99791.73 & .71 & $5 s 5 p^{5}{ }^{3} P_{1}-\left({ }^{2} P\right) 4 f^{3} D$ \\
\hline 12 & 981.097 & 101926.8 & 7.29 & $55^{2} 5 p^{4} \quad D_{2}-\left({ }^{2} D\right) 5 d^{\prime} P$ \\
\hline (1) & 979.980 & 102043. & 2.55 & $S_{0}-\left({ }^{2} D\right) 65^{3} D$ \\
\hline 12 & 974.133 & 102655.4 & .54 & $5 s^{2} 5 p^{4}{ }^{3} P_{1}-\left({ }^{4} S\right) S d^{5} D$ \\
\hline 15 & 971.818 & 102899.9 & .59 & $55^{2} 5 p^{4}{ }^{3} P_{1}-\left({ }^{4} S\right) 5 d^{5} D_{0}$ \\
\hline 10 & 965.548 & 103568.2 & .20 & $5 s^{2} 5 p^{4} 3^{3} P_{2}-5 s 5 p^{5} \quad{ }^{3} P_{1}$ \\
\hline (1) & 960.325 & 104131. & 0.85 & $D_{2}-\left({ }^{4} S\right) 5 d$ \\
\hline 10 & 958.591 & 104319.8 & .82 & $5 s^{2} S p^{4}{ }^{3} P_{0}-\left(^{4} S\right) 5 d^{3} D$ \\
\hline 11 & 953.983 & 104823.7 & 4.02 & ${ }^{\prime} D_{2}-\left({ }^{4} S\right) S d^{\prime} D_{1}$ \\
\hline 2 & 930.702 & 107445.7 & .72 & $5 s^{2} 5 p^{4}{ }^{3} P_{1}-\left({ }^{4} S\right) 5 d^{3} D$ \\
\hline 1 & 917.258 & 109020.6 & 1.04 & $S 5^{2} S p^{4} \quad D_{2}-\left({ }^{2} D\right) S d^{3} F_{3}$ \\
\hline 10 & 915.487 & 109231.5 & .67 & $-\left({ }^{2} D\right) S d$ ' $P$ \\
\hline 13 & 901.745 & 110896.1 & 5.95 & $P_{0}-\left({ }^{2} D\right) S d$ \\
\hline 7 & 898.870 & 111250.8 & .42 & $D_{2}-\left({ }^{2} D\right) S d{ }^{3} G$ \\
\hline 13 & 896.014 & 111605.4 & .41 & $D_{2}-\left({ }^{4} S\right) S d^{3} D$ \\
\hline 6 & 895.401 & 111681.8 & .58 & $-(4 S) 6 s$ \\
\hline 13 & 894.003 & 111856.5 & .38 & $P_{2}-\left({ }^{4} S\right) 5 d d^{5} D_{2}$ \\
\hline 11 & 891.835 & 112128.4 & .39 & $P_{1}-\left({ }^{4} S\right) S d^{3} D$ \\
\hline 13 & 889.284 & 112450.0 & 49.90 & $P_{2}-\left({ }^{4} S\right) 5 d^{5}$ \\
\hline 10 & 878.789 & 113793.0 & 2.67 & $-\left({ }^{4} s\right) 5 d^{3}$ \\
\hline 2 & 870.342 & 114897.4 & 6.97 & $-\left({ }^{2} D\right) 5 d^{3}$ \\
\hline 1 & 863.385 & 115823.2 & 2.70 & $-\left({ }^{4} S\right) 6 s^{3} S_{1}$ \\
\hline 6 & 861.064 & 116135.4 & .28 & $-\left({ }^{2} D\right) S d d^{3} D$ \\
\hline 13 & 852.947 & 117240.6 & .08 & $2-\left({ }^{4} S\right) 5 d^{3}$ \\
\hline 2 & 851.152 & 117487.8 & 6.98 & $0-(4 S) 6 s^{3}$ \\
\hline 3 & 850.563 & 117569.2 & 8.68 & $5 s^{2} 5 p^{4}$ \\
\hline 11 & 840.151 & 119026.2 & .03 & $P_{2}-\left({ }^{2} D\right) 5 d$ \\
\hline 0 & 838.441 & 119269.0 & 8.74 & $D_{2}-\left({ }^{2} P\right) 5 d$ \\
\hline (1) & 838.244 & 119297. & .96 & $S_{0}-\left({ }^{2} P\right) S d^{3} D$ \\
\hline (3) & 826.132 & 121046 & .76 & $D_{2}-\left({ }^{2} D\right) 6 s^{3} D_{1}$ \\
\hline 12 & 824.878 & 121230.1 & 29.58 & $P_{2}-\left({ }^{4} S\right) 5 d^{3}$ \\
\hline 15 & 823.202 & 121476.9 & 5.94 & $2-\left({ }^{4} s\right) 6 s^{3}$ \\
\hline 8 & 822.640 & 121559.8 & .47 & $b_{2}-\left(^{2} D\right) 6 s^{3}$ \\
\hline (4) & 820.166 & 121926. & 2.75 & $P_{2}-\left({ }^{4} S\right) 5 d^{3} D$ \\
\hline (2) & 811.138 & 123284 & 5.23 & $S_{0}-\left({ }^{2} P\right) 6 s \quad{ }^{1} P_{1}$ \\
\hline 10 & 810.110 & 123440.0 & 39.66 & $P_{1}-\left({ }^{2} D\right) 5 d^{3} D$ \\
\hline (2) & 808.860 & 123631. & 2.20 & $D_{2}-\left({ }^{2} P\right) 5 d d^{3} P_{1}$ \\
\hline i1 & 801.978 & 124691.6 & .33 & $P_{2}-\left({ }^{2} D\right) S d^{3} F_{2}$ \\
\hline 2 & 800.835 & 124869.6 & 73.06 & $P_{1}-\left({ }^{2} D\right) 6 s^{3} D$ \\
\hline (0) & 800.228 & 124964. & 5.53 & $D_{2}-\left({ }^{2} D\right) S d^{3} D$ \\
\hline 11 & 799.333 & 125104.2 & 3.93 & $P_{0}-\left(^{2} D\right) S d^{3} D$ \\
\hline 11 & 796.067 & 125617.5 & .06 & $P_{2}-\left({ }^{\prime} S\right) 6 s^{3} S_{1}$ \\
\hline 9 & 793.968 & 125949.6 & .47 & $D_{2}-\left({ }^{2} D\right) 6 s s^{\prime} D$ \\
\hline 0 & 793.282 & 126058.5 & 7.51 & $D_{2}-\left({ }^{2} D\right) 5 d^{3} D$ \\
\hline 12 & 792.896 & 126120.0 & 19.77 & $P_{2}-\left({ }^{2} D\right) S d^{3} F_{3}$ \\
\hline 4 & 790.056 & 126573.2 & .12 & $P_{1}-\left({ }^{2} P\right) 5 d d^{\prime} D$ \\
\hline (1) & 784.785 & 127423. & 4.46 & $5 s^{2} 5 p^{4}$ \\
\hline 8 & 780.027 & 128200.6 & 1.40 & $-\left({ }^{2} P\right) 5 d$ \\
\hline (4) & 779.782 & 128241 & 2.18 & $55^{2} 5 p^{4}$ \\
\hline 13 & 779.124 & 128349.3 & .15 & $2-\left({ }^{2} D\right) 5 d^{3} G$ \\
\hline 13 & 779.124 & 128349.3 & 51.13 & $5 s^{2} 5 p^{4}{ }^{3} P_{1}-\left({ }^{2} D\right) 6 s^{3} D$ \\
\hline 8 & 769.140 & 130015.3 & .41 & $5 s^{2} 5 p^{4}{ }^{3} P_{0}-\left({ }^{2} D\right) 6 s^{3} D$ \\
\hline 2 & 765.442 & 130643.5 & .43 & $55^{2} 5 p^{4}{ }^{3} P_{1}-\left({ }^{2} P\right) 5 d d^{3} P_{0}$ \\
\hline (7) & 765.120 & 130698. & .68 & $5 s^{2} 5 p^{4} \quad D_{2}-\left({ }^{2} D\right) 5 d^{3} S_{1}$ \\
\hline 9 & 763.729 & 130936.5 & .57 & $55^{2} 5 p^{4}{ }^{3} P_{1}-\left({ }^{2} P\right) 5 d d^{3} P_{1}$ \\
\hline (5) & 761.790 & 131270 & 1.40 & $5 s^{2} 5 p^{4} D_{2}-\left({ }^{2} D\right) S d^{3} P_{2}$ \\
\hline 1 & 761.532 & 131314.2 & .10 & $55^{2} 5 p^{4}{ }^{1} D_{2}-\left({ }^{2} D\right) 5 d^{\prime} F_{3}$ \\
\hline 6 & 756.031 & 132269.7 & .91 & $5 s^{2} 5 p^{4}{ }^{3} P_{1}-\left({ }^{2} D\right) 5 d^{3} D$ \\
\hline (6) & 754.144 & 132601 & 0.85 & $55^{2} 5 p^{4}$ \\
\hline 5 & 750.451 & 133253.2 & .84 & $5 s^{2} 5 p^{4}{ }^{3} P_{1}-\left({ }^{2} D\right) 6 s^{1} D$ \\
\hline 4 & 750.160 & 133305.0 & .51 & $5 s^{2} 5 p^{4} \quad D_{2}-\left({ }^{2} P\right) S d^{3} P_{2}$ \\
\hline 2 & 744.142 & 134382.9 & $\mathbf{3 . 7 0}$ & $5 s^{2} 5 p^{4}{ }^{1} D_{2}-\left(^{2} P\right) 6 s^{3}$ \\
\hline 13 & 742.570 & 134667.5 & .42 & $55^{2} 5 p^{4}{ }^{3} P_{2}-\left({ }^{2} D\right) 6 s^{3}$ \\
\hline 7 & 737.977 & 135505.6 & .77 & $5 s^{2} 5 p^{4}{ }^{3} P_{1}-\left({ }^{2} P\right) 5 d^{3} F_{2}$ \\
\hline 12 & 733.314 & 136367.3 & .48 & $55^{2} 5 p^{4}{ }^{3} P_{2}-\left({ }^{2} P\right) 5 d^{1} D$ \\
\hline 5 & 731.023 & 136794.6 & .47 & $5 s^{2} 5 p^{4} \quad D_{2}-\left({ }^{2} P\right) S d^{3} D$ \\
\hline 12 & 727.042 & 137543.6 & 0.64 & $S s^{2} 5 p^{4} D_{2}-\left({ }^{2} D\right) S d^{3}$ \\
\hline (1) & 724.623 & 138003 & .06 & $P_{1}-\left({ }^{2} D\right) 5 d^{3}$ \\
\hline
\end{tabular}


Table I. Continued

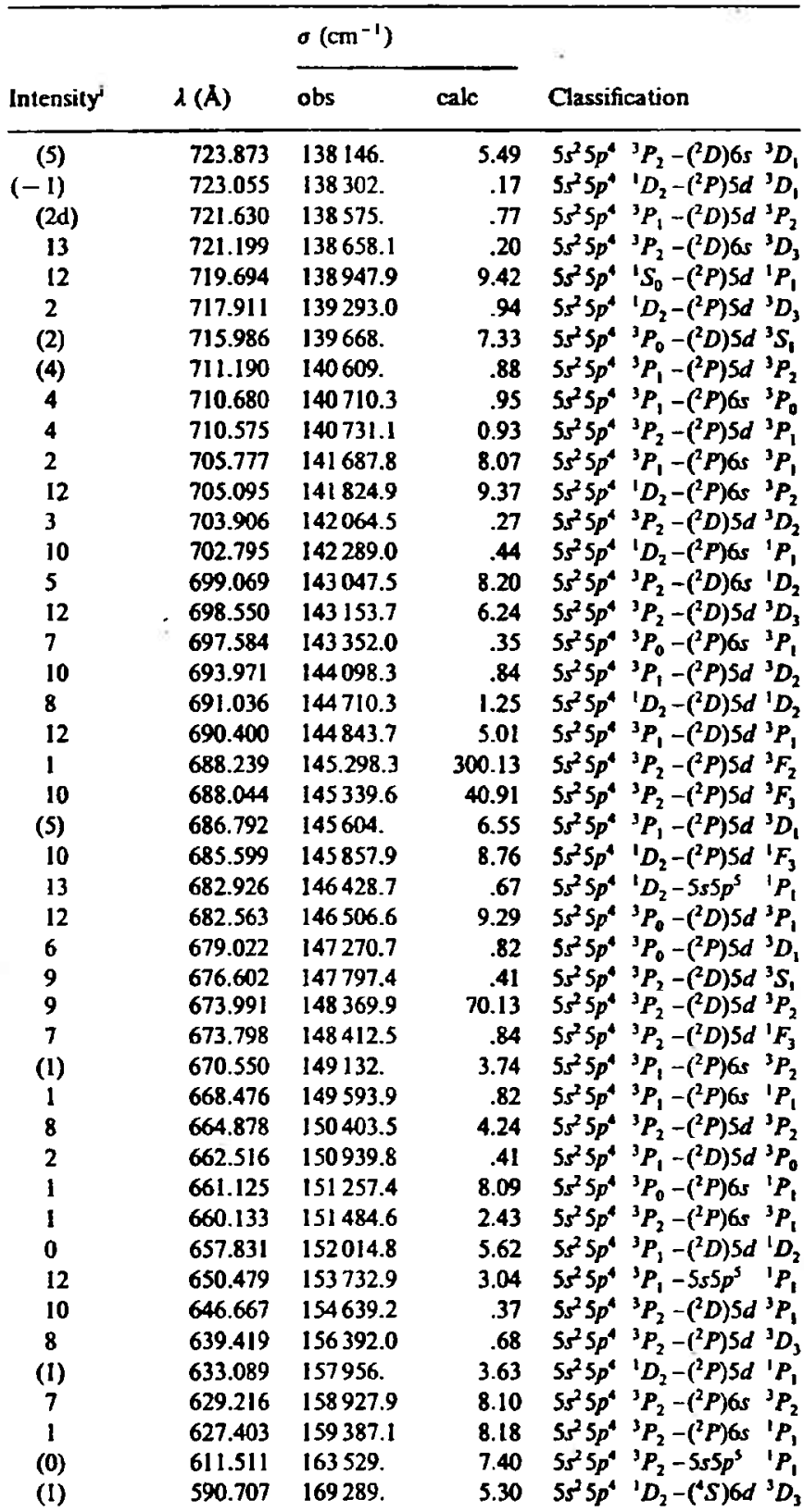

Table I. Continued

$\sigma\left(\mathrm{cm}^{-1}\right)$

Intensity $\lambda(A)$ obs calc Classification

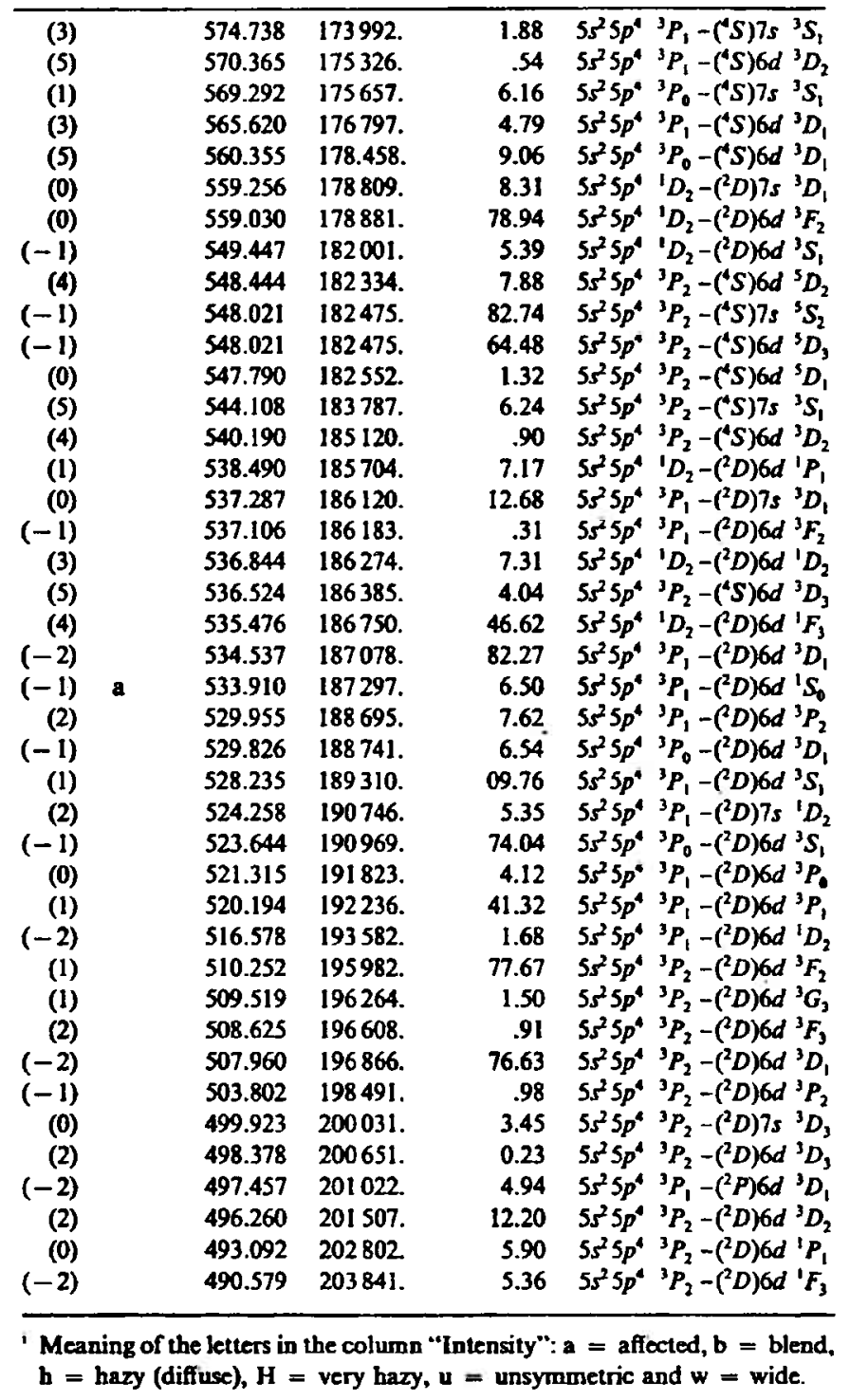


Table II. Even levels of Xe III. All levels are given in LS Table II. Continued notation. The numbers in parentheses indicate the purities (in \%) of the states

\begin{tabular}{|c|c|}
\hline Designation & \multirow{2}{*}{$\begin{array}{r}E\left(\mathrm{~cm}^{-1}\right) \\
0.00(86) \\
9794.36(98) \\
8130.08(79)\end{array}$} \\
\hline $\begin{array}{r}55^{2} 5 p^{4}{ }^{3} P_{2} \\
{ }^{3} P_{1} \\
{ }^{3} P_{0}\end{array}$ & \\
\hline${ }^{\prime} D_{2}$ & $17098.73(86)$ \\
\hline 'S $S_{0}$ & $36102.94(78)$ \\
\hline $\begin{array}{r}55^{2} 5 p^{3}(C S) 6 p{ }^{s} P_{3} \\
{ }^{s} P_{2} \\
{ }^{s} P_{1}\end{array}$ & $\begin{array}{l}149061.57(84) \\
146962.42(66) \\
146781.48(73)\end{array}$ \\
\hline $\begin{array}{l}{ }^{3} P_{2} \\
{ }^{3} P_{1} \\
{ }^{3} P_{0}\end{array}$ & $\begin{array}{l}152057.72(64) \\
150301.10(57) \\
152808.17(89)\end{array}$ \\
\hline $\begin{array}{r}\left.{ }^{2} D\right) 6 p{ }^{3} F_{4} \\
={ }^{3} F_{3} \\
{ }^{3} F_{2}\end{array}$ & $\begin{array}{l}166554.82(82) \\
162594.81(56) \\
160691.30(54)\end{array}$ \\
\hline $\begin{array}{l}{ }^{3} D_{3} \\
{ }^{3} D_{2} \\
{ }^{3} D_{1}\end{array}$ & $\begin{array}{l}166699.11(66) \\
162259.97(47) \\
158996.98(38)\end{array}$ \\
\hline $\begin{array}{l}{ }^{3} P_{2} \\
{ }^{3} P_{1} \\
{ }^{3} P_{0}\end{array}$ & $\begin{array}{l}167066.32(55) \\
168086.00(62) \\
165941.69(84)\end{array}$ \\
\hline${ }^{\prime} F_{3}$ & $164438.64(49)$ \\
\hline${ }^{\prime} D_{2}$ & $171989.82(70)$ \\
\hline${ }^{\prime} P_{1}$ & $164511.65(41)$ \\
\hline $\begin{array}{r}\left.{ }^{2} P\right) 6 P \\
{ }^{3} D_{3} \\
{ }^{3} D_{2} \\
{ }^{3} D_{1}\end{array}$ & $\begin{array}{l}184594.45(57) \\
177955.93(59) \\
175231.15(66)\end{array}$ \\
\hline $\begin{array}{l}{ }^{3} P_{2} \\
{ }^{3} P_{1} \\
{ }^{3} P_{0}\end{array}$ & $\begin{array}{l}186320.88(27) \\
178029.33(59) \\
178054.53(83)\end{array}$ \\
\hline${ }^{3} S_{1}$ & $182134.14(58)$ \\
\hline${ }^{\prime} D_{2}$ & $184009.10(44)$ \\
\hline${ }^{\prime} P_{1}$ & 185888.03 \\
\hline 'S & $190491.16(67)$ \\
\hline $\begin{array}{r}5 s^{2} 5 p^{3}\left({ }^{4} S\right) 4 f^{s} F_{3} \\
{ }^{s} F_{4} \\
{ }^{3} F_{3}\end{array}$ & $\begin{array}{l}166743.80(84) \\
166355.27(64) \\
166374.06(74)\end{array}$ \\
\hline
\end{tabular}

\begin{tabular}{|c|c|}
\hline Designation & $E\left(\mathrm{~cm}^{-1}\right)$ \\
\hline $\begin{array}{l}{ }^{s} F_{2} \\
{ }^{s} F_{1}\end{array}$ & $\begin{array}{l}166880.09(81) \\
167173.54(87)\end{array}$ \\
\hline $\begin{array}{l}{ }^{3} F_{4} \\
{ }^{3} F_{3} \\
{ }^{3} F_{2}\end{array}$ & $\begin{array}{l}173946.53(65) \\
170250.15(42) \\
173734.12(77)\end{array}$ \\
\hline $\begin{array}{r}\left.{ }^{2} D\right) 4 \int^{3} H_{6} \\
{ }^{3} H_{3} \\
{ }^{3} H_{4}\end{array}$ & $\begin{array}{l}186086.52(100 \\
181356.80(36) \\
181684.94(56)\end{array}$ \\
\hline $\begin{array}{l}{ }^{3} G_{3} \\
{ }^{3} G_{4} \\
{ }^{3} G_{3}\end{array}$ & $\begin{array}{l}184114.53(81) \\
178887.17(58) \\
178306.08(51)\end{array}$ \\
\hline $\begin{array}{l}{ }^{3} F_{4} \\
{ }^{3} F_{3} \\
{ }^{3} F_{2}\end{array}$ & $\begin{array}{l}185406.74(42) \\
186614.26(67) \\
181593.70(41)\end{array}$ \\
\hline $\begin{array}{l}{ }^{3} D_{3} \\
{ }^{3} D_{2} \\
{ }^{3} D_{1}\end{array}$ & $\begin{array}{l}182377.01(42) \\
186992.43(25) \\
188792.52(92)\end{array}$ \\
\hline $\begin{array}{l}{ }^{3} P_{2} \\
{ }^{3} P_{1} \\
{ }^{3} P_{0}\end{array}$ & $\begin{array}{l}188412.56(38) \\
189701.46(79)\end{array}$ \\
\hline ' $H_{5}$ & $186022.92(62)$ \\
\hline$\quad G_{4}$ & $192425.21(76)$ \\
\hline${ }^{\prime} F_{\mathbf{3}}$ & $189778.94(55)$ \\
\hline$' D_{2}$ & $189824.07(50)$ \\
\hline${ }^{1} P_{1}$ & $183472.95(70)$ \\
\hline $\begin{array}{r}\left({ }^{2} P\right) 4 f^{3} G_{3} \\
{ }^{3} F_{4} \\
{ }^{3} F_{3} \\
{ }^{3} F_{2}\end{array}$ & $\begin{array}{l}197953.29(29) \\
206760.00(30) \\
196156.21(51) \\
197254.25(43)\end{array}$ \\
\hline $\begin{array}{l}{ }^{3} D_{3} \\
{ }^{3} D_{2} \\
{ }^{3} D_{1}\end{array}$ & $\begin{array}{l}204382.87(43) \\
203359.91(51)\end{array}$ \\
\hline ' $G$ & 204904.40 (34) \\
\hline $\begin{array}{r}5 s^{2} 5 p^{3}\left({ }^{4} S\right) S f{ }^{5} F_{3} \\
{ }^{5} F_{4} \\
{ }^{5} F_{3} \\
{ }^{5} F_{2} \\
{ }^{5} F_{1}\end{array}$ & $\begin{array}{l}197460.67(89) \\
197310.57(74) \\
197585.82(56) \\
197860.38(68) \\
197611.00(90)\end{array}$ \\
\hline $5 s^{0} 5 p^{6}{ }^{1} S_{0}$ & $210857.49(56)$ \\
\hline
\end{tabular}


Table III. Odd levels of Xe III. All levels are given in LS Table III. Continued notation. The numbers in parentheses indicate the purities (in \%) of the states

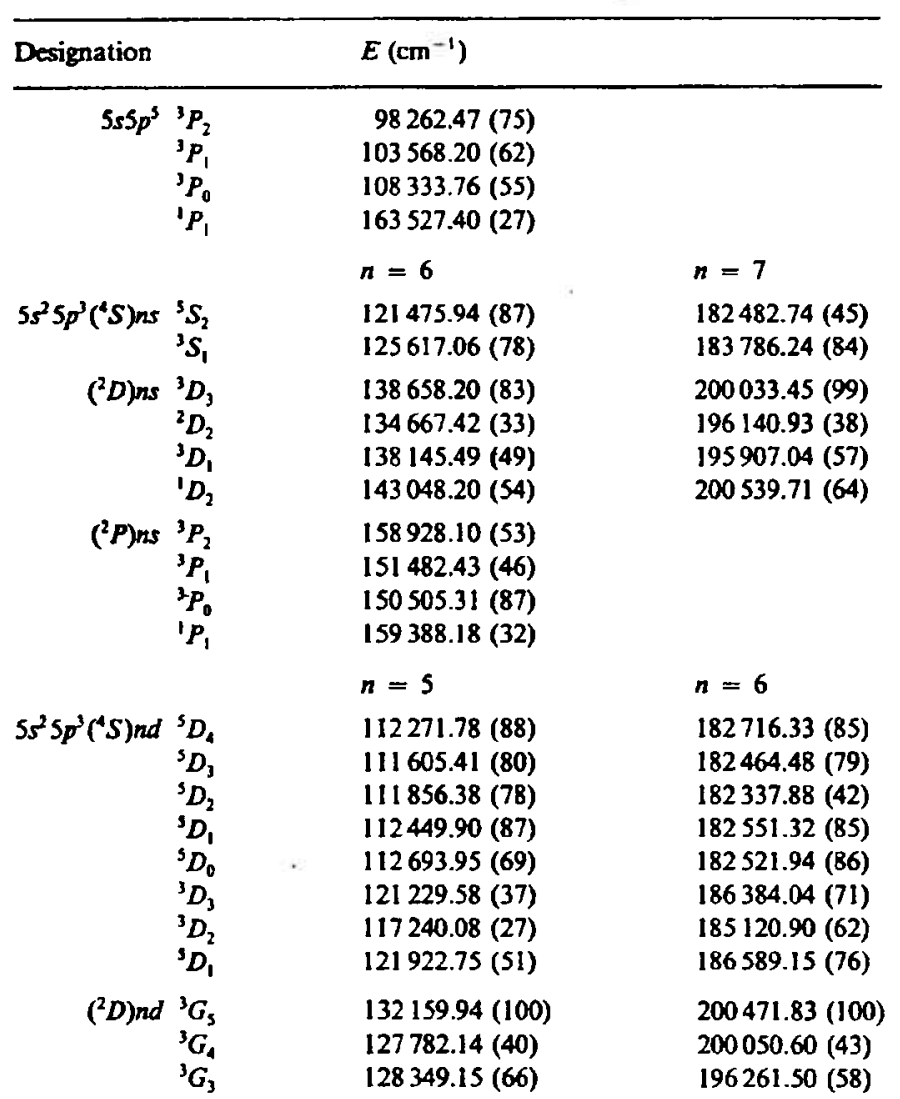

\begin{tabular}{|c|c|c|c|}
\hline \multicolumn{2}{|l|}{ Designation } & \multicolumn{2}{|l|}{$E\left(\mathrm{~cm}^{-1}\right)$} \\
\hline & 'G. & $132711.78(73)$ & $196538.07(31)$ \\
\hline & $\begin{array}{l}{ }^{3} F_{4} \\
{ }^{3} F_{3} \\
{ }^{3} F_{2}\end{array}$ & $\begin{array}{l}130173.73(71) \\
126119.77(58) \\
124691.33(52)\end{array}$ & $\begin{array}{l}200425.68(82) \\
196608.91(43) \\
195977.67(47)\end{array}$ \\
\hline & ${ }^{\prime} F_{3}$ & $148412.84(42)$ & $203845.36(71)$ \\
\hline & $\begin{array}{l}{ }^{3} D_{3} \\
{ }^{3} D_{2} \\
{ }^{3} D_{1}\end{array}$ & $\begin{array}{l}143156.24(38) \\
142064.27(24) \\
133234.01(36)\end{array}$ & $\begin{array}{l}200650.23(64) \\
201512.20(44) \\
196876.63(45)\end{array}$ \\
\hline & ${ }^{\prime} D_{2}$ & $161809.98(39)$ & $203376.04(54)$ \\
\hline & $\begin{array}{l}{ }^{3} P_{2} \\
{ }^{3} P_{1} \\
{ }^{3} P_{0}\end{array}$ & $\begin{array}{l}148370.13(39) \\
154639.37(20) \\
160733.77(43)\end{array}$ & $\begin{array}{l}198491.98(22) \\
202035.68(46) \\
201618.48(62)\end{array}$ \\
\hline & ${ }^{3} P_{1}$ & $119026.03(44)$ & $202805.90(37)$ \\
\hline & ${ }^{3} S_{1}$ & $147797.41(49)$ & $199104.12(45)$ \\
\hline & ${ }^{\prime} S_{0}$ & & $197090.86(58)$ \\
\hline$\left({ }^{2} P\right) n d$ & $\begin{array}{l}{ }^{3} F_{4} \\
{ }^{3} F_{3} \\
{ }^{3} F_{2}\end{array}$ & $\begin{array}{l}148535.52(70) \\
145340.91(56) \\
145300.13(50)\end{array}$ & \\
\hline & ${ }^{3} F_{3}$ & $162957.50(40)$ & \\
\hline & $\begin{array}{l}{ }^{3} D_{3} \\
{ }^{3} D_{2} \\
{ }^{3} D_{1}\end{array}$ & $\begin{array}{l}156392.68(33) \\
153893.20(28) \\
155400.90(45)\end{array}$ & \\
\hline & ${ }^{\prime} D_{2}$ & $136367.48(15)$ & \\
\hline & $\begin{array}{l}{ }^{3} P_{2} \\
{ }^{3} P_{1} \\
{ }^{3} P_{0}\end{array}$ & $\begin{array}{l}150404.24(54) \\
140730.93(44) \\
140437.79(42)\end{array}$ & \\
\hline & ${ }^{1} P_{1}$ & $175052.36(64)$ & \\
\hline
\end{tabular}


Table IV. Comparison between observed and calculated energy-level values (in $\mathrm{cm}^{-1}$ ) and calculated percentage compositions for the $5 s^{2} 5 p^{4}+5 s^{2} 5 p^{3}(6 p+4 f+5 f)+5 s^{0} 5 p^{6}+\left(5 s 5 p^{4} 5 d\right)^{2}$ configurations of Xe III. Eigenvector components larger than $5 \%$ are given. Observed [3] and calculated $g$, factors are listed

\begin{tabular}{|c|c|c|c|c|c|c|}
\hline$J$ & $E$ (obs) & $E$ (calc) & obs - calc & $g_{j}($ obs) & $g($ calc) & Percentage composition \\
\hline 6 & 186087 & 185871 & 215 & & 1.167 & $100\left({ }^{2} D\right) 4 f^{3} H$ \\
\hline \multirow[t]{5}{*}{5} & 166744 & 166592 & 152 & & 1.37 & $84\left({ }^{4} S\right) 4 f^{3} F+11\left({ }^{2} P\right) 4 f^{3} G$ \\
\hline & 181357 & 181345 & 12 & & 1.11 & $36\left({ }^{2} D\right) 4 f^{3} H+22\left({ }^{2} D\right) 4 f^{1} H+17\left({ }^{2} P\right) 4 f^{3} G+15\left({ }^{2} D\right) 4 f^{3} G+9\left({ }^{4} S\right) 4 f^{5} F$ \\
\hline & 184115 & 184477 & -363 & & 1.18 & $81\left({ }^{2} D\right) 4 f^{3} G+13\left({ }^{3} D\right) 4 f^{3} H$ \\
\hline & 186023 & 186324 & -301 & & 1.01 & $62\left({ }^{2} D\right) 4 f^{\prime} H+38\left({ }^{2} D\right) 4 f^{3} H$ \\
\hline & 197461 & 197583 & -123 & & 1.38 & $89\left({ }^{4} S\right) S f^{5} F+7\left({ }^{2} P\right) S f^{3} G$ \\
\hline \multirow[t]{10}{*}{4} & 166355 & 166084 & 271 & & 1.30 & $64\left({ }^{4} S\right) 4 f^{3} F+17\left({ }^{2} D\right) 6 p^{3} F+7\left({ }^{2} P\right) 4 f^{3} F+5\left({ }^{2} P\right) 4 f^{3} G$ \\
\hline & 166555 & 166999 & -444 & & 1.26 & $82\left({ }^{2} D\right) 6 p^{3} F+13\left({ }^{4} S\right) 4 f^{3} F$ \\
\hline & 173947 & 174182 & -235 & & 1.21 & $65\left({ }^{4} S\right) 4 f^{3} F+9\left({ }^{2} D\right) 4 f^{3} F+8\left({ }^{4} S\right) 4 f^{3} F+6\left({ }^{2} D\right) 4 f^{3} H+6\left({ }^{2} P\right) 4 f^{\prime} G$ \\
\hline & 178887 & 179265 & -378 & & 1.11 & $\begin{array}{l}58\left({ }^{2} D\right) 4 f^{3} G+9\left({ }^{4} S\right) 4 f^{5} F+6\left({ }^{2} P\right) 4 f^{3} G+6\left({ }^{2} P\right) 4 f^{\prime} G+6\left({ }^{4} S\right) 4 f^{3} F+ \\
6\left({ }^{2} P\right) 4 f^{3} F+5\left({ }^{2} D\right) 4 f^{3} F\end{array}$ \\
\hline & 181685 & 181834 & -149 & & 0.96 & $56\left({ }^{2} D\right) 4 f^{3} H+23\left({ }^{2} D\right) 4 f^{3} F+8\left({ }^{2} P\right) 4 f^{3} G$ \\
\hline & 185407 & 185544 & -137 & & 1.14 & $42\left({ }^{2} D\right) 4 f^{3} F+20\left({ }^{2} D\right) 4 f^{3} H+16\left({ }^{2} D\right) 4 f^{3} G+12\left({ }^{4} S\right) 4 f^{3} F$ \\
\hline & 192425 & 192122 & 303 & & 1.03 & $76\left({ }^{2} D\right) 4 f^{\prime} G+6\left({ }^{2} D\right) 4 f^{3} G+5\left({ }^{2} D\right) 4 f^{3} F$ \\
\hline & 197311 & 197619 & -309 & & 1.31 & $74\left({ }^{4} S\right) S f^{5} F+8\left({ }^{2} P\right) 4 f^{3} G$ \\
\hline & 204904 & 205178 & -273 & & 1.06 & $34\left({ }^{2} P\right) 4 f^{1} G+17\left({ }^{2} P\right) 4 f^{3} G+10\left({ }^{2} D\right) 4 f^{3} H+9\left({ }^{2} P\right) 4 f^{3} F+7\left({ }^{4} S\right) 5 f^{3} F+7\left({ }^{4} S\right) 4 f^{3} F$ \\
\hline & 206760 & 206576 & 184 & & 1.07 & $30\left({ }^{2} P\right) 4 f^{3} F+30\left({ }^{2} P\right) 4 f^{\prime} G+10\left({ }^{2} D\right) 4 f^{\prime} G+9\left({ }^{2} D\right) 4 f^{3} G+7\left({ }^{2} P\right) 4 f^{3} G+5\left({ }^{2} D\right) 4 f^{3} F$ \\
\hline \multirow[t]{15}{*}{3} & 149062 & 148836 & 225 & 1.57 & 1.61 & $84\left({ }^{4} S\right) 6 p^{5} P+12\left({ }^{2} P\right) 6 p^{3} D$ \\
\hline & 162595 & 162680 & -85 & & 1.16 & $56\left(^{2} D\right) 6 p^{3} F+17\left({ }^{2} D\right) 6 p^{1} F+14\left({ }^{2} P\right) 6 p^{3} D+10\left({ }^{4} S\right) 6 p^{5} P$ \\
\hline & 164439 & 164862 & -424 & & 1.13 & $49\left({ }^{2} D\right) 6 p^{4} F+32\left({ }^{2} D\right) 6 p^{3} D+16\left({ }^{2} D\right) 6 p^{3} F$ \\
\hline & 166374 & 166433 & -59 & & 1.20 & $74\left({ }^{4} S\right) 4 f^{3} F+7\left({ }^{2} P\right) 4 f^{3} F$ \\
\hline & 166699 & 166812 & -113 & & 1.23 & $66\left({ }^{2} D\right) 6 p^{3} D+21\left({ }^{2} D\right) 6 p^{1} F+12\left({ }^{2} D\right) 6 p^{3} F$ \\
\hline & 170250 & 170166 & 85 & & 1.05 & $\begin{array}{l}42\left({ }^{4} S\right) 4 f^{3} F+12\left({ }^{2} D\right) 4 f^{3} F+11\left({ }^{2} P\right) 4 f^{1} F+10\left({ }^{2} D\right) 4 f^{3} G+8\left({ }^{4} S\right) 4 f^{3} F+ \\
5\left({ }^{2} P\right) 4 f^{3} D+5\left({ }^{2} P\right) 4 f^{3} G\end{array}$ \\
\hline & 178306 & 178780 & -474 & & 0.89 & $51\left({ }^{2} D\right) 4 f^{3} G+13\left({ }^{4} S\right) 4 f^{3} F+12\left({ }^{2} P\right) 4 f^{3} G+6\left({ }^{4} S\right) 4 f^{5} F$ \\
\hline & 182377 & 181721 & 656 & & 125 & $42\left({ }^{2} D\right) 4 f^{3} D+12\left({ }^{2} P\right) 4 f^{3} D+12\left({ }^{4} S\right) 4 f^{3} F$ \\
\hline & 184594 & 184505 & 90 & & 1.27 & $57\left({ }^{2} P\right) 6 p^{3} D+9\left({ }^{2} D\right) 6 p^{3} F+8\left({ }^{2} D\right) 4 f^{3} D+7\left({ }^{2} D\right) 6 p^{1} F$ \\
\hline & 186614 & 186511 & 103 & & 1.10 & $67\left({ }^{2} D\right) 4 f^{3} F+15\left({ }^{4} S\right) 4 f^{3} F+7\left({ }^{2} P\right) 4 f^{3} F+5\left({ }^{2} D\right) 4 f^{3} D$ \\
\hline & 189779 & 189591 & 188 & & 1.03 & $55\left({ }^{2} D\right) 4 f^{\prime} F+18\left({ }^{2} P\right) 4 f^{1} F+9\left({ }^{2} D\right) 4 f^{3} D$ \\
\hline & 196156 & 196050 & 106 & & 1.11 & $51\left({ }^{2} P\right) 4 f^{3} F+17\left({ }^{2} P\right) 4 f^{3} D+12\left({ }^{2} P\right) 4 f^{1} F+6\left({ }^{4} S\right) 5 f^{5} F$ \\
\hline & 197586 & 197457 & 129 & & 0.96 & $56\left(\left(^{4} S\right) 5 f^{5} F+21\left({ }^{2} P\right) 4 f^{3} G\right.$ \\
\hline & 197953 & 197931 & 23 & & 1.11 & $29\left({ }^{2} P\right) 4 f^{3} G+25\left({ }^{4} S\right) S f^{5} F+16\left({ }^{2} P\right) 4 f^{1} F+12\left({ }^{2} D\right) 4 f^{1} F+8\left({ }^{2} D\right) 4 f^{3} G$ \\
\hline & 204383 & 204551 & -168 & & 1.22 & $43\left({ }^{2} P\right) 4 f^{3} D+24\left({ }^{2} D\right) 4 f^{3} D+14\left({ }^{2} P\right) 4 f^{1} F+6\left(^{2} P\right) 4 f^{3} F$ \\
\hline \multirow[t]{20}{*}{2} & & -146 & 146 & & 1.44 & $86 p^{4}{ }^{3} P+11 p^{4} D$ \\
\hline & 17099 & 17246 & -148 & & 1.06 & $86 p^{4} D+11 p^{4} P$ \\
\hline & 146962 & 146789 & 173 & 1.70 & 1.67 & $66\left({ }^{4} S\right) 6 p^{3} P+14\left({ }^{4} S\right) 6 p^{3} P+7\left({ }^{2} P\right) 6 p^{3} P+6\left({ }^{2} P\right) 6 p^{3} D$ \\
\hline & 152058 & 151885 & 173 & 1.50 & 1.48 & $64\left({ }^{4} S\right) 6 p^{3} P+19\left({ }^{4} S\right) 6 p^{5} P+8\left(^{2} P\right) 6 p^{1} D$ \\
\hline & 160691 & 160864 & -173 & & 0.95 & $54\left({ }^{2} D\right) 6 p^{3} F+15\left({ }^{2} D\right) 6 p^{3} D+10\left({ }^{4} S\right) 6 p^{3} P+9\left({ }^{2} P\right) 6 p^{3} D$ \\
\hline & 162260 & 162342 & -82 & & 1.18 & $\begin{array}{l}47\left({ }^{2} D\right) 6 p^{3} D+16\left({ }^{2} D\right) 6 p^{3} D+10\left({ }^{2} P\right) 6 p^{3} P+10\left({ }^{2} D\right) 6 p^{3} P+6\left(^{2} P\right) 6 p^{1} D+ \\
6\left({ }^{4} S\right) 6 p^{5} P\end{array}$ \\
\hline & 166880 & 166832 & 48 & & 1.02 & $81\left({ }^{4} S\right) 4 f^{5} F+7\left({ }^{2} P\right) 4 f^{3} D$ \\
\hline & 167066 & 167057 & 9 & & 1.36 & $55\left({ }^{2} D\right) 6 p^{3} P+20\left({ }^{2} D\right) 6 p^{3} D+10\left({ }^{2} D\right) 6 p^{1} D+5\left({ }^{2} P\right) 6 p^{3} P$ \\
\hline & 171990 & 172231 & -242 & & 1.10 & $70\left({ }^{2} D\right) 6 p^{1} D+20\left({ }^{2} D\right) 6 p^{3} P$ \\
\hline & 173734 & 173626 & 108 & & 0.72 & $77\left({ }^{4} S\right) 4 f^{3} F+6\left(\left(^{2} P\right) 4 f^{3} F+5\left({ }^{2} P\right) 4 f^{1} D\right.$ \\
\hline & 177956 & 177530 & 426 & & 1.18 & $59\left({ }^{2} P\right) 6 p^{3} D+19\left({ }^{2} P\right) 6 p^{1} D+14\left({ }^{2} P\right) 6 p^{3} P$ \\
\hline & 181594 & 181005 & 588 & & 0.86 & $\begin{array}{l}41\left({ }^{2} D\right) 4 f^{3} F+15\left({ }^{2} D\right) 4 f^{1} D+14\left({ }^{2} D\right) 4 f^{3} D+7\left({ }^{2} P\right) 4 f^{1} D+7\left({ }^{2} P\right) 4 f^{3} D+ \\
6\left({ }^{4} S\right) 4 f^{5} F\end{array}$ \\
\hline & 184009 & 183888 & 121 & & 1.00 & $44\left({ }^{2} P\right) 6 p^{1} D+17\left({ }^{2} P\right) 6 p^{3} D+14\left({ }^{2} D\right) 6 p^{3} F+8\left({ }^{2} D\right) 4 f^{3} F$ \\
\hline & 186321 & 186108 & 213 & & 1.34 & $27\left({ }^{2} P\right) 6 p^{3} P+27\left({ }^{2} D\right) 4 f^{3} P+11\left({ }^{2} P\right) 6 p^{1} D+7\left({ }^{2} D\right) 6 p^{3} D$ \\
\hline & 186992 & 187118 & -125 & & 1.03 & $31\left({ }^{2} D\right) 4 f^{3} F+25\left({ }^{2} D\right) 4 f^{3} D+12\left({ }^{2} P\right) 6 p^{3} P+10\left({ }^{2} D\right) 4 f^{1} D+6\left({ }^{2} P\right) 4 f^{3} D$ \\
\hline & 188413 & 188681 & -268 & & 1.28 & $38\left({ }^{2} D\right) 4 f^{3} P+25\left({ }^{2} D\right) 4 f^{3} D+12\left({ }^{2} P\right) 6 p^{3} P+8\left(^{2} P\right) 4 f^{3} D$ \\
\hline & 189824 & 190397 & -573 & & 1.10 & $50\left({ }^{2} D\right) 4 f^{\prime} D+21\left({ }^{2} D\right) 4 f^{3} D+7\left({ }^{2} D\right) 4 f^{3} P$ \\
\hline & 197254 & 197145 & 109 & & 0.81 & $43\left({ }^{2} P\right) 4 f^{3} F+22\left({ }^{4} S\right) 5 f^{5} F+17\left({ }^{4} S\right) 5 f^{3} F+6\left({ }^{2} P\right) 4 f^{3} D$ \\
\hline & 197860 & 197695 & 165 & & 0.94 & $68\left({ }^{4} S\right) S f^{5} F+11\left({ }^{2} P\right) 4 f^{3} F+7\left({ }^{4} S\right) S f^{3} F$ \\
\hline & 203360 & 202682 & 678 & & 1.10 & $51\left({ }^{2} P\right) 4 f^{3} D+16\left({ }^{2} D\right) 4 f^{3} P+13\left({ }^{4} S\right) 5 f^{3} F+7\left({ }^{2} D\right) 4 f^{1} D$ \\
\hline \multirow[t]{9}{*}{1} & 9794 & 9739 & 55 & & 1.50 & 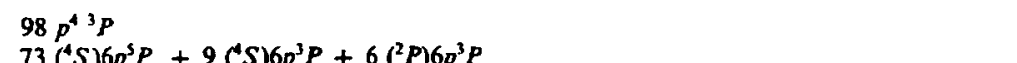 \\
\hline & 146781 & 146609 & 173 & 2.28 & 2.19 & $\begin{array}{l}73\left({ }^{4} S\right) 6 p^{5} P+9\left({ }^{4} S\right) 6 p^{3} P+6\left({ }^{2} P\right) 6 p^{3} P \\
57\left({ }^{4} S\right) 6 p^{3} P+14\left({ }^{4} S\right) 6 p^{5} P+10\left({ }^{2} P\right) 6 p^{1} P+6\left({ }^{2} D\right) 6 p^{3} P\end{array}$ \\
\hline & $\begin{array}{l}150301 \\
158997\end{array}$ & $\begin{array}{l}150097 \\
159349\end{array}$ & $\begin{array}{r}204 \\
-352\end{array}$ & 1.59 & $\begin{array}{l}1.55 \\
1.01\end{array}$ & $\begin{array}{l}57\left({ }^{4} S\right) 6 p^{3} P+14\left({ }^{4} S\right) 6 p^{5} P+10\left({ }^{2} P\right) 6 p^{1} P+6\left(^{2} D\right) 6 p^{3} P \\
38\left({ }^{2} D\right) 6 p^{3} D+26\left({ }^{2} D\right) 6 p^{3} P+12\left({ }^{4} S\right) 6 p^{3} P+5\left({ }^{4} S\right) 6 p^{5} P\end{array}$ \\
\hline & $\begin{array}{l}158997 \\
164512\end{array}$ & $\begin{array}{l}159349 \\
164853\end{array}$ & $\begin{array}{l}-352 \\
-341\end{array}$ & & $\begin{array}{l}1.01 \\
0.97\end{array}$ & $\begin{array}{l}38\left({ }^{2} D\right) 6 p^{3} D+26\left({ }^{2} D\right) 6 p^{1} P+12\left({ }^{4} S\right) 6 p^{3} P+5\left({ }^{4} S\right) 6 p^{5} P \\
41\left({ }^{2} D\right) 6 p^{1} P+32\left({ }^{2} D\right) 6 p^{3} D+8\left({ }^{2} P\right) 6 p^{2} S+6\left({ }^{2} P\right) 6 p^{3} D+6\left({ }^{2} D\right) 6 p^{3} P+5\left({ }^{4} S\right) 6 p^{3} P\end{array}$ \\
\hline & 167174 & 166984 & 190 & & 0.06 & $87\left({ }^{4} S\right) 4 f^{3} F+10\left({ }^{2} P\right) 4 f^{3} D$ \\
\hline & 168086 & 168301 & -215 & & 1.42 & $62\left({ }^{2} D\right) 6 p^{3} P+12\left({ }^{2} D\right) 6 p^{1} P+11\left({ }^{4} S\right) 6 p^{3} P+5\left(^{2} P\right) 6 p^{3} S$ \\
\hline & 175231 & 175094 & 137 & & 0.66 & $66\left({ }^{2} P\right) 6 p^{3} D+20\left({ }^{2} P\right) 6 p^{1} P+6\left({ }^{2} D\right) 6 p^{1} P$ \\
\hline & 178029 & 177703 & 327 & & 1.40 & $59\left(^{2} P\right) 6 p^{3} P+18\left(^{2} P\right) 6 p^{1} P+12\left({ }^{2} P\right) 6 p^{3} S+7\left({ }^{2} P\right) 6 p^{3} D$ \\
\hline & 182134 & 181990 & 144 & & 1.80 & $58\left({ }^{2} P\right) 6 p^{3} S+17\left(^{2} D\right) 6 p^{3} P+11\left({ }^{2} P\right) 6 p^{3} P+7\left(^{2} D\right) 6 p^{1} P$ \\
\hline
\end{tabular}


366 W. Persson, C. -G. Wahlström, G. Bertuccelli, H. O. Di Rocco, J. G. Reyna Almandos and M. Gallardo

Table IV. Continued

\begin{tabular}{|c|c|c|c|c|c|c|}
\hline $\boldsymbol{J}$ & $E$ (obs) & $E$ (calc) & obs - calc & $g_{j}$ (obs) & $g$ (calc) & Percentage composition \\
\hline & 183473 & 183352 & 121 & & 0.91 & $70\left({ }^{2} D\right) 4 f^{1} P+12\left({ }^{2} P\right) 4 f^{3} D$ \\
\hline & 185888 & 186233 & -345 & & 0.99 & $37\left({ }^{2} P\right) 6 p^{1} P+15\left({ }^{2} P\right) 6 p^{3} P+14\left({ }^{2} D\right) 6 p^{3} D+8\left({ }^{2} P\right) 6 p^{3} D$ \\
\hline & 188793 & 188447 & 345 & & 0.54 & $92\left({ }^{2} D\right) 4 f^{3} D$ \\
\hline & 189701 & 190482 & -780 & & 1.37 & $79\left({ }^{2} D\right) 4 f^{3} P+10\left({ }^{2} D\right) 4 f^{1} P+5\left({ }^{2} P\right) 4 f^{3} D$ \\
\hline & 197611 & 197587 & 24 & & 0.05 & $90\left({ }^{4} S\right) S f^{5} F+7\left({ }^{2} P\right) S f^{3} D$ \\
\hline \multirow[t]{8}{*}{$\mathbf{0}$} & 8130 & 8271 & -141 & & & $79 p^{4} p+19 p^{4} S$ \\
\hline & 36103 & 36012 & 91 & & & $78 p^{4} S+19 p^{4} 3 p$ \\
\hline & 152808 & 152577 & 231 & & & $89\left({ }^{4} S\right) 6 p^{3} P+6\left({ }^{2} P\right) 6 p^{3} P$ \\
\hline & 165942 & 166239 & -297 & & & $84\left({ }^{2} D\right) 6 p^{3} P+13\left({ }^{2} P\right) 6 p^{1} S$ \\
\hline & 178055 & 177654 & 400 & & & $83\left({ }^{2} P\right) 6 p^{3} P+13\left({ }^{2} P\right) 6 p^{\prime} S$ \\
\hline & 190491 & 190484 & 7 & & & $67\left({ }^{2} P\right) 6 p^{1} S+15\left(^{2} D\right) 6 p^{3} P+7\left({ }^{4} S\right) 6 p^{3} P+6\left({ }^{2} P\right) 6 p^{3} P+5\left({ }^{2} D\right) 4 f^{3} P$ \\
\hline & & 191529 & & & & $91\left({ }^{2} D\right) 4 f^{3} P$ \\
\hline & 210857 & 210861 & -3 & & & $56 p^{6} ' S+39(' D) 5 d^{\prime} S$ \\
\hline
\end{tabular}

- No levels of $5 s 5 p^{4} 5 d$ bave been established experimentally but the configuration is included in the theoretical treatment of the even configurations (see Section 3.1). 
Table V. Energy parameters (in $\mathrm{cm}^{-1}$ ) for the $5 \mathrm{~s}^{2} 5 \mathrm{p}^{4}+$ $5 s^{2} 5 p^{3}(6 p+4 f+5 f)+5 s^{0} 5 p^{6}+5 s 5 p^{4} 5 d$ configurations of Xe III. Mean error of the least-squares fit $\sigma=I \Sigma\left(E_{\text {obs }}\right.$ $\left.E_{\text {calc }}\right)^{2} /(N-P) J^{1 / 2}=331 \mathrm{~cm}^{-1}$ with $N=73$ known levels and $P=22$ adjustable parameters

\begin{tabular}{|c|c|c|c|}
\hline Parameter & $\operatorname{HF}\left(E_{\mathrm{av}}\right)$ & Fitted & Fitted/HF \\
\hline \multicolumn{4}{|l|}{$5 s^{2} 5 p^{4}$} \\
\hline$E_{\boldsymbol{u r}}$ & & $16905 \pm 160$ & \\
\hline$F^{2}(5 p, 5 p)$ & 50596 & $43681 \pm 1300$ & $0.863 \pm 0.026$ \\
\hline$\zeta_{s p}$ & 6626 & $7995 \pm 300$ & $1.207 \pm 0.045$ \\
\hline \multicolumn{4}{|l|}{$5 s^{2} 5 p^{3} 6 p$} \\
\hline$E_{\mathrm{av}}$ & & $167407 \pm 70$ & \\
\hline$F^{2}(5 p, 5 p)$ & 52840 & $39889 \pm 630$ & $0.755 \pm 0.012$ \\
\hline$F^{2}(5 p, 6 p)$ & 12549 & $11964 \pm 760$ & $0.953 \pm 0.061$ \\
\hline$G^{0}(5 p, 6 p)$ & 2072 & $2007 \pm 80$ & $0.969 \pm 0.039$ \\
\hline$G^{2}(5 p, 6 p)$ & 2982 & $2443 \pm 600$ & $0.819 \pm 0.200$ \\
\hline$\zeta_{s p}$ & 7301 & $8688 \pm 150$ & $1.190 \pm 0.021$ \\
\hline & 939 & $1526 \pm 120$ & $1.625 \pm 0.130$ \\
\hline \multicolumn{4}{|l|}{$5 s^{2} 5 p^{3} 4 f$} \\
\hline$E_{\mathrm{av}}$ & & $187817 \pm 110$ & \\
\hline$F^{2}(5 p, 5 p)$ & 51553 & $38033 \pm 720$ & $0.738 \pm 0.014$ \\
\hline$F^{2}(5 p, 4 f)$ & 29252 & $24040 \pm 900$ & $0.822 \pm 0.031$ \\
\hline$G^{2}(5 p, 4 f)$ & 22845 & $18859 \pm 720$ & $0.825 \pm 0.032$ \\
\hline$G^{4}(5 p, 4 f)$ & 15446 & $9950 \pm 920$ & $0.644 \pm 0.060$ \\
\hline$\zeta_{s, p}$ & 6920 & $8421 \pm 190$ & $1.217 \pm 0.027$ \\
\hline$\zeta_{4}$ & 48 & $0 \pm \quad 60$ & $0.000 \pm 1.250$ \\
\hline \multicolumn{4}{|l|}{$5 s^{2} 5 p^{3} 5 f$} \\
\hline$E_{\mathrm{av}}$ & & $216345 \pm 180$ & \\
\hline$F^{2}(5 p, 5 p)$ & 52207 & $41766(f \mathrm{xx})$ & 0.800 \\
\hline$F^{2}(5 p, 5 f)$ & 12582 & $12582(f x)$ & 1.0 \\
\hline$G^{2}(5 p, 5 f)$ & 8811 & 8811 (fix) & 1.0 \\
\hline$G^{4}(S p, S f)$ & 6274 & 6274 (fix) & 1.0 \\
\hline$\zeta_{\mathrm{sp}}$ & 7102 & 7102 (fix) & 1.0 \\
\hline$\zeta_{s r}$ & 33 & 33 (fix) & 1.0 \\
\hline \multicolumn{4}{|l|}{$p^{6}$} \\
\hline$E_{\mathrm{av}}$ & & $251540 \pm 590$ & \\
\hline \multicolumn{4}{|l|}{$5 s 5 p^{4} S d$} \\
\hline$E_{\mathrm{av}}$ & & 263000 (fix) & \\
\hline$F^{2}(5 p, 5 p)$ & 51463 & 41170 (fix) & 0.800 \\
\hline$F^{2}(S p, S d)$ & 36501 & 36501 (fix) & 1.0 \\
\hline$G^{1}(5 s, 5 p)$ & 68320 & 68320 (fix) & 1.0 \\
\hline$G^{\prime}(5 s, 5 d)$ & 26862 & 26862 (fix) & 1.0 \\
\hline$G^{\prime}(5 p, 5 d)$ & 41701 & 41701 (fix) & 1.0 \\
\hline
\end{tabular}

Table V. Continued

\begin{tabular}{lccl}
\hline Parameter & HF $\left(E_{\text {uv }}\right)$ & Fitted & Fitted/HF \\
\hline$G^{3}(5 p, 5 d)$ & 25951 & $25951($ fix $)$ & 1.0 \\
$\zeta_{s p}$ & 6893 & $6893($ fix $)$ & 1.0 \\
$\zeta_{s}$ & 381 & $381($ fix $)$ & 1.0
\end{tabular}

Configuration

interaction

integrals

$p^{4}-6 p$

\begin{tabular}{cccl}
$R^{2}(5 p 5 p, 5 p 6 p)$ & $5419(.349)^{2}$ & 5419 (fix) & 1.0 \\
$p^{4}-4 f$ & & & \\
$R^{2}(5 p 5 p, 5 p 4 f)$ & $-32918(-.940)$ & $-32918(\mathrm{fix})$ & 1.0 \\
$p^{4}-5 f$ & & & \\
$R^{2}(5 p 5 p, 5 p 5 f)$ & $-20304(-.911)$ & $-20304(\mathrm{fix})$ & 1.0 \\
$p^{4}-5 d$ & & & \\
$R^{1}(555 p, 5 p 5 d)$ & $51463(.998)$ & $51463(\mathrm{fix})$ & 1.0 \\
$R^{2}(5 s 5 p, 5 d 5 p)$ & $37121(.996)$ & $37121(\mathrm{fix})$ & 1.0 \\
$p^{4}-p^{6}$ & & & \\
$R^{1}(5 s 5 s, 5 p 5 p)$ & $67375(1.000)$ & $67375(\mathrm{fix})$ & 1.0 \\
$6 p-4 f$ & & & \\
$R^{2}(5 p 6 p, 5 p 4 f)$ & $3107(.197)$ & $3107(\mathrm{fix})$ & 1.0 \\
$R^{2}(5 p 6 p, 4 / 5 p)$ & $-2428(-.220)$ & $-2428(\mathrm{fix})$ & 1.0 \\
$6 p-5 f$ & & & \\
$R^{2}(5 p 6 p, 5 p 5 f)$ & $-4503(-.530)$ & $-4503(\mathrm{fix})$ & 1.0 \\
$R^{2}(5 p 6 p, 5 / 5 p)$ & $2450(-.360)$ & $2450(\mathrm{fix})$ & 1.0 \\
$6 p-5 d$ & & & \\
$R^{1}(556 p, 5 p 5 d)$ & $7831(.331)$ & $7831(\mathrm{fix})$ & 1.0 \\
$R^{2}(556 p, 5 d 5 p)$ & $-2443(-.199)$ & $-2443(\mathrm{fix})$ & 1.0 \\
$4 f-5 f$ & & & \\
$R^{2}(5 p 4 f, 5 p 5 f)$ & $15068(.859)$ & $8670 \pm 5160$ & $0.575 \pm 0.340$ \\
$R^{2}(5 p 4 f, 5 / 5 p)$ & $13820(.896)$ & $13159 \pm 1584$ & $0.952 \pm 0.120$ \\
$R^{4}(5 p 4 f, 5 / 5 p)$ & $9594(.960)$ & $9594(\mathrm{fix})$ & 1.0 \\
\hline
\end{tabular}

$4 f-5 d$

$R^{\prime}(5 s 4 f, 5 p 5 d) \quad-37462(-.894)-17636(\mathrm{fix}) \quad 0.471$

$R^{2}(5.44,5 d 5 p) \quad-24234(-.941) \quad-24234$ (fix) $\quad 1.0$

Sf-5d

$R^{\prime}(555 f$. Sp5d) $\quad-16823(-.720) \quad-7920$ (fix) $\quad 0.47$

$R^{2}(5 s 5 f, 5 d 5 p)-14589(-.910)-14589$ (fix) $\quad 1.0$ $5 d-p^{6}$

$R^{\prime}(S s S d . S p 5 p) \quad 50988(.998) \quad 50988($ fix $) \quad 1.0$

- The values in parentheses are a measure of the amount of cancellation which occurred in forming the integral. These numbers are the ratio of the true $R^{k}$ value to an $R^{k}$ value calculated using the absolute value of each wavefunction.

Table VI. Comparison between observed and calculated energy-level values (in $\mathrm{cm}^{-1}$ ) and calculated percentage compositions for the $5 s 5 p^{5}+5 s^{2} 5 p^{3}(6 s+7 s+5 d+6 d)$ configurations of Xe III. Eigenvector components larger than $5 \%$ are given. Observed [ 3 ] and calculated $g$, factors are listed

\begin{tabular}{|c|c|c|c|c|c|c|}
\hline $\boldsymbol{J}$ & $E$ (obs) & $E$ (calc) & obs - calc & $g_{\jmath}(\mathrm{obs})$ & $g_{J}$ (calc) & Percentage composition \\
\hline 5 & $\begin{array}{l}132160 \\
200472\end{array}$ & $\begin{array}{l}132443 \\
200436\end{array}$ & $\begin{array}{r}-283 \\
35\end{array}$ & & $\begin{array}{l}1.20 \\
1.20\end{array}$ & $\begin{array}{l}100\left({ }^{2} D\right) 5 d^{3} G \\
100\left({ }^{2} D\right) 6 d^{3} G\end{array}$ \\
\hline 4 & $\begin{array}{l}112272 \\
127782 \\
130174 \\
132712 \\
148536 \\
182716 \\
196538 \\
200051 \\
200426\end{array}$ & $\begin{array}{l}112250 \\
127735 \\
130702 \\
132800 \\
148459 \\
182754 \\
196411 \\
200074 \\
200451\end{array}$ & $\begin{array}{r}21 \\
47 \\
-528 \\
-88 \\
76 \\
-37 \\
127 \\
-24 \\
-25\end{array}$ & & $\begin{array}{l}1.47 \\
1.16 \\
1.20 \\
1.02 \\
1.21 \\
1.46 \\
1.13 \\
1.06 \\
1.21\end{array}$ & $\begin{array}{l}88\left({ }^{4} S\right) S d^{3} D+10\left({ }^{2} P\right) S d^{3} F \\
40\left({ }^{2} D\right) 5 d^{3} G+27\left({ }^{2} D\right) S d^{3} F+16\left({ }^{2} P\right) 5 d^{3} F+9\left({ }^{2} D\right) 5 d^{1} G+7\left({ }^{4} S\right) 5 d^{3} D \\
71\left({ }^{2} D\right) 5 d^{3} F+22\left({ }^{2} D\right) S d^{3} G \\
73\left({ }^{2} D\right) S d^{3} G+25\left({ }^{2} D\right) 5 d^{3} G \\
70\left({ }^{2} P\right) 5 d^{3} F+13\left({ }^{2} D\right) 5 d^{1} G+12\left({ }^{2} D\right) S d^{3} G \\
85\left({ }^{4} S\right) 6 d^{5} D+12\left({ }^{2} P\right) 6 d^{3} F \\
42\left({ }^{2} D\right) 6 d^{3} G+31\left({ }^{2} D\right) 6 d^{3} G+15\left({ }^{2} P\right) 6 d^{3} F+10(4 S) 6 d^{3} D \\
43\left({ }^{2} D\right) 6 d^{4} G+42\left({ }^{2} D\right) 6 d^{1} G+15\left({ }^{2} D\right) 6 d^{3} F \\
82\left({ }^{2} D\right) 6 d^{3} F+16\left({ }^{2} D\right) 6 d^{1} G\end{array}$ \\
\hline 3 & $\begin{array}{l}111605 \\
121230 \\
126120\end{array}$ & $\begin{array}{l}111401 \\
121363 \\
126121\end{array}$ & $\begin{array}{r}204 \\
-133 \\
-1\end{array}$ & & $\begin{array}{l}1.44 \\
1.29 \\
1.12\end{array}$ & $\begin{array}{l}80\left({ }^{4} S\right) 5 d^{5} D+6\left({ }^{2} P\right) 5 d^{3} F+6\left({ }^{2} P\right) S d^{3} D \\
37\left({ }^{4} S\right) S d^{3} D+25\left({ }^{2} D\right) S d^{3} D+15\left({ }^{4} S\right) 5 d^{3} D+6\left({ }^{2} D\right) 5 d^{3} G+6\left({ }^{2} P\right) 5 d^{3} F+5\left({ }^{2} D\right) 5 d^{3} F \\
58\left({ }^{2} D\right) 5 d^{3} F+11\left({ }^{4} S\right) S d^{3} D+11\left({ }^{3} D\right) 5 d^{3} D+10\left({ }^{3} P\right) 5 d^{3} F+8\left({ }^{2} D\right) 5 d^{3} G\end{array}$ \\
\hline
\end{tabular}


Table VI. Continued

\begin{tabular}{|c|c|c|c|c|c|c|}
\hline $\boldsymbol{J}$ & $E$ (obs) & $E$ (calc) & obs - caic & $g_{\jmath}(\mathrm{obs})$ & $g_{j}($ calc $)$ & Percentage composition \\
\hline & 128349 & 128385 & -35 & & 0.87 & $66\left({ }^{2} D\right) 5 d^{3} G+18\left({ }^{2} D\right) 5 d^{3} F+7\left({ }^{4} S\right) 5 d^{5} D$ \\
\hline & 138658 & 139048 & -389 & 1.33 & 1.33 & $83\left({ }^{2} D\right) 6 \sigma^{3} D+8\left({ }^{2} D\right) S d^{3} D$ \\
\hline & 143156 & 143110 & 46 & 1.22 & 1.22 & $38\left({ }^{2} D\right) 5 d^{3} D+21\left({ }^{4} S\right) 5 d^{3} D+12\left({ }^{2} P\right) 5 d^{3} F+10\left({ }^{2} D\right) 5 d^{3} G+7\left({ }^{2} P\right) 5 d^{1} F+6\left({ }^{2} D\right) 6 s^{3} D$ \\
\hline & 145341 & 145259 & 83 & & 1.14 & $56\left({ }^{2} P\right) 5 d^{3} F+11\left({ }^{2} P\right) 5 d^{3} D+7\left({ }^{2} D\right) 6 S^{3} D+6\left({ }^{2} D\right) 5 d^{3} F+6\left({ }^{2} D\right) 5 d^{3} D+5\left({ }^{4} S\right) 5 d^{3} D$ \\
\hline & 148413 & 148691 & -278 & & 1.16 & $42\left({ }^{2} D\right) S d^{3} F+31\left({ }^{2} P\right) 5 d^{3} D+10\left({ }^{4} S\right) 5 d^{3} D$ \\
\hline & 156393 & 156261 & 132 & & 1.15 & $33\left({ }^{2} P\right) 5 d^{3} D+32\left({ }^{2} P\right) 5 d^{4} F+15\left({ }^{2} D\right) 5 d^{1} F+6\left({ }^{2} D\right) 5 d^{3} F+5\left({ }^{2} D\right) 5 d^{3} D$ \\
\hline & 162958 & 163174 & -216 & & 1.04 & $40\left({ }^{2} P\right) 5 d^{\prime} F+35\left({ }^{2} D\right) 5 d^{\prime} F+7\left({ }^{2} P\right) 5 d D+5\left({ }^{4} S\right) 5 d^{3} D+5\left({ }^{2} D\right) 6 d^{\prime} F$ \\
\hline & 182464 & 182430 & 34 & & 1.44 & $79\left({ }^{4} S\right) 6 d^{5} D+6\left(\left(^{2} P\right) 6 d^{3} D+6\left({ }^{2} P\right) 6 d^{3} F\right.$ \\
\hline & 186384 & 186311 & 74 & & 1.28 & $71\left({ }^{4} S\right) 6 d^{3} D+9\left({ }^{2} P\right) 6 d^{1} F+6\left({ }^{4} S\right) 6 d^{3} D$ \\
\hline & 196262 & 196304 & -42 & & 0.93 & $58\left({ }^{2} D\right) 6 d^{3} G+11\left({ }^{4} S\right) 6 d^{3} D+11\left({ }^{2} D\right) 6 d^{3} F+8\left({ }^{2} P\right) 6 d^{1} F+7\left({ }^{2} D\right) 6 d^{3} D$ \\
\hline & 196609 & 196640 & -31 & & 1.13 & $\begin{array}{l}43\left({ }^{2} D\right) 6 d^{3} F+13\left({ }^{2} D\right) 6 d^{3} G+11\left({ }^{2} D\right) 6 d^{3} D+9\left({ }^{2} P\right) 6 d^{3} D+9\left({ }^{4} S\right) 6 d^{3} D+ \\
6\left({ }^{2} D\right) 6 d^{1} F+6\left({ }^{2} P\right) 6 d^{3} F\end{array}$ \\
\hline & 200033 & 200054 & -21 & & 1.33 & $99\left({ }^{2} D\right) 7 s^{3} D$ \\
\hline & 200650 & 200815 & -165 & & 1.25 & $64\left({ }^{2} D\right) 6 d^{3} D+30\left({ }^{2} D\right) 6 d^{3} F$ \\
\hline & 203845 & 203921 & -76 & & 1.04 & $71\left({ }^{2} D\right) 6 d^{1} F+11\left({ }^{2} D\right) 6 d^{3} D+5\left({ }^{2} D\right) 6 d^{3} G$ \\
\hline \multirow[t]{23}{*}{2} & $\begin{array}{r}98262 \\
111856\end{array}$ & $\begin{array}{r}98217 \\
111593\end{array}$ & $\begin{array}{r}45 \\
264\end{array}$ & & 1.50 & \\
\hline & $\begin{array}{l}111856 \\
117240\end{array}$ & $\begin{array}{l}111593 \\
117130\end{array}$ & $\begin{array}{l}264 \\
110\end{array}$ & & 1.40 & $\begin{array}{l}78\left({ }^{4} S\right) 5 d^{5} D+6\left({ }^{2} P\right) 5 d^{3} D \\
27\left({ }^{4} S\right) 5 d^{3} D+17\left({ }^{2} D\right) 5 d^{3} D+16\left({ }^{2} D\right) 5 d^{3} F+15\left({ }^{4} S\right) S d^{5} D+12\left({ }^{2} P\right) 5 d^{4} D+6\left({ }^{2} P\right) 5 d^{3} F\end{array}$ \\
\hline & 121476 & 121343 & 133 & 1.95 & $\begin{array}{l}1.09 \\
1.93\end{array}$ & $\begin{array}{l}27(S) 5 d^{3} D+17\left({ }^{2} D\right) 5 d^{3} D+16\left({ }^{2} D\right) 5 d^{3} F+15\left({ }^{4} S\right) S d^{5} D+12\left({ }^{2} P\right) 5 d^{4} D+6\left({ }^{2} P\right) 5 d^{3} F \\
87\left({ }^{4} S\right) 6 s^{5} S+10\left({ }^{2} P\right) 6 s^{3} P\end{array}$ \\
\hline & 124691 & 124721 & -30 & & 0.86 & $52\left({ }^{2} D\right) 5 d^{3} F+18\left({ }^{2} D\right) 5 d^{3} D+16\left({ }^{4} S\right) 5 d^{3} D+11\left({ }^{2} P\right) 5 d^{3} F$ \\
\hline & 134667 & 134767 & -99 & 1.18 & 1.20 & $33\left({ }^{2} D\right) 6 s^{3} D+20\left({ }^{2} D\right) 6 s^{1} D+12\left({ }^{2} P\right) S d^{1} D+11\left({ }^{2} P\right) 6 s^{3} P+6\left({ }^{4} S\right) 6 s^{3} S+6\left({ }^{2} D\right) 5 d^{1} D$ \\
\hline & 136367 & 136137 & 231 & 0.90 & 1.08 & $22\left({ }^{2} D\right) 6 s^{3} D+16\left(^{2} D\right) 5 d^{3} D+15\left(^{2} P\right) 5 d^{1} D+15\left(^{2} D\right) 5 d^{1} D+10\left(^{2} P\right) 5 d^{3} F+8\left({ }^{2} P\right) 5 d^{3} D$ \\
\hline & 142064 & 141824 & 241 & 1.12 & 1.17 & $24\left({ }^{2} D\right) 5 d^{3} D+22\left({ }^{4} S\right) S d^{3} D+18\left({ }^{2} P\right) 5 d^{3} D+11\left({ }^{2} D\right) 6 S^{3} D+10\left({ }^{2} P\right) 5 d^{1} D+6\left(^{2} P\right) 6 s^{3} P$ \\
\hline & 143048 & 143109 & -61 & 0.96 & 1.02 & $54\left({ }^{2} D\right) 6 s^{\prime} D+19\left({ }^{2} D\right) 6 s^{3} D+12\left({ }^{2} P\right) 5 d^{3} F+5\left({ }^{2} P\right) 5 d^{\prime} D$ \\
\hline & 145300 & 145112 & 188 & 0.81 & 0.85 & $50\left({ }^{2} P\right) 5 d^{3} F+18\left({ }^{2} D\right) 5 d^{3} F+13\left({ }^{2} P\right) 5 d^{3} P+8\left({ }^{2} D\right) 5 d^{3} D$ \\
\hline & 148370 & 148590 & -220 & & 1.37 & $39\left({ }^{2} D\right) 5 d^{3} P+14 p^{5} P+10\left({ }^{3} P\right) 5 d^{3} P+9\left({ }^{2} D\right) 5 d^{3} D+6\left({ }^{2} P\right) 5 d^{1} D+6\left({ }^{2} P\right) 5 d^{3} D$ \\
\hline & 150404 & 150246 & 159 & & 1.37 & $54\left({ }^{2} P\right) 5 d^{3} P+12\left({ }^{2} D\right) 5 d^{3} P+6\left({ }^{2} D\right) 5 d^{1} D+5\left({ }^{2} P\right) 6 s^{3} P$ \\
\hline & 153893 & 153614 & 279 & & 1.22 & $\begin{array}{l}28\left({ }^{2} P\right) 5 d^{3} D+12\left({ }^{2} D\right) 5 d^{1} D+11\left({ }^{2} D\right) 5 d^{3} P+11\left({ }^{2} P\right) 6 \sigma^{3} P+10\left({ }^{4} S\right) 5 d^{3} D+ \\
6\left({ }^{2} P\right) 5 d^{1} D+6\left({ }^{2} D\right) 5 d^{3} D\end{array}$ \\
\hline & 158928 & 159117 & -189 & & 1.41 & $53\left({ }^{2} P\right) 6 s^{3} P+18\left({ }^{2} D\right) 5 d^{3} P+8\left({ }^{2} D\right) 6 s^{3} D+8\left({ }^{2} D\right) 6 s^{1} D$ \\
\hline & 161810 & 162283 & -473 & & 1.04 & $39\left({ }^{2} D\right) 5 d^{1} D+20\left({ }^{2} P\right) 5 d^{3} D+19\left({ }^{2} P\right) 5 d^{3} D+7\left({ }^{4} S\right) 5 d^{3} D$ \\
\hline & 182338 & 182357 & -19 & & 1.66 & $42\left({ }^{4} S\right) 6 d^{3} D+41\left({ }^{4} S\right) 7 s^{3} S+5\left({ }^{2} P\right) 7 s^{3} P$ \\
\hline & 182483 & 182450 & 33 & & 1.69 & $45\left({ }^{4} S\right) 7 s^{3} S+38\left({ }^{4} S\right) 6 d^{5} D+6\left({ }^{2} P\right) 7 s^{3} P$ \\
\hline & 185121 & 185220 & -99 & & 1.16 & $62\left({ }^{4} S\right) 6 d^{3} D+10\left({ }^{2} P\right) 6 d^{1} D+6\left({ }^{2} D\right) 6 d^{3} D+6\left({ }^{4} S\right) 6 d^{3} D$ \\
\hline & 195978 & 195970 & 7 & & 0.95 & $47\left({ }^{2} D\right) 6 d^{3} F+11\left({ }^{2} D\right) 7 s^{3} D+7\left({ }^{4} S\right) 6 d^{3} D+7\left({ }^{2} D\right) 6 d d^{1} D$ \\
\hline & 196141 & 196103 & 38 & & 1.16 & $38\left({ }^{2} D\right) 7 s^{3} D+20\left({ }^{2} D\right) 7 s^{1} D+13\left({ }^{2} D\right) 6 d^{3} F+13\left({ }^{2} P\right) 7 s^{3} P+7\left({ }^{4} S\right) 7 s^{3} S$ \\
\hline & 198492 & 198506 & -14 & & 1.26 & $37\left({ }^{2} D\right) 6 d^{3} D+22\left({ }^{2} D\right) 6 d^{3} P+10\left({ }^{4} S\right) 6 d^{3} D+9\left({ }^{2} P\right) 6 d^{3} P$ \\
\hline & 200540 & $200 \$ 20$ & 20 & & 1.06 & $64\left({ }^{2} D\right) 7 s^{1} D+34\left({ }^{2} D\right) 7 s^{3} D$ \\
\hline & 201512 & 201507 & 5 & & 1.22 & $44\left({ }^{2} D\right) 6 d^{3} D+30\left({ }^{2} D\right) 6 d^{3} P+13\left({ }^{2} D\right) 6 d^{1} D+6\left({ }^{2} D\right) 6 d^{3} F$ \\
\hline & 203376 & 203194 & 183 & & 1.13 & $54\left({ }^{2} D\right) 6 d^{\prime} D+30\left({ }^{2} D\right) 6 d^{3} P+7\left({ }^{2} D\right) 6 d^{3} F$ \\
\hline \multirow[t]{23}{*}{1} & 103568 & 103639 & -71 & & 1.45 & $62 p^{5}{ }^{3} P+14\left({ }^{2} D\right) 5 d^{3} P+8\left({ }^{2} P\right) 5 d^{3} P$ \\
\hline & 112450 & 112328 & 122 & & 1.48 & $87(S) 5 d^{3} D+6 p^{3}{ }^{3} P$ \\
\hline & 119026 & 118935 & 91 & & 1.01 & $44\left({ }^{2} D\right) 5 d^{1} P+28 p^{5} P+7\left({ }^{2} P\right) 5 d^{4} P+7 p^{5}{ }^{3} P+6\left({ }^{4} S\right) 5 d^{3} D$ \\
\hline & 121923 & 122282 & -359 & & 0.60 & $51\left({ }^{4} S\right) 5 d^{3} D+31\left({ }^{2} D\right) 5 d^{3} D+5\left({ }^{2} P\right) 5 d^{3} D$ \\
\hline & 125617 & 125594 & 23 & 1.77 & 1.80 & $78\left({ }^{4} S\right) 63^{3} S+7\left({ }^{2} P\right) 6 s^{\prime} P$ \\
\hline & 133234 & 133412 & -178 & 0.38 & 0.65 & $36\left({ }^{2} D\right) 5 d^{3} D+25\left({ }^{2} D\right) 6 s^{3} D+22\left({ }^{2} P\right) 5 d^{3} D+6\left({ }^{4} S\right) 6 s^{3} S$ \\
\hline & 138145 & 138128 & 17 & 0.50 & 0.70 & $49\left({ }^{2} D\right) 6 s^{3} D+17\left({ }^{2} D\right) S d^{3} D+9\left({ }^{2} P\right) 5 d^{3} D+6\left(^{2} P\right) 6 s^{1} P+6\left({ }^{4} S\right) 6 s^{3} S+5\left({ }^{4} S\right) S d^{3} D$ \\
\hline & 140731 & 140464 & 267 & & 1.53 & $44\left({ }^{2} P\right) 5 d^{3} P+19\left({ }^{2} D\right) 5 d^{3} S+18\left({ }^{2} D\right) 5 d^{3} P$ \\
\hline & 147797 & 147630 & 168 & & 1.70 & $49\left({ }^{2} D\right) 5 d^{3} S+20\left({ }^{2} P\right) 63^{3} P+18\left({ }^{2} D\right) 5 d^{3} P$ \\
\hline & 151482 & 151584 & -102 & 1.47 & 1.46 & $46\left({ }^{2} P\right) 63^{3} P+22\left({ }^{2} P\right) 6 s^{1} P+15\left({ }^{2} D\right) 5 d^{3} S+12\left({ }^{2} P\right) 5 d^{3} P$ \\
\hline & 154639 & 154546 & 93 & & 1.30 & $\begin{array}{l}20\left({ }^{2} D\right) S d^{3} P+18 p^{5} P+15\left({ }^{2} P\right) 5 d^{3} P+13\left({ }^{2} P\right) 6 s^{1} P+9\left({ }^{2} D\right) 5 d^{3} S+ \\
7 p^{5}{ }^{3} P+7\left({ }^{2} D\right) 5 d^{1} P\end{array}$ \\
\hline & 155401 & 155333 & 68 & & 0.64 & $45\left({ }^{2} P\right) S d^{3} D+16\left({ }^{4} S\right) 5 d^{3} D+12\left({ }^{2} P\right) 5 d^{1} P+8\left({ }^{2} D\right) 5 d^{3} D+6\left({ }^{4} S\right) 6 d^{3} D$ \\
\hline & 159388 & 159126 & 262 & & 1.20 & $32\left({ }^{2} P\right) 6 s^{1} P+20\left({ }^{2} P\right) 6 s^{3} P+14\left({ }^{2} D\right) 5 d^{3} P+13\left({ }^{2} D\right) 6 s^{3} D+6 p^{5}{ }^{3} P+6\left({ }^{2} P\right) 5 d^{3} P$ \\
\hline & 163527 & 163844 & -316 & & 0.99 & $33\left({ }^{2} D\right) 5 d^{1} P+27 p^{5} P+10\left({ }^{2} P\right) 6 s^{4} P+7\left({ }^{2} P\right) 5 d^{3} D+7\left({ }^{2} P\right) 5 d^{3} P$ \\
\hline & 175052 & 174482 & 570 & & 0.99 & $64\left({ }^{2} P\right) 5 d^{\prime} P+8 p^{3} P+6\left({ }^{2} P\right) 6 d^{1} P+5\left({ }^{4} S\right) 5 d^{3} D$ \\
\hline & 182551 & 182496 & 55 & & 1.45 & $85\left({ }^{4} S\right) 6 d^{3} D+8\left({ }^{2} P\right) 6 d^{3} P$ \\
\hline & 183786 & 183795 & -9 & & 1.84 & $84\left({ }^{4} S\right) 7 s^{3} S+8\left({ }^{2} P\right) 7 s^{1} P$ \\
\hline & 186589 & 186645 & -56 & & 0.60 & $76\left({ }^{4} S\right) 6 d^{3} D+5\left({ }^{2} P\right) 6 d^{3} D+5\left({ }^{2} P\right) 6 d^{1} P$ \\
\hline & 195907 & 195948 & -41 & & 0.77 & $57\left({ }^{2} D\right) 7 s^{3} D+11\left({ }^{2} D\right) 6 d^{3} D+8\left({ }^{4} s\right) 7 s^{3} S+7\left({ }^{2} P\right) 7 s^{1} P$ \\
\hline & 196877 & 196881 & -5 & & 0.74 & $45\left({ }^{2} D\right) 6 d^{3} D+18\left({ }^{2} D\right) 75^{3} D+12\left({ }^{2} D\right) 6 d^{1} P$ \\
\hline & 199104 & 199122 & -18 & & 1.58 & $45\left({ }^{2} D\right) 6 d^{3} S+21\left({ }^{2} D\right) 6 d^{3} P+10\left({ }^{2} P\right) 6 d^{3} P+7\left({ }^{2} D\right) 6 d^{1} P$ \\
\hline & 202036 & 202066 & -31 & & 1.16 & $46\left({ }^{2} D\right) 6 d^{3} P+22\left({ }^{2} D\right) 6 d^{1} P+22\left({ }^{2} D\right) 6 d^{3} D$ \\
\hline & 202806 & 202589 & 217 & & 1.45 & $38\left({ }^{2} D\right) 6 d^{3} S+37\left({ }^{2} D\right) 6 d^{1} P+16\left({ }^{2} D\right) 6 d^{3} P$ \\
\hline \multirow[t]{9}{*}{0} & 108334 & 108380 & -46 & & & $55 p^{5}{ }^{3} P+22\left({ }^{4} S\right) 5 d^{3} D+12\left({ }^{2} D\right) 5 d^{3} P+10\left({ }^{2} P\right) 5 d^{3} P$ \\
\hline & 112694 & 112591 & 103 & & & $69\left({ }^{4} S\right) 5 d^{5} D+20 p^{5} P+9\left(^{2} D\right) 5 d^{3} P$ \\
\hline & & 126871 & & & & $85\left({ }^{2} D\right) 5 d^{\prime} S+8\left({ }^{2} P\right) 5 d^{3} P$ \\
\hline & 140438 & 140556 & -118 & & & $42\left({ }^{2} P\right) 5 d^{3} P+31\left({ }^{2} D\right) 5 d^{3} P+12\left({ }^{2} D\right) 5 d^{1} S+8\left({ }^{2} P\right) 6 s^{3} P$ \\
\hline & 150505 & 150324 & 181 & & & $87\left({ }^{2} P\right) 6 s^{3} P+10\left({ }^{2} P\right) 5 d^{3} P$ \\
\hline & 160734 & 160743 & -9 & & & $43\left({ }^{2} D\right) 5 d^{3} P+28\left(^{2} P\right) 5 d^{3} P+20 p^{3}{ }^{3} P$ \\
\hline & 182522 & 182482 & 40 & & & $86\left({ }^{4} S\right) 6 d^{3} D+11\left(f^{2} P\right) 6 d^{3} P$ \\
\hline & 197091 & 197024 & 67 & & & $58\left({ }^{2} D\right) 6 d^{\prime} S+19\left(^{2} D\right) 6 d^{3} P+15\left({ }^{2} P\right) 6 d^{3} P+8\left({ }^{4} S\right) 6 d^{5} D$ \\
\hline & 201618 & 201847 & -228 & & & $62\left({ }^{2} D\right) 6 d^{2} 3+32\left({ }^{2} D\right) 6 d^{2} S$ \\
\hline
\end{tabular}


Table VII. Energy parameters (in $\mathrm{cm}^{-1}$ ) for the $5 s 5 p^{3}+5 s^{2} 5 p^{3}(5 d+6 d+6 s+7 s)$ configurations of $X e$ III. Mean error of the least-squares fit $\sigma=I \Sigma\left(E_{o b s}-\right.$ $\left.E_{\text {ralk }}\right)^{2} /(N-P) J^{1 / 2}=217 \mathrm{~cm}^{-1}$ with $N=83$ known levels and $P=30$ adjustable parameters

\begin{tabular}{|c|c|c|c|}
\hline Parameter & $H F\left(E_{\text {av }}\right)$ & Fitted & Fitted/HF \\
\hline \multicolumn{4}{|l|}{$p^{3}$} \\
\hline$E_{\mathrm{av}}$ & & $123209 \pm 430$ & \\
\hline$G^{\prime}(S s, 5 p)$ & 67295 & $47314 \pm 950$ & $0.703 \pm 0.014$ \\
\hline$\zeta_{s p_{p}}$ & 6605 & $7760 \pm 310$ & $1.175 \pm 0.047$ \\
\hline \multicolumn{4}{|l|}{ Gs } \\
\hline$E_{\mathrm{av}}$ & & $140786 \pm 90$ & \\
\hline$F^{2}(5 p, 5 p)$ & 52231 & $41089 \pm 630$ & $0.787 \pm 0.012$ \\
\hline$G^{\prime}(5 p, 6 s)$ & 4544 & $3795 \pm 250$ & $0.835 \pm 0.055$ \\
\hline$\zeta_{s}$ & 7169 & $8078 \pm 190$ & $1.127 \pm 0.027$ \\
\hline \multicolumn{4}{|l|}{$7 s$} \\
\hline$E_{\mathrm{av}}$ & & $200200 \pm 160$ & \\
\hline$F^{2}(5 p, 5 p)$ & 52644 & $40033 \pm 1320$ & $0.760 \pm 0.025$ \\
\hline$G^{\prime}(5 p, 7 s)$ & 1376 & $1072 \pm 300$ & $0.779 \pm 0.220$ \\
\hline$\zeta_{s p}$ & 7261 & $8072 \pm 490$ & $1.112 \pm 0.360$ \\
\hline \multirow{2}{*}{\multicolumn{4}{|c|}{$136928 \pm 130$}} \\
\hline & & & \\
\hline$F^{2}(5 p .5 p)$ & 51522 & $39438 \pm 440$ & $0.765 \pm 0.009$ \\
\hline$F^{2}(5 p, S d)$ & 35641 & $29232 \pm 440$ & $0.820 \pm 0.012$ \\
\hline$G^{\prime}(5 p, 5 d)$ & 40265 & $28868 \pm 570$ & $0.717 \pm 0.014$ \\
\hline$G^{3}(5 p, 5 d)$ & 25050 & $16810 \pm$ & $0.671 \pm 0.022$ \\
\hline$\zeta_{s p}$ & 6933 & $8308 \pm 100$ & $1.198 \pm 0.014$ \\
\hline$\zeta_{s s}$ & 358 & $467 \pm$ & $1.304 \pm 0.170$ \\
\hline \multicolumn{4}{|l|}{$6 d$} \\
\hline$E_{\alpha v}$ & & $\begin{array}{r}200589 \pm 140 \\
40006 \pm 630\end{array}$ & \\
\hline $\begin{array}{l}F^{2}(S p, S p) \\
F^{2}(S p, 6 d)\end{array}$ & $\begin{array}{r}52621 \\
8853\end{array}$ & $6757 \pm 820$ & $0.760 \pm 0.012$ \\
\hline$G^{\prime}(5 p, 6 d)$ & 6482 & $2384 \pm 580$ & $0.368 \pm 0.089$ \\
\hline$G^{3}(5 p, 6 d)$ & 4400 & $1089 \pm 710$ & $0.248 \pm 0.160$ \\
\hline$\zeta_{S_{p}}$ & 7239 & $8456 \pm 240$ & $1.168 \pm 0.033$ \\
\hline$\zeta$ & 98 & $161 \pm 52$ & $1.643 \pm 0.530$ \\
\hline \multicolumn{4}{|l|}{$\begin{array}{l}\text { Configuration } \\
\text { interaction } \\
\text { integrals }\end{array}$} \\
\hline \multicolumn{4}{|l|}{$p^{3}-6 . s$} \\
\hline \multirow{2}{*}{\multicolumn{4}{|c|}{$p^{3}-7 s$}} \\
\hline & & & \\
\hline$R^{\prime}(5 p 5 p, 5 s 7 s)$ & $-318(-.021)$ & $-318(f i x)$ & 1.0 \\
\hline \multicolumn{4}{|l|}{$p^{3}-5 d$} \\
\hline \multirow{2}{*}{\multicolumn{4}{|c|}{$p^{5}-6 d$}} \\
\hline & & & \\
\hline$R^{\prime}(5 p 5 p, 5 s 6 d)$ & $20212(.919)$ & $16016 \pm 1760$ & $0.792 \pm 0.087$ \\
\hline \multicolumn{4}{|l|}{$6 s-7 s$} \\
\hline$R^{\prime}(5 p 6 s, 7 s 5 p)$ & $2426(.370)$ & $2426(f x)$ & 1.0 \\
\hline \multicolumn{4}{|l|}{$6 s-5 d$} \\
\hline$R^{2}(5 p 6 s, 5 p 5 d)$ & $-9991(-.507)$ & -9991 (fix) & 1.0 \\
\hline$R^{\prime}(5 p 6 s, 5 d 5 p)$ & $-2966(-.141)$ & -2966 (fix) & 1.0 \\
\hline \multicolumn{4}{|l|}{$6 s-6 d$} \\
\hline$R^{2}(S p 6 s, S p 6 d)$ & $3214(.379)$ & 3214 (fix) & 1.0 \\
\hline$R^{\prime}(5 p 6 s, 6 d 5 p)$ & $271(.031)$ & $271(f i x)$ & 1.0 \\
\hline \multicolumn{4}{|l|}{$7 s-5 d$} \\
\hline$R^{2}(5 p 7 s, 5 p 5 d)$ & $-4994(-.453)$ & $-4994(f x)$ & 1.0 \\
\hline$R^{\prime}(5 p 7 s, 5 d 5 p)$ & $-2042(-.167)$ & $-2042(f x)$ & 1.0 \\
\hline \multicolumn{4}{|l|}{$7 s-6 d$} \\
\hline$R^{2}(5 p 7 s, 5 p 6 d)$ & $-2138(-.384)$ & -2138 (fix) & 1.0 \\
\hline$R^{\prime}(5 p 7 s, 6 d 5 p)$ & $-148(-.029)$ & $-148(f x)$ & 1.0 \\
\hline \multicolumn{4}{|l|}{$5 d-6 d$} \\
\hline$R^{2}(5 p 5 d, 5 p 6 d)$ & $11112(.756)$ & $11638 \pm 1720$ & $1.047 \pm 0.160$ \\
\hline$R^{1}(S p S d, 6 d S p)$ & $15402(.901)$ & $14647 \pm 1150$ & $0.951 \pm 0.075$ \\
\hline$R^{3}(5 p 5 d, 6 d 5 p)$ & $9957(.943)$ & 5000 (fix) & 0.502 \\
\hline
\end{tabular}

- The values in parentheses are a measure of the amount of cancellation which occurred in forming the integral. These numbers are the ratio of the true $\boldsymbol{R}^{k}$ value to an $\boldsymbol{R}^{k}$ value calculated using the absolute value of each wavefunction.
Table VIII. Comparison between xenon $N_{4,5} O O$ Auger spectrum and optical spectrum of $X e^{2+}$. Optical data: Present work. Auger data: L. O. Werme, T. Bergmark and K. Siegbahn [22J

\begin{tabular}{|c|c|c|c|c|c|c|}
\hline \multicolumn{3}{|c|}{ Auger data } & \multicolumn{3}{|l|}{ Optical data } & \multirow{2}{*}{$\begin{array}{l}\text { Energy } \\
\text { difference } \\
\text { Optical-Auger } \\
\text { ev }\end{array}$} \\
\hline Line No. & Intensity & $\begin{array}{l}\text { Energy } \\
\text { eV }\end{array}$ & Classification & $\begin{array}{l}\% 5 \\
1 P\end{array}$ & $\begin{array}{l}5 p^{5} \\
{ }^{3} P\end{array}$ & \\
\hline 1,3 & 15,86 & 0 & $5 s^{2} 5 p^{4} P_{2}$ & & & 0.00 \\
\hline 2. 6 & 43,39 & 1.21 & & & & 0.00 \\
\hline,- 5 &,- 48 & 1.00 & ${ }^{3} P_{0}$ & & & +0.01 \\
\hline 4. 7 & 104,97 & 2.12 & $' D_{2}$ & & & 0.00 \\
\hline 8,9 & 100,73 & 4.49 & ${ }^{\prime} S_{0}$ & & & 0.00 \\
\hline 13,15 & 11,7 & 12.17 & $5 s 5 p^{5}{ }^{3} P_{2}$ & - & 75 & +0.01 \\
\hline $14,-$ & $10,-$ & 12.85 & ${ }^{3} P_{1}^{2}$ & 5 & 62 & -0.01 \\
\hline 16,18 & 62,80 & 14.76 & $\left.{ }^{2} D\right) 5 d{ }^{\prime} P_{1}$ & 28 & 7 & 0.00 \\
\hline 17.19 & 3. 8 & 15.13 & $\left({ }^{4} S\right) S d{ }^{3} D_{1}$ & 2 & 1 & -0.01 \\
\hline 20,23 & 7,11 & 17.44 & $\left({ }^{2} P\right) S d{ }^{3} P_{1}$ & 2 & - & +0.01 \\
\hline 22,25 & 41,74 & 19.17 & $\left.{ }^{2} D\right) 5 d{ }^{3} P_{1}$ & 18 & 7 & 0.00 \\
\hline,$- 26^{b}$ &,- 31 & 19.73 & $\left.{ }^{2} P\right) 6 s{ }^{1} P_{t}$ & 1 & 6 & +0.03 \\
\hline 24,27 & 90,134 & 20.27 & $5 s s p^{5}{ }^{1} P_{1}$ & 27 & 2 & 0.00 \\
\hline 26.28 & 31, 25 & 21.70 & $\left.{ }^{2} P\right) S d{ }^{\prime} P_{1}$ & 8 & $\mathbf{1}$ & 0.00 \\
\hline 29,30 & 107,133 & 26.13 & $5 s^{0} 5 p^{6}{ }^{1} S_{0}$ & & & +0.01 \\
\hline
\end{tabular}

- $1 \mathrm{eV}=8065.545 \mathrm{~cm}^{-1}$.

b Doubly classified.

Table IX. Classification of $\mathrm{Xe}^{2+}$ laser lines. Data on laser lines are from Beck et al. [9]

\begin{tabular}{|c|c|c|c|}
\hline \multicolumn{2}{|l|}{ Laser data } & \multicolumn{2}{|c|}{ Spontaneous data } \\
\hline Wavelength & Comment" & Intensity & Classification \\
\hline $\begin{array}{l}2477.18 \\
2691.939\end{array}$ & \multirow[t]{2}{*}{$\mathrm{Xe}^{2+} ?$} & 0 & $\begin{array}{l}\left({ }^{S} S\right) S d{ }^{5} D_{1}-\left({ }^{4} S\right) 6 p{ }^{3} P_{0} \\
\mathrm{Xe} \Gamma V^{b}\end{array}$ \\
\hline 3079.738 & & & \\
\hline 3305.99 & \multirow{22}{*}{$\begin{array}{l}\mathrm{Xe}^{2+} ? \\
\mathrm{Xe}^{3+} ?\end{array}$} & & $\mathrm{Xe} J \mathrm{~V}^{\mathrm{e}}$ \\
\hline 3349.74 & & 12 & $\left({ }^{2} D\right) 6 s^{3} D_{2}-\left({ }^{2} D\right) 6 p{ }^{1} P_{1}$ \\
\hline 3454.248 & & 16 & $\left(^{2} D\right) 6 s s^{1} D_{2}-\left({ }^{2} D\right) 6 p \quad{ }^{2} D_{2}$ \\
\hline 3596.61 & & 17 & $\left({ }^{2} D\right) 6 s^{3} D_{1}-\left({ }^{2} D\right) 6 p{ }^{3} P_{0}$ \\
\hline 3669.21 & & & $\mathrm{Xe} I \mathrm{~V}^{\phi}$ \\
\hline 3745.71 & & 18 & $\left({ }^{2} P\right) 5 d d^{3} F_{2}-\left({ }^{2} D\right) 6 p \quad{ }^{1} D_{2}$ \\
\hline 3780.990 & & 28 & $\left({ }^{4} S\right) 6 s S^{3} S_{1}-\left({ }^{4} S\right) 6 p{ }^{3} P_{2}$ \\
\hline 4050.05 & & 22 & $\left({ }^{4} S\right) 6{ }^{3} S_{1}-\left({ }^{4} S\right) 6 p{ }^{3} P_{1}$ \\
\hline 4060.48 & & 25 & $\left({ }^{2} P\right) 6 s \quad{ }^{1} P_{1}-\left({ }^{2} P\right) 6 p \quad{ }^{2} D_{2}$ \\
\hline 4214.01 & & 24 & $\left({ }^{2} D\right) S d^{3} D_{3}-\left({ }^{4} S\right) 4 f^{5} F_{2}$ \\
\hline 4240.24 & & 16 & $\left({ }^{2} D\right) 5 d d^{1} F_{3}-\left({ }^{2} D\right) 6 p \quad{ }^{1} D_{2}$ \\
\hline 4272.59 & & 17 & $\left({ }^{2} D\right) 5 d^{3} D_{3}-\left({ }^{2} D\right) 6 p{ }^{3} F_{4}$ \\
\hline 4285.88 & & 20 & $\left.\left({ }^{2} D\right) 6 s S^{1} D_{2}-f^{4} S\right) 4 f^{5} F_{3}$ \\
\hline 4413.14 & & 8 & $\left({ }^{2} P\right) 5 d{ }^{3} D_{1}-\left({ }^{2} P\right) 6 P{ }^{3} P_{0}$ \\
\hline 4434.15 & & 15 & $\left.{ }^{2} D\right) 6 s{ }^{3} D_{1}-\left({ }^{2} D\right) 6 p{ }^{3} F_{2}$ \\
\hline 4673.68 & & $18 \mathrm{a}$ & $\left(^{2} D\right) 6 s{ }^{1} D_{2}-\left({ }^{2} D\right) 6 p \quad{ }^{\prime} F_{3}$ \\
\hline 4683.54 & & 20 & $\left({ }^{4} S\right) 6 s{ }^{3} S_{1}-\left({ }^{4} S\right) 6 p{ }^{3} P_{2}$ \\
\hline 4723.57 & & 16 & $\left({ }^{4} S\right) 6 s{ }^{3} S_{1}-\left({ }^{4} S\right) 6 p{ }^{3} P_{1}$ \\
\hline 4748.95 & & 18 & $\left({ }^{2} P\right) S d \quad F_{3}-\left({ }^{2} P\right) 6 p \quad{ }^{\prime} D_{2}$ \\
\hline 4869.46 & & 21 & $\left({ }^{2} D\right) 5 d^{3} D_{2}-\left({ }^{2} D\right) 6 p{ }^{3} F_{3}$ \\
\hline 5238.93 & & 16 & $\left({ }^{2} D\right) S d{ }^{3} S_{1}-\left({ }^{4} S\right) 4 f^{5} F_{2}$ \\
\hline 5401.04 & & 17 & $\left({ }^{2} D\right) S d{ }^{3} P_{2}-\left({ }^{4} S\right) 4 f{ }^{3} F_{2}$ \\
\hline 5524.42 & \multirow[t]{6}{*}{$\mathrm{Xe}^{2+} ?$} & 8 & $\left.{ }^{(} P\right) 5 d{ }^{3} D_{2}-\left({ }^{2} D\right) 6 p{ }^{\prime} D_{2}$ \\
\hline 6176.6 & & & \\
\hline 6238.24 & & 13 & $\left({ }^{2} D\right) 5 d^{1} F_{3}-\left({ }^{2} D\right) 6 p{ }^{\prime} F_{3}$ \\
\hline 6343.5 & & & \\
\hline 7148.94 & & & \\
\hline 8571.6 & & & \\
\hline
\end{tabular}

- ? Ionisation stage of laser line uncertain.

b Rer. [27].

c Ref. [13] 